\title{
Homogeneous internal structure of CM-like asteroid (41) Daphne ${ }^{\star, \star \star}$
}

\author{
B. Carry ${ }^{1}$, F. Vachier ${ }^{2}$, J. Berthier ${ }^{2}$, M. Marsset ${ }^{3}$, P. Vernazza ${ }^{4}$, J. Grice ${ }^{1,5}$, W. J. Merline ${ }^{6}$, E. Lagadec ${ }^{1}$, A. Fienga ${ }^{7}$,
} A. Conrad ${ }^{8}$, E. Podlewska-Gaca ${ }^{9,11}$, T. Santana-Ros ${ }^{9}$, M. Viikinkoski ${ }^{12}$, J. Hanuš ${ }^{14}$, C. Dumas ${ }^{15}$, J. D. Drummond ${ }^{16}$, P. M. Tamblyn ${ }^{6,17}$, C. R. Chapman ${ }^{6}$, R. Behrend ${ }^{18}$, L. Bernasconi ${ }^{18}$, P. Bartczak ${ }^{9}$, Z. Benkhaldoun ${ }^{10}$, M. Birlan ${ }^{2}$, J. Castillo-Rogez ${ }^{19}$, F. Cipriani ${ }^{20}$, F. Colas ${ }^{2}$, A. Drouard ${ }^{4}$, J. Durech ${ }^{14}$, B. L. Enke ${ }^{6}$, S. Fauvaud ${ }^{18,21}$, M. Ferrais ${ }^{22}$, R. Fetick ${ }^{4}$, T. Fusco ${ }^{4}$, M. Gillon ${ }^{22}$, E. Jehin ${ }^{22}$, L. Jorda ${ }^{4}$, M. Kaasalainen ${ }^{12}$, M. Keppler ${ }^{13}$, A. Kryszczynska ${ }^{9}$,

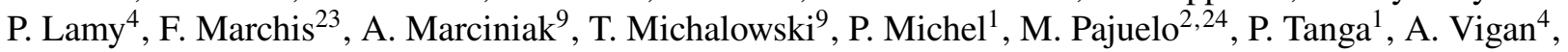
B. Warner ${ }^{25}$, O. Witasse ${ }^{20}$, B. Yang ${ }^{26}$, and A. Zurlo ${ }^{4,27,28}$

(Affiliations can be found after the references)

Received 18 July 2018 / Accepted 7 January 2019

\begin{abstract}
Context. CM-like asteroids (Ch and Cgh classes) are a major population within the broader C-complex, encompassing about $10 \%$ of the mass of the main asteroid belt. Their internal structure has been predicted to be homogeneous, based on their compositional similarity as inferred from spectroscopy and numerical modeling of their early thermal evolution.

Aims. Here we aim to test this hypothesis by deriving the density of the CM-like asteroid (41) Daphne from detailed modeling of its shape and the orbit of its small satellite.

Methods. We observed Daphne and its satellite within our imaging survey with the Very Large Telescope extreme adaptive-optics SPHERE/ZIMPOL camera and complemented this data set with earlier Keck/NIRC2 and VLT/NACO observations. We analyzed the dynamics of the satellite with our Genoid meta-heuristic algorithm. Combining our high-angular resolution images with optical lightcurves and stellar occultations, we determine the spin period, orientation, and 3D shape, using our ADAM shape modeling algorithm.

Results. The satellite orbits Daphne on an equatorial, quasi-circular, prograde orbit, like the satellites of many other large main-belt asteroids. The shape model of Daphne reveals several large flat areas that could be large impact craters. The mass determined from this orbit combined with the volume computed from the shape model implies a density for Daphne of $1.77 \pm 0.26 \mathrm{~g} \mathrm{~cm}^{-3}(3 \sigma)$. This density is consistent with a primordial CM-like homogeneous internal structure with some level of macroporosity $(\approx 17 \%)$.

Conclusions. Based on our analysis of the density of Daphne and 75 other $\mathrm{Ch} / \mathrm{Cgh}$-type asteroids gathered from the literature, we conclude that the primordial internal structure of the CM parent bodies was homogeneous.
\end{abstract}

Key words. minor planets, asteroids: general - minor planets, asteroids: individual: Daphne - methods: observational - techniques: high angular resolution

\section{Introduction}

The C-complex encompasses $50 \%$ of the mass of the asteroid belt (or $14 \%$ if the four largest bodies, Ceres, Vesta, Pallas and Hygeia, are disregarded, DeMeo \& Carry 2013, 2014). Within this complex, the Ch- and Cgh-types are defined by the presence of an absorption band around $0.7 \mu \mathrm{m}$, and a UV-dropoff (sharper

\footnotetext{
^ Based on observations made with (1) ESO Telescopes at the La Silla Paranal Observatory under programs 281.C-5011 (PI Dumas), 099.D-0098 (SPHERE GTO), and 199.C-0074(A) (PI Vernazza); and (2) the W. M. Keck Observatory, which is operated as a scientific partnership among the California Institute of Technology, the University of California and the National Aeronautics and Space Administration. The Observatory was made possible by the generous financial support of the W. M. Keck Foundation.

${ }^{\star \star}$ The reduced and deconvolved AO images and the 3D shape model are publicly available at http://observations.lam.fr/astero/ and at the CDS via anonymous ftp to cdsarc.u-strasbg.fr $(130.79 .128 .5)$ or via http://cdsarc.u-strasbg.fr/vizbin/qcat?J/A+A/623/A132
}

for the $\mathrm{Cgh}$ ). These have been estimated to represent between 30 and $65 \%$ by number (Rivkin 2012; Fornasier et al. 2014), and are associated with CM chondrites (namely the Ch- and Cgh-types, see Vilas et al. 1993; Burbine 1998; Bus \& Binzel 2002; DeMeo et al. 2009; Lantz et al. 2013; Fornasier et al. 2014; Vernazza et al. 2016; Takir et al. 2013). In other words, CM-like bodies represent a significant fraction of the C-complex population and encompass about $10 \%$ of the mass of all main-belt asteroids. They are spread over the entire Main Belt, and are found at all diameters (e.g., Rivkin 2012; Fornasier et al. 2014).

Their absorption band at $0.7 \mu \mathrm{m}$ has been associated with phyllosilicates (Vilas \& Sykes 1996). This is supported by the presence of a phyllosilicate band near $2.8 \mu \mathrm{m}$ (commonly called the $3 \mu \mathrm{m}$ absorption, Rivkin et al. 2015), as well as a shallow band at $2.33 \mu \mathrm{m}$. The $2.8 \mu \mathrm{m}$ band is very similar to that seen in the B-type asteroid (2) Pallas (Takir \& Emery 2012; Rivkin et al. 2015) and interpreted as being due to serpentine (e.g., Takir et al. 2013). The $2.33 \mu \mathrm{m}$ band is also associated with the serpentine group (i.e., hydrous magnesium-iron phyllosilicates, Beck et al. 2018). 
The meteorites originating from these bodies, the CM chondrites, represent $1.6 \%$ of all falls (Scott 2007). Together with CI carbonaceous chondrites, they represent the most chemically primitive meteorites (i.e., closest to the solar composition, Scott 2007) while paradoxically having suffered extensive hydration (e.g., Alexander et al. 2013). This aqueous alteration took place at low temperature, and thermal alteration of CM parent bodies peaked around $120^{\circ} \mathrm{C}$ (e.g., Dufresne \& Anders 1962; Zolensky et al. 1989, 1997; Guo \& Eiler 2007), and went up to $150^{\circ} \mathrm{C}$ as revealed by the formation of dolomite carbonates (Lee et al. 2014). As such, they are thought to have formed from a mixture of ice and dust where water ice was subsequently brought to a liquid state via the radioactive decay of ${ }^{26} \mathrm{Al}$ contained in dust particles, leading to the aqueous alteration of a significant fraction of the dust (see, e.g., Krot et al. 2006).

Recently, Vernazza et al. (2016) made a spectral survey of $70 \mathrm{Ch} / \mathrm{Cgh}$ asteroids, which included large 200+ km deemedprimordial bodies, but also objects as small as $15 \mathrm{~km}$ diameter, presumed to be the collisional fragments of dynamical families created from large bodies. This allowed a probe of the internal composition of prior, larger parent bodies. These authors interpreted the spectral diversity, already reported elsewhere (e.g., Vilas 1994; Fornasier et al. 1999, 2014), as resulting mainly from a variation of the average regolith grain size rather than to different thermal histories (Fornasier et al. 2014; Rivkin et al. 2015), although differences in band depths and centers also argue for some heterogeneity in mineral abundances (Burbine 1998; Cloutis et al. 2011; Fornasier et al. 2014). This evidence points toward an overall homogeneous internal structure for the parent bodies of CM chondrites.

The thermal modeling of the early internal structure of CM parent bodies by Bland \& Travis (2017) supports this conclusion. They showed that convection may have prevented strong thermal gradients and differentiation of material. Starting from the accretion of dust and ices beyond the snow line (Scott et al. 2018), the original non-lithified structure of CM parent bodies has allowed large-scale circulation of material. This model explains both the limited temperature experienced by $\mathrm{CM}$ parent bodies, and the small-scale heterogeneity (such as temperature and redox state observed at the hundred of micrometer level, see Fujiya et al. 2015) observed in CM chondrites (Guo \& Eiler 2007; Bland \& Travis 2017).

To test this model, we sought to gather evidence for or against an originally differentiated internal structure within large $\mathrm{Ch} / \mathrm{Cgh}$ asteroids. Our goal was to measure their bulk density, which, when compared with the density of CM chondrites, can reveal the presence, or absence, of denser material in their interior. Determination of a density requires both a mass and a volume. Masses for larger objects can be determined by their gravitational effects on other bodies, but the precision is often low, and is particularly problematic for objects of low mass (see Carry 2012, for a discussion on the precision and biases in mass determination). If any object is binary, however, the orbit of the satellite yields the primary mass directly. Therefore, binary asteroids are crucial to establish solid and accurate references on which a larger population can be analyzed.

The present article focuses on asteroid (41) Daphne as representative of the $\mathrm{Ch}$ spectral class, around which a satellite was discovered in 2008 (S/2008 (41) 1, which we call Peneius $^{1}$, Conrad et al. 2008). Daphne was observed within a survey we are currently conducting (ID 199.C-0074, PI P. Vernazza) to image a substantial fraction of main-belt asteroids

\footnotetext{
1 Pronounced "peh-NEH-oss."
}

larger than $100 \mathrm{~km}$ in diameter, sampling the main compositional classes (see Vernazza et al. 2018, for a description of the survey). We image these asteroids throughout their rotation at high angular-resolution with the SPHERE/ZIMPOL extreme adaptive-optics (AO) camera (Beuzit et al. 2008; Thalmann et al. 2008) mounted on the European Southern Observatory (ESO) Very Large Telescope (VLT). The high-quality correction delivered by the AO system of SPHERE (Fusco et al. 2006, 2014) compared to previous generations of AO cameras, and the use of shorter wavelength (visible $R$ band compared to the near-infrared $J / H / K$ bands typically used with previous cameras), provides a twofold to threefold improvement in angular resolution. This sharper resolution allows the detailed modeling of asteroid shapes and enhanced satellite detection capability, as recently illustrated on the main-belt asteroids (3) Juno, (6) Hebe, (16) Psyche, (89) Julia, (107) Camilla, and (130) Elektra (Viikinkoski et al. 2015, 2018; Marsset et al. 2016, 2017a,b; Yang et al. 2016; Hanuš et al. 2017a; Pajuelo et al. 2018; Vernazza et al. 2018).

The article is organized as follows: we first describe our observations in Sect. 2, followed by the determination of the 3D shape model (Sect. 3.1), the mutual orbit of Daphne and its satellite (Sect. 3.2), and the diameter and spectrum of the satellite itself (Sect. 3.3). Based on the density of (41) Daphne and a compilation of mass and diameter estimates from the literature, we then discuss the internal structure of CM parent bodies in Sect. 4.

\section{Observations}

Daphne was imaged with ZIMPOL once in May 2017 and three times in August 2018. We also compile 24 epochs of highangular resolution and high-contrast images (Table B.1) from large ground-based telescopes equipped with adaptive-optics (AO) cameras: NIRC2 at Keck (van Dam et al. 2004) and NACO at ESO VLT (Rousset et al. 2003).

We process the ZIMPOL data with the ESO pipeline (see details in Vernazza et al. 2018). We then reduce all other imaging epochs with the same suite of IDL routines for consistency. The basic data reduction encompasses bad pixel mapping and removal by median interpolation, sky subtraction, and flat-field correction, following the steps described in Carry et al. (2008). We then use Mistral, a myopic deconvolution algorithm optimized for celestial targets with sharp boundaries (Fusco et al. 2002; Mugnier et al. 2004), to deconvolve the images and enhance their angular resolution for shape modeling purposes. The results of this approach have already been demonstrated elsewhere (e.g., Witasse et al. 2006; Drummond et al. 2014). In parallel, the diffused halo of light surrounding Daphne was removed by subtracting concentric annuli (as described thoroughly in Pajuelo et al. 2018) for satellite detection.

We complement this data set with an additional epoch of Daphne in May 2017 with the SPHERE integral-field spectrograph (IFS, Claudi et al. 2008) to acquire the near-infrared spectrum of its satellite Peneius. This epoch was obtained within the "Other Science" program (ID: 099.D-0098, PI J.-L. Beuzit) of the guaranteed-time observations (GTO) of the SPHERE consortium. The aim of this program is to illustrate SPHERE's capabilities in science topics other than its primary goal: direct imaging of planets and disks. The IFS data were reduced using the SPHERE consortium pipeline, which includes bad pixel removal, sky subtraction, flat-field correction, and wavelength calibration. 
Together with these images, we retrieve nine stellar occultations by Daphne compiled on the PDS by Dunham et al. (2017). We convert the locations of observers and disappearance timings into chords on the plane of the sky using the recipes by Berthier (1999). We detail the circumstances of observation of these occultations in Table B.2.

We also compile 29 optical lightcurves, from the historical works of Scaltriti \& Zappala (1977), Barucci (1983), Barucci et al. (1985), and Weidenschilling et al. (1987, 1990), used by Kaasalainen et al. (2002) and Hanuš et al. (2017b) to reconstruct the 3D shape of Daphne. We complement this data set with twelve lightcurves obtained by amateur astronomers, two lightcurves obtained with the $60 \mathrm{~cm}$ André Peyrot telescope mounted at Les Makes observatory on Réunion Island (operated as a partnership among Les Makes Observatory and the IMCCE, Paris Observatory), one lightcurve obtained with the Antarctic Search for Transiting ExoPlanet (ASTEP) telescope (Daban et al. 2010) during its commissioning at the Observatoire de la Côte d'Azur in Nice (Matter et al. 2011), and extract three serendipitously observed lightcurves from the SuperWASP image archive (Grice et al. 2017). The details of these lightcurves are provided in Table B.3.

\section{Properties of Daphne and its satellite}

\subsection{Spin and $3 D$ shape}

We determine the spin properties (rotation period and spinvector coordinates) and reconstruct the $3 \mathrm{D}$ shape of Daphne with the open-source ${ }^{2}$ ADAM algorithm (Viikinkoski et al. 2015). ADAM uses the Levenberg-Marquardt optimization algorithm to find the spin and 3D shape that best reproduce the lightcurves, stellar occultation chords, and disk-resolved images simultaneously by comparing the observations with synthetic data generated by the model at each step.

The best-fit solution displayed in Fig. 1, the details of which are listed in Table 1, has a sidereal rotation period $P_{\mathrm{s}}$ of $5.98798 \mathrm{~h}$ and spin-vector ecliptic coordinates of $\left(199^{\circ},-33^{\circ}\right)$, very similar to the results from convex shape modeling from lightcurves only by Kaasalainen et al. (2002) and multi-data shape reconstruction from a slightly different data set with ADAM by Hanuš et al. (2017b). The shape model is of an irregular body with several large flat areas, putative impact basins, and has a spherical-volume-equivalent diameter $\mathcal{D}$ of $187 \pm 21.5 \mathrm{~km}$ ( $3 \sigma$ uncertainty). We present all the data compared with the predictions from the shape model in Appendix B.

An almost similar shape model and spin solution had been previously reported by Carry (2009) with the multi-data KOALA shape reconstruction algorithm (based on the same algorithm as ADAM but with different implementation, Carry et al. 2010a; Kaasalainen 2011). Because KOALA has been validated by comparing the shape model of (21) Lutetia (Carry et al. 2010b; Drummond et al. 2010) with the images returned by the ESA Rosetta mission during its flyby of the asteroid (Sierks et al. 2011; Carry et al. 2012), the agreement between the two models provides solid evidence for the reliability of both ADAM and of the shape model of Daphne.

While there is a significant spread of diameter estimates in the literature (average diameter of $192 \pm 38 \mathrm{~km}$ ), all the estimates based on direct measurements, i.e. stellar occultations, disk-resolved imaging, mid-infrared interferometry, are narrowly clustered around our value: $188 \pm 14 \mathrm{~km}$ ( $3 \sigma$ deviation, see

2 https://github.com/matvii/adam

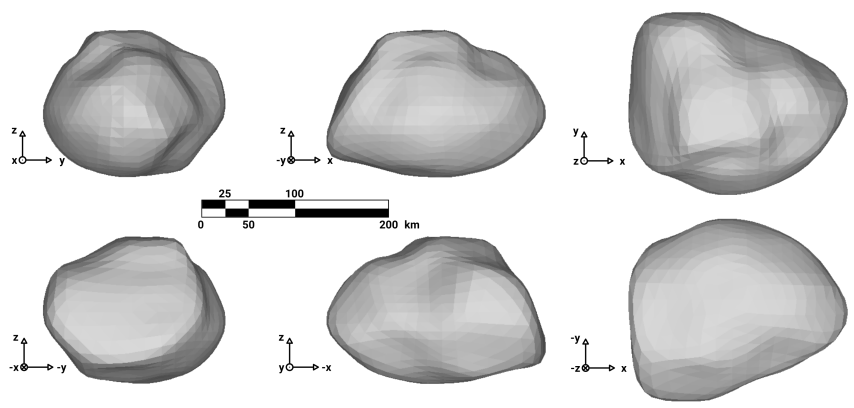

Fig. 1. Views of the shape model of Daphne. The $X, Y$ and $Z$ axes are aligned along the principal axes of inertia.

Table 1. Spin solution (coordinates in ecliptic and equatorial J2000 reference frames) and shape model parameters (the overall shape is reported as the $a>b>c$ diameters of a triaxial ellipsoid fit to the shape model).

\begin{tabular}{lllll}
\hline \hline Parameter & Symbol & Value & Unc. & Unit \\
\hline Sidereal period & $P_{\mathrm{s}}$ & 5.987981 & $6 \times 10^{-5}$ & $(\mathrm{~h})$ \\
Longitude & $\lambda$ & 199.4 & 5. & $\left(^{\circ}\right)$ \\
Latitude & $\beta$ & -31.9 & 5. & $\left(^{\circ}\right)$ \\
Right ascencion & $\alpha$ & 183.5 & 5. & $\left(^{\circ}\right)$ \\
Declination & $\delta$ & -36.6 & 5. & $\left(^{\circ}\right)$ \\
Ref. epoch & $T_{0}$ & 2444771.750 & & \\
\hline Diameter & $\mathcal{D}$ & 187 & 21.5 & $(\mathrm{~km})$ \\
Volume & $V$ & $3.39 \times 10^{6}$ & $7.1 \times 10^{5}$ & $(\mathrm{~km})$ \\
Diam. $a$ & $a$ & 237.9 & 21.5 & $(\mathrm{~km})$ \\
Diam. $b$ & $b$ & 184.8 & 21.5 & $(\mathrm{~km})$ \\
Diam. $c$ & $c$ & 156.0 & 21.5 & $(\mathrm{~km})$ \\
Axes ratio & $a / b$ & 1.29 & 0.19 & \\
Axes ratio & $b / c$ & 1.18 & 0.21 & \\
Axes ratio & $a / c$ & 1.52 & 0.25 & \\
\hline
\end{tabular}

Notes. All uncertainties are reported at $3 \sigma$.

Table A.1). In particular, the mid-infrared interferometric observations by Matter et al. (2011) provide an independent confirmation, being based on a totally different data set. These authors analyzed their interferometric visibilities using the convex shape model of Kaasalainen et al. (2002) and the non-convex shape model of Carry (2009), almost identical to the model presented here. The associated diameters differ by $15 \mathrm{~km}$, showing how the determination of diameter is sensitive to the shape of the object. Their best-fit diameter associated with the non-convex model was $185.5 \pm 10.5 \mathrm{~km}(3 \sigma)$, supporting the present value.

We define the prime meridian of Daphne to be along its longest axis, on which a hill, which we call the nose, is present at the equator (elevation of $11 \mathrm{~km}$ above the reference ellipsoid, see the map in Fig. 2). Several flat regions (hereafter A, B, C) can be identified in the images and on the shape model, as well as a clear depression (D). The three flat areas are located near both poles, and on the opposite side of the nose. These flat areas and the depression are large compared with the diameter of Daphne and may be indicative of large impact basins not modeled because concavities could not be detected with the available data (Ďrech \& Kaasalainen 2003; Devogèle et al. 2015). The central location and overall dimensions of these features, including the ratio of their surface-equivalent diameter to the diameter of Daphne, are listed in Table 2. 


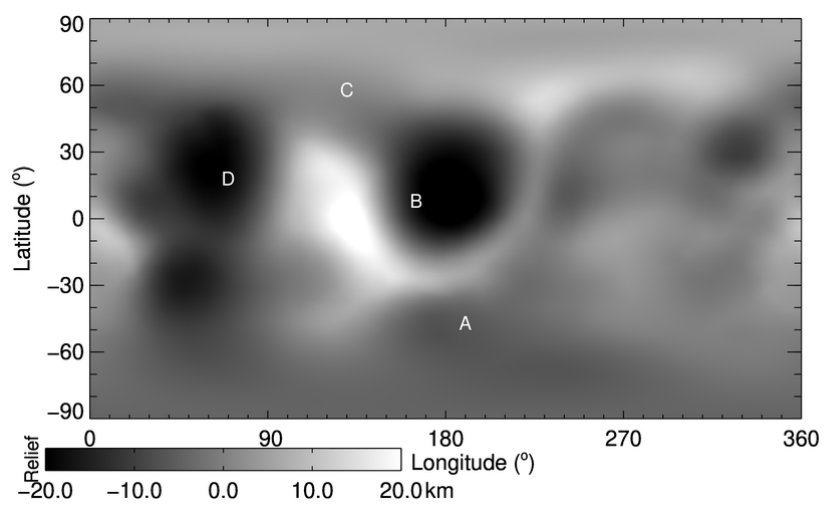

Fig. 2. Topography of Daphne, measured in kilometers with respect to its reference ellipsoid (Table 1). The putative depression is marked by the letter D, while the three flat areas are labeled A, B, and C. They may appear as circular depressions here (in particular B) because the topography is measured with respect to an ellipsoid.

Table 2. Dimensions (semi-axes $\mathcal{L}_{a}$ and $\mathcal{L}_{b}$, surface area $\mathcal{A}$, and fraction $f$ of Daphne's diameter) and planetocentric coordinates $\left(\lambda_{c}, \beta_{c}\right)$ of the four notable topographic features of Daphne.

\begin{tabular}{lccccl}
\hline \hline & $\mathrm{A}$ & $\mathrm{B}$ & $\mathrm{C}$ & $\mathrm{D}$ & Unit \\
\hline$\lambda_{c}$ & $190 \pm 5$ & $165 \pm 5$ & $130 \pm 5$ & $60 \pm 5$ & $\left({ }^{\circ}\right)$ \\
$\beta_{c}$ & $-50 \pm 5$ & $+5 \pm 5$ & $+55 \pm 5$ & $+19 \pm 5$ & $\left({ }^{\circ}\right)$ \\
$\mathcal{L}_{a}$ & $60 \pm 5$ & $68 \pm 5$ & $45 \pm 5$ & $42 \pm 3$ & $(\mathrm{~km})$ \\
$\mathcal{L}_{b}$ & $48 \pm 5$ & $42 \pm 5$ & $45 \pm 5$ & $42 \pm 3$ & $(\mathrm{~km})$ \\
$\mathcal{A}$ & $8.7 \pm 3.3$ & $9.0 \pm 3.3$ & $6.3 \pm 2.8$ & $5.5 \pm 0.8$ & $\left(10^{3} \mathrm{~km}^{2}\right)$ \\
$f$ & $56 \pm 10$ & $57 \pm 10$ & $47 \pm 10$ & $44 \pm 3$ & $(\%)$ \\
\hline
\end{tabular}

\subsection{Dynamics of the system}

On each image, we measure the relative position of the satellite with respect to center of light of the primary by adjusting a $2 \mathrm{D}$ gaussian on the primary using the unmodified images and another on the satellite using the halo-removed images (Fig. 3). We use the meta-heuristic algorithm Genoid (Vachier et al. 2012) to find the set of orbital parameters that best fit the observations. The orbital parameter space is usually $6 \mathrm{D}$ for a simple Keplerian motion: orbital period, excentricity, inclination, longitude of the ascending node, argument of periapsis, and time of passage to the periapsis. More dimensions are required if the gravitational potential is not central, such as in accounting for a gravitational quadrupole $\mathbf{J}_{2}$. Genoid explores this parameter space in successive generations of orbital solutions, randomly merging the parameters of the best solutions to create newer generations. In combination with this broad exploration of the parameter space, Genoid uses gradient descent at each generation to find the minimum closest to each best-trial solution.

The reliability of this approach has been assessed during a stellar occultation by (87) Sylvia in 2013. We had used Genoid to predict the position of its largest satellite Romulus before the event, placing observers on the occultation path of the satellite. Four different observers detected an occultation by Romulus at only $13.5 \mathrm{~km}$ off the predicted position (Berthier et al. 2014).

The best-fit orbit adjusts the 30 positions with a root-mean square (rms) residual of 8.3 mas only, i.e., smaller than the pixel size of most observations (21 out of 30 taken with NACO/VLT
Table 3. Orbital elements of Peneius, the satellite of Daphne, expressed in EQJ2000, obtained with Genoid.

\begin{tabular}{lll}
\hline \hline \multicolumn{3}{c}{ Observing data set } \\
Number of observations & 30 \\
Time span (days) & 3783 & \\
rms (mas) & 8.3 & \\
\hline \multicolumn{3}{c}{ Orbital elements EQJ2000 } \\
$P($ day) & 1.137446 & \pm 0.000009 \\
$a(\mathrm{~km})$ & 463.5 & \pm 22.8 \\
$e$ & 0.009 & +0.021 \\
$i\left(^{\circ}\right)$ & 128.1 & -0.009 \\
$\Omega\left({ }^{\circ}\right)$ & \pm 6.2 \\
$\omega\left(^{\circ}\right)$ & 272.7 & \pm 5.7 \\
$t_{\mathrm{p}}(\mathrm{JD})$ & 171.9 & \pm 37.6 \\
\hline \multicolumn{3}{c}{ Derived parameters } \\
$M\left(\times 10^{18} \mathrm{~kg}\right)$ & 2454550.586985 & \pm 0.117331 \\
$\lambda_{p}, \beta_{p}\left({ }^{\circ}\right)$ & 6.10 & \\
$\alpha_{p}, \delta_{p}\left({ }^{\circ}\right)$ & $197,-33$ & \pm 0.89 \\
$\Lambda\left(^{\circ}\right)$ & $182,-38$ & $\pm 6,7$ \\
\hline
\end{tabular}

Notes. The table lists: orbital period $P$, semi-major axis $a$, eccentricity $e$, inclination $i$, longitude of the ascending node $\Omega$, argument of pericenter $\omega$, time of pericenter $t_{\mathrm{p}}$. The number of observations and rms between predicted and observed positions are also provided. Finally, we report the derived primary mass $M$, the ecliptic J2000 coordinates of the orbital pole $\left(\lambda_{p}, \beta_{p}\right)$, the equatorial $\mathrm{J} 2000$ coordinates of the orbital pole $\left(\alpha_{p}, \delta_{p}\right)$, and the orbital inclination $(\Lambda)$ with respect to the equator of Daphne. Uncertainties are given at $3 \sigma$.

and NIRC2/Keck, see Table C.1). The observations, covering 3783 days or 3326 revolutions, provide solid constraints on the orbital period $(1.137446 \pm 0.000009$ day). The satellite orbits Daphne on a Keplerian, equatorial, and prograde orbit, slightly eccentric (Table 3 ), at a distance of only 7.4 primary radii. We searched for a signature of the gravitational quadrupole $\mathbf{J}_{2}$ but the poor time coverage of the astrometry with a gap of nine years between the positions in 2008 and those in 2017 and 2018 precluded any firm conclusion. A regular follow-up of the position of Peneius is required to conclude on the $J_{2}$ of Daphne. These orbital characteristics argue in favor of a formation of the satellite by impact excavation, and re-accumulation of material in orbit, followed by tidal circularization (Weidenschilling et al. 1989; Merline et al. 2002; Durda et al. 2004; Margot et al. 2015).

\subsection{Spectrum and diameter of the satellite}

We recorded the near-infrared spectra of Daphne and its satellite with SPHERE/IFS. Telluric features were removed by observing the nearby star HD 102085 (G3V). Similarly to previous sections, the bright halo of Daphne that contaminated the spectrum of the moon was removed. This was achieved by measuring the background at the location of the moon for each pixel as the median value of the area defined as a $40 \times 1$-pixel arc centered on Daphne. To estimate the uncertainty and potential bias on photometry (at each wavelength) introduced by this method, we performed a number of simulations (similarly to our study of the satellite of Camilla, see Pajuelo et al. 2018).

We added synthetic companions on the 39 spectral images of the spectro-imaging cube, at a similar separation to the satellite $(\approx 300$ mas) and random position angles from the primary. The simulated sources were modeled as the point-spread function 

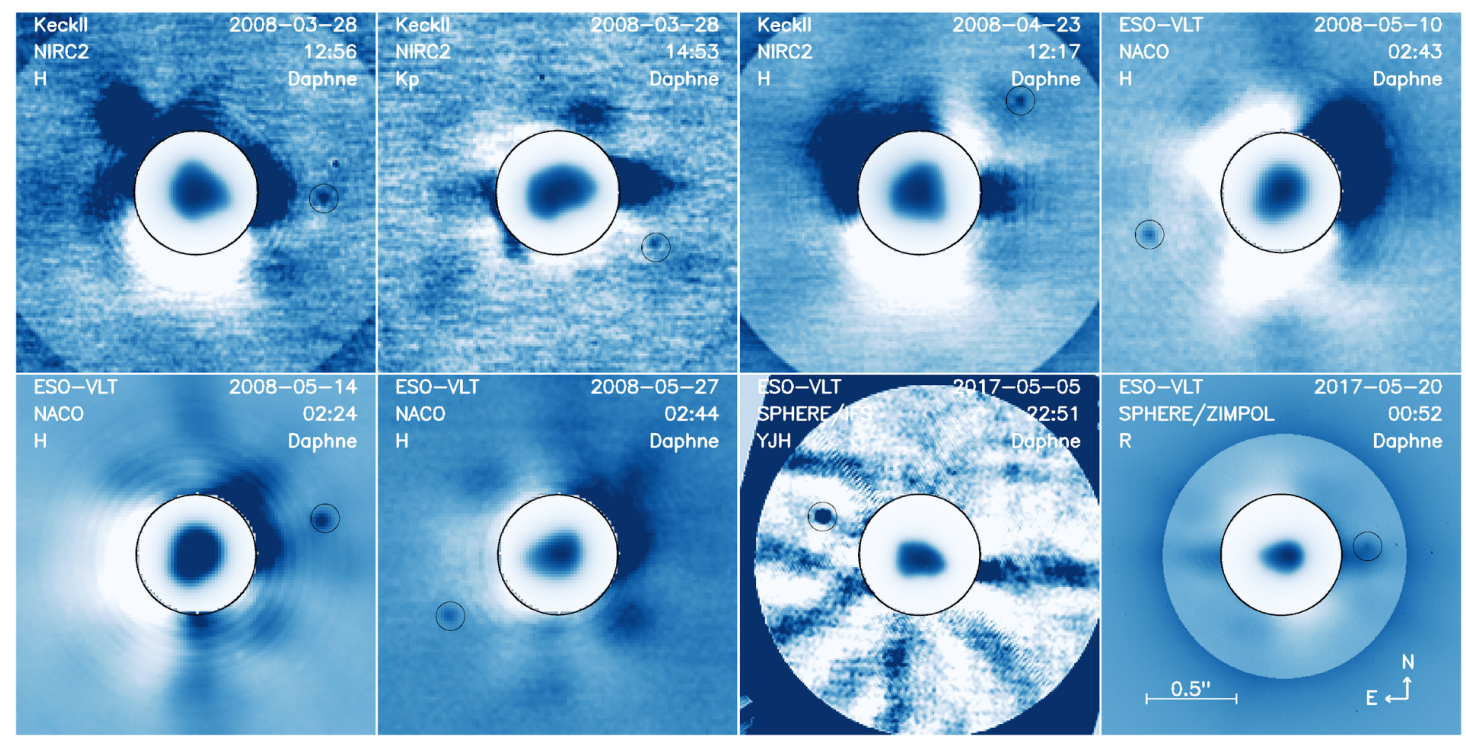

Fig. 3. Example images of Daphne and its satellite. Each panel represents a different epoch. The image is displayed in the inner circle, showing the irregular shape of Daphne. The outer region shows the images after halo removal, showing the satellite (highlighted by a small circle). On each panel the telescope, instrument, filter, and UTC date are reported.

from the calibration star images scaled in brightness. The halo from Daphne was then removed from these simulated images using the method described above, and the flux of the simulated companion measured by adjusting a 2D-Gaussian profile (see Sect. 3.2). Based on a total statistics of 100 simulated companions, we find that the median loss of flux at each wavelength is $13 \pm 9 \%$ and that the spectral slope is affected $(-0.50 \% / 100 \mathrm{~nm}$ on average).

The measured slope for each individual simulation, however, is not reliable, the standard deviation of all simulations being of $2.73 \% / 100 \mathrm{~nm}$. The value for each depends on the location (position angle) of the simulated satellite. Furthermore, the signal-to-noise ratio $(\mathrm{S} / \mathrm{N})$ of the simulations range from virtually zero to four, with an average of 1.8 only. The spectrum of the satellite, therefore, is not reliable, being noisy and likely affected by strong slope effects.

From the integrated fluxes measured with the 2D gaussian functions on Daphne and its satellite at each epoch (Fig. C.2 and Table C.1), we derived their magnitude difference to be $9.49 \pm 0.32$. Combined with our determination of a diameter of $187 \pm 21.5 \mathrm{~km}$, we derive a diameter of $2.4_{-1.1}^{+1.8} \mathrm{~km}$ for the satellite, under the assumption of a similar albedo for both. This assumption is supported by the multiple reports of spectral similarities between the components of multiple asteroid systems: (22) Kalliope (Laver et al. 2009), (90) Antiope (Polishook et al. 2009), (107) Camilla (Pajuelo et al. 2018), (130) Elektra (Yang et al. 2016), and (379) Huenna (DeMeo et al. 2011).

\section{Implications on the internal structure}

Using the volume determined from ADAM (Sect. 3.1) and the mass from Genoid (Sect. 3.2), we derive a bulk density of $1.77 \pm 0.26$ ( $\mathrm{g} \mathrm{cm}^{-3}, 3 \sigma$ uncertainty). This value is smaller but marginally consistent with the typical density of the CM carbonaceous chondrites, at $2.13 \pm 0.57 \mathrm{~g} \mathrm{~cm}^{-3}$ ( $3 \sigma$, Consolmagno et al. 2008). Comparing these two density values implies a macroporosity of $17 \pm 3 \%$.

As visible in Fig. 4, Ch and Cgh asteroids over two orders of magnitude in mass follow the same trend and have a density smaller than that of CM meteorites, although there is definitively some scatter due to large uncertainties and biases in the determination of their density (see Appendix D for the complete list of estimates, and Carry 2012, for a discussion on the reliability of mass, diameter, and density estimates). The distribution of all density estimates weighted by their respective uncertainty is Gaussian with an average value of $1.40_{-1.40}^{+1.92} \mathrm{~g} \mathrm{~cm}^{-3}$. This value changes to $1.58 \pm 0.97 \mathrm{~g} \mathrm{~cm}^{-3}(3 \sigma)$ by considering only the estimates with less than $20 \%$ relative uncertainty (represented by the blue curves in Fig. 4). This highlights the importance of the study of binary systems that can be angularly resolved to accurately determine both the volume and the mass, hence the density.

There are three large $(150+\mathrm{km})$ binary Ch-type asteroids: (41) Daphne, (121) Hermione, and (130) Elektra. The density of Hermione was estimated to $1.4_{-0.6}^{+1.5} \mathrm{~g} \mathrm{~cm}^{-3}$ by Descamps et al. (2009) and $1.26 \pm 0.90 \mathrm{~g} \mathrm{~cm}^{-3}$ by Viikinkoski et al. (2017). The density of Elektra was reported as $1.60 \pm 0.39 \mathrm{~g} \mathrm{~cm}^{-3}$ by Hanuš et al. (2017a), based on the mass estimate by Marchis et al. (2008a) while the mass recently derived by Yang et al. (2016) leads to $1.50 \pm 0.36 \mathrm{~g} \mathrm{~cm}^{-3}$. These values are somewhat smaller than the density of the three other large Ch-types: (13) Egeria at $1.99 \pm 0.69 \mathrm{~g} \mathrm{~cm}^{-3}$, (19) Fortuna at $1.96 \pm 0.28 \mathrm{~g} \mathrm{~cm}^{-3}$, and (48) Doris at $1.63 \pm 0.74 \mathrm{~g} \mathrm{~cm}^{-3}$ (see Appendix D). The density of these six targets is systematically smaller than $\mathrm{CM}$ meteorites.

Over the wide range of mass spanned by the $\mathrm{Ch} / \mathrm{Cgh}$ asteroids studied here (Fig. 4), the density appears roughly constant although there is a larger spread toward smaller diameters. This larger spread is likely due to larger uncertainties on the density estimates (the gravitational signature of asteroids become harder to detect toward smaller sizes, see Carry 2012). Indeed, the report by Carry (2012) of a correlation between density and mass (or diameter) due to an increasing macroporosity toward smaller diameters mainly happens for objects smaller than $100 \mathrm{~km}$. This density of $\mathrm{Ch} / \mathrm{Cgh}$ asteroids systematically lower than that of $\mathrm{CM}$ meteorites has two implications.

First, the density of the largest $\mathrm{Ch} / \mathrm{Cgh}$ asteroids argues against the presence of material denser than CM-like material in their interior, i.e., differentiation. If denser material was present, their macroporosity would have to be large to maintain their bulk 


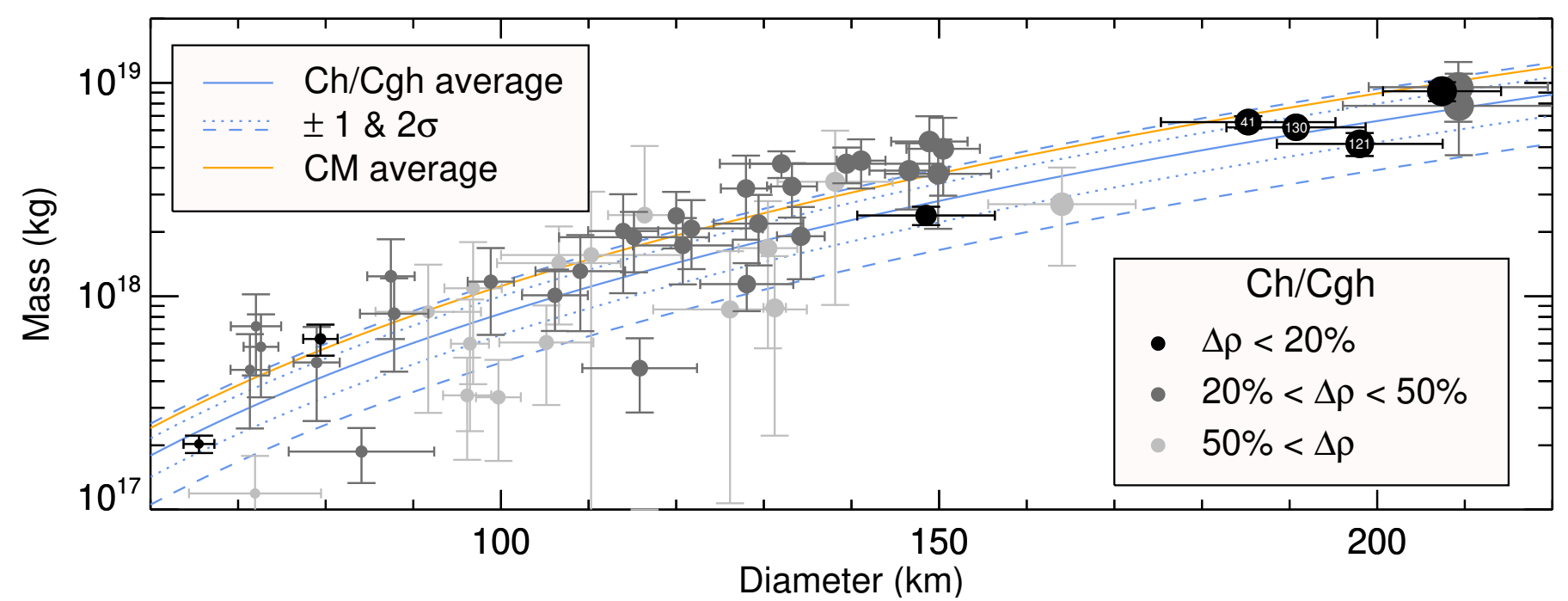

Fig. 4. Mass vs. diameter distribution for $76 \mathrm{Ch} / \mathrm{Cgh}$ asteroids (grey circles). Those more precise than 50 and $20 \%$ are plotted as dark grey and black circles, respectively. Unreliable density estimates (below $0.5 \mathrm{~g} \mathrm{~cm}^{-3}$ ) are not displayed. The three binary asteroids, (41) Daphne, (121) Hermione, (130) Elektra, are labeled. The solid and dashed blue curves represent the average density $\left(1.58 \mathrm{~g} \mathrm{~cm}^{-3}\right)$ and $1 \sigma-2 \sigma\left( \pm 0.33 \mathrm{~g} \mathrm{~cm}^{-3}\right)$ dispersion of the sample. The solid orange curve stands for the average density of CM chondrites at $2.13 \pm 0.57 \mathrm{~g} \mathrm{~cm}^{-3}(3 \sigma)$.

density, contrarily to what is observed for 100-200+ km bodies (Carry 2012). Together with the observed compositional homogeneity of $\mathrm{Ch} / \mathrm{Cgh}$ asteroids (Vernazza et al. 2016), it supports the idea of an originally undeifferentiated internal structure of $\mathrm{CM}$ parent bodies. Because collisional fragments sample the interior of their parent bodies, this spectral homogeneity only modulated by grain sizes together with the density smaller than the surface analog material can be interpreted as evidence for a homogeneous internal structure, without differentiation, of the CM parent bodies. The thermal modeling in "giant mud balls" (non-lithified structure) proposed by Bland \& Travis (2017) then provides an explanation for the observed peak temperature of $120^{\circ} \mathrm{C}$ (e.g., Guo \& Eiler 2007).

Second, the density of $\mathrm{Ch} / \mathrm{Cgh}$ asteroids being systematically smaller than CM chondrite indicates the presence of large voids, or less dense material, in their interior. Given the extensive hydration suffered by $\mathrm{CM}$ chondrites (e.g., Alexander et al. 2013) and the detection of water ice on/near the surface of asteroids well-within the snowline (e.g., Campins et al. 2010; Küppers et al. 2014), one cannot reject the hypothesis of the presence of water ice in $\mathrm{Ch} / \mathrm{Cgh}$ asteroids. However, considering that all these objects have experienced numerous collisions over the history of the solar system, some macroporosity, i.e. voids, can be expected.

\section{Conclusion}

We have acquired high-angular resolution images and spectroimages of the binary Ch-type asteroid (41) Daphne from large ground-based telescopes, including the new generation extreme adaptive-optics SPHERE camera mounted on the ESO VLT. We determined the orbit of its small satellite Peneius from 30 observations of its position around Daphne, with an rms of 8.3 mas only. Combining our disk-resolved images with optical lightcurves and stellar occultations, we determined the 3D shape of (41) Daphne.

The derived density of $1.77 \pm 0.26 \mathrm{~g} \mathrm{~cm}^{-3}$ provides a solid reference to interpret the density of $\mathrm{Ch} / \mathrm{Cgh}$ asteroids. The density is available for 76 of them and is found systematically smaller than that of the associated CM carbonaceous chondrites $\left(2.13 \pm 0.57 \mathrm{~g} \mathrm{~cm}^{-3}, 3 \sigma\right)$, including for the largest asteroids. This provides robust evidence for a primordial homogeneous internal structure of these asteroids, in agreement with the observation of compositionnal homogeneity among $\mathrm{Ch} / \mathrm{Cgh}$ asteroids of very different diameters by Vernazza et al. (2016) and the modeling of their early thermal history by Bland \& Travis (2017).

Acknowledgements. Some of the work presented here is based on observations collected at the European Organisation for Astronomical Research in the Southern Hemisphere under ESO programs 281.C-5011 (PI Dumas), 099.D-0098, (SPHERE GTO), and 199.C-0074(A) (PI Vernazza). Some of the data presented herein were obtained at the W. M. Keck Observatory, which is operated as a scientific partnership among the California Institute of Technology, the University of California and the National Aeronautics and Space Administration. The Observatory was made possible by the generous financial support of the W. M. Keck Foundation. This research has made use of the Keck Observatory Archive (KOA), which is operated by the W. M. Keck Observatory and the NASA Exoplanet Science Institute (NExScI), under contract with the National Aeronautics and Space Administration. We thank the AGORA association which administrates the $60 \mathrm{~cm}$ telescope at Les Makes observatory, under a financial agreement with Paris Observatory. Thanks to A. Peyrot, J.-P. Teng for local support, and A. Klotz for helping with the robotizing. Some of these observations were acquired under grants from the National Science Foundation and NASA to W.J.M. (PI). B.C., A.D., J.G. and P.V. were supported by CNRS/INSU/PNP. J.H. and J.D. were supported by the grant 18-09470S of the Czech Science Foundation. The research leading to these results has received funding from the European Union's Horizon 2020 Research and Innovation Programme, under Grant Agreement no 687378. This paper makes use of data from the DR1 of the WASP data (Butters et al. 2010) as provided by the WASP consortium, and the computing and storage facilities at the CERIT Scientific Cloud, reg. no. CZ.1.05/3.2.00/08.0144 which is operated by Masaryk University, Czech Republic. TRAPPIST-South is funded by the Belgian Fund for Scientific Research (Fond National de la Recherche Scientifique, FNRS) under the grant FRFC 2.5.594.09.F, with the participation of the Swiss FNS. TRAPPIST-North is a project funded by the University of Liège, and performed in collaboration with Cadi Ayyad University of Marrakesh. E.J. and M.G. are Belgian FNRS Senior Research Associates. The authors wish to recognize and acknowledge the very significant cultural role and reverence that the summit of Mauna Kea has always had within the indigenous Hawaiian community. We are most fortunate to have the opportunity to conduct observations from this mountain. Thanks to all the amateurs worldwide who regularly observe asteroid lightcurves and stellar occultations. Some co-authors of this study are amateurs who observed Daphne, and provided crucial data. The authors acknowledge the use of the Virtual Observatory tools Miriade ${ }^{3}$ (Berthier et al. 2008),

3 Miriade: http://vo.imcce.fr/webservices/miriade/ 
B. Carry et al.: Homogeneous internal structure of CM-like asteroid (41) Daphne

TOPCAT $^{4}$, and STILTS ${ }^{5}$ (Taylor 2005). This research used the SSOIS ${ }^{6}$ facility of the Canadian Astronomy Data Centre operated by the National Research Council of Canada with the support of the Canadian Space Agency (Gwyn et al. 2012). Part of the data utilized in this publication were obtained and made available by the MIT-UH-IRTF Joint Campaign for NEO Reconnaissance, using SpeX spectrograph (Rayner et al. 2003). The IRTF is operated by the University of Hawaii under Cooperative Agreement no. NCC 5-538 with the National Aeronautics and Space Administration, Office of Space Science, Planetary Astronomy Program. The MIT component of this work is supported by NASA grant 09-NEOO009-0001, and by the National Science Foundation under Grant Nos. 0506716 and 0907766. We wish to acknowledge Professor Robert Groves, Director of Basic Languages (Classics) of the University of Arizona, for his assistance in selecting the name for Daphne's satellite.

\section{References}

Alexander, C. M. O., Howard, K. T., Bowden, R., \& Fogel, M. L. 2013, Geochim. Cosmochim. Acta, 123, 244

Baer, J., \& Chesley, S. R. 2017, AJ, 154, 76

Baer, J., Milani, A., Chesley, S. R., \& Matson, R. D. 2008, BAAS, 40, 493

Baer, J., Chesley, S. R., \& Matson, R. D. 2011, AJ, 141, 143

Barucci, M. A. 1983, A\&AS, 54, 471

Barucci, M. A., Fulchignoni, M., Burchi, R., \& D’Ambrosio, V. 1985, Icarus, 61 152

Beck, P., Maturilli, A., Garenne, A., et al. 2018, Icarus, 313, 124

Berthier, J. 1999, Notes scientifique et techniques du Bureau des longitudes (Paris: Bureau des Longitudes), S064

Berthier, J., Hestroffer, D., Carry, B., et al. 2008, LPI Contributions, 1405, 8374

Berthier, J., Vachier, F., Marchis, F., Durech, J., \& Carry, B. 2014, Icarus, 239, 118

Beuzit, J.-L., Feldt, M., Dohlen, K., et al. 2008, in Ground-based and Airborne Instrumentation for Astronomy II, Proc. SPIE, 7014, 701418

Bland, P. A., \& Travis, B. J. 2017, Sci. Adv., 3, e1602514

Burbine, T. H. 1998, Meteorit. Planet. Sci., 33, 253

Bus, S. J., \& Binzel, R. P. 2002, Icarus, 158, 146

Butters, O. W., West, R. G., Anderson, D. R., et al. 2010, A\&A, 520, L10

Campins, H., Hargrove, K., Pinilla-Alonso, N., et al. 2010, Nature, 464, 1320

Carry, B. 2009, Ph.D. Thesis, Observatoire de Paris, Paris

Carry, B. 2012, Planet. Space Sci., 73, 98

Carry, B., Dumas, C., Fulchignoni, M., et al. 2008, A\&A, 478, 235

Carry, B., Dumas, C., Kaasalainen, M., et al. 2010a, Icarus, 205, 460

Carry, B., Kaasalainen, M., Leyrat, C., et al. 2010b, A\&A, 523, A94

Carry, B., Kaasalainen, M., Merline, W. J., et al. 2012, Planet. Space Sci., 66, 200

Claudi, R. U., Turatto, M., Gratton, R. G., et al. 2008, in Ground-based and Airborne Instrumentation for Astronomy II, Proc. SPIE, 7014, 70143E

Cloutis, E. A., Hudon, P., Hiroi, T., Gaffey, M. J., \& Mann, P. 2011, Icarus, 216, 309

Conrad, A. R., Merline, W. J., Drummond, J. D., et al. 2008, IAU Circ., 8930, 2

Consolmagno, G., Britt, D., \& Macke, R. 2008, Chemie der Erde/Geochemistry, 68,1

Daban, J.-B., Gouvret, C., Guillot, T., et al. 2010, Proc. SPIE, 7733, 77334T

DeMeo, F., \& Carry, B. 2013, Icarus, 226, 723

DeMeo, F., \& Carry, B. 2014, Nature, 505, 629

DeMeo, F., Binzel, R. P., Slivan, S. M., \& Bus, S. J. 2009, Icarus, 202, 160

DeMeo, F. E., Carry, B., Marchis, F., et al. 2011, Icarus, 212, 677

Descamps, P., Marchis, F., Durech, J., et al. 2009, Icarus, 203, 88

Devogèle, M., Rivet, J. P., Tanga, P., et al. 2015, MNRAS, 453, 2232

Drummond, J. D., Conrad, A., Merline, W. J., et al. 2010, A\&A, 523, A93

Drummond, J. D., Merline, W. J., Conrad, A., et al. 2011, AAS/Division for Planetary Sciences Meeting Abstracts, 43, 41

Drummond, J. D., Carry, B., Merline, W. J., et al. 2014, Icarus, 236, 28

Dufresne, E. R., \& Anders, E. 1962, Geochim. Cosmochim. Acta, 26, 1085

Dunham, D. W., Herald, D., Frappa, E., et al. 2017, Asteroid Occultations, NASA Planetary Data System, EAR-A-3-RDR-OCCULTATIONS-V15.0

Durda, D. D., Bottke, W. F., Enke, B. L., et al. 2004, Icarus, 170, 243

Durech, J., \& Kaasalainen, M. 2003, A\&A, 404, 709

Durech, J., Kaasalainen, M., Herald, D., et al. 2011, Icarus, 214, 652

Fienga, A., Laskar, J., Morley, T., et al. 2009, A\&A, 507, 1675

Fienga, A., Kuchynka, P., Laskar, J., Manche, H., \& Gastineau, M. 2011, EPSC-DPS Joint Meeting 2011, 1879

4 TOPCAT: http://www.star.bris.ac.uk/ mbt/topcat/

5 STILTS: http://wwW.star.bris.ac.uk/ mbt/stilts/

6 SSOIS: http://www.cadc-ccda.hia-iha.nrc-cnrc.gc.ca/en/ ssois
Fienga, A., Manche, H., Laskar, J., Gastineau, M., \& Verma, A. 2013, ArXiv e-prints [arXiv: 1301.1510$]$

Fienga, A., Manche, H., Laskar, J., Gastineau, M., \& Verma, A. 2014, Scientific notes, https://www . imcce.fr/recherche/equipes/asd/inpop/ download13c

Folkner, W. M., Williams, J. G., \& Boggs, D. H. 2009, IPN Progress Report, 42, 1

Fornasier, S., Lazzarin, M., Barbieri, C., \& Barucci, M. A. 1999, A\&AS, 135, 65 Fornasier, S., Lantz, C., Barucci, M. A., \& Lazzarin, M. 2014, Icarus, 233, 163

Fujiya, W., Sugiura, N., Marrocchi, Y., et al. 2015, Geochim. Cosmochim. Acta, 161,101

Fusco, T., Conan, J.-M., Michau, V., \& Rousset, G. 2002, in Optics in Atmospheric Propagation and Adaptive Systems IV, eds. A. Kohnle, J. D. Gonglewski \& T. J. Schmugge, Proc. SPIE, 4538, 144

Fusco, T., Rousset, G., Sauvage, J.-F., et al. 2006, Opt. Exp., 14, 7515

Fusco, T., Sauvage, J.-F., Petit, C., et al. 2014, in Adaptive Optics Systems IV, Proc. SPIE, 9148, 91481U

Goffin, E. 2014, A\&A, 565, A56

Grav, T., Mainzer, A. K., Bauer, J., et al. 2012, ApJ, 744, 197

Grice, J., Snodgrass, C., Green, S., Parley, N., \& Carry, B. 2017, Asteroids, Comets, and Meteors: ACM 2017

Guo, W., \& Eiler, J. M. 2007, Geochim. Cosmochim. Acta, 71, 5565

Gwyn, S. D. J., Hill, N., \& Kavelaars, J. J. 2012, PASP, 124, 579

Hanuš, J., Durech, J., Brož, M., et al. 2013a, A\&A, 551, A67

Hanuš, J., Marchis, F., \& Durech, J. 2013b, Icarus, 226, 1045

Hanuš, J., Marchis, F., Viikinkoski, M., Yang, B., \& Kaasalainen, M. 2017a, A\&A, 599, A36

Hanuš, J., Viikinkoski, M., Marchis, F., et al. 2017b, A\&A, 601, A114

Hasegawa, S., Müller, T. G., Kuroda, D., Takita, S., \& Usui, F. 2013, PASJ, 65, 34

Ivantsov, A. 2008, Planet. Space Sci., 56, 1857

Kaasalainen, M. 2011, AIMS, 5, 37

Kaasalainen, M., Torppa, J., \& Piironen, J. 2002, A\&A, 383, L19

Kochetova, O. M. 2004, Solar Syst. Res., 38, 66

Kochetova, O. M., \& Chernetenko, Y. A. 2014, Solar Syst. Res., 48, 295

Konopliv, A. S., Asmar, S. W., Folkner, W. M., et al. 2011, Icarus, 211, 401

Krasinsky, G. A., Pitjeva, E. V., Vasiliev, M. V., \& Yagudina, E. I. 2001, in Communications of IAA of RAS

Kretlow, M. 2014, Minor Planet Bull., 41, 194

Krot, A. N., Hutcheon, I. D., Brearley, A. J., et al. 2006, Meteorites and the Early Solar System II, eds. D. S. Lauretta \& H. Y. McSween (Tucson, AZ: University of Arizona Press), 525

Kuchynka, P., \& Folkner, W. M. 2013, Icarus, 222, 243

Küppers, M., O’Rourke, L., Bockelée-Morvan, D., et al. 2014, Nature, 505, 525

Lantz, C., Clark, B. E., Barucci, M. A., \& Lauretta, D. S. 2013, A\&A, 554, A138

Laver, C., de Pater, I., Marchis, F., Ádámkovics, M., \& Wong, M. H. 2009, Icarus, 204, 574

Lee, M. R., Lindgren, P., \& Sofe, M. R. 2014, Geochim. Cosmochim. Acta, 144 126

Marchis, F., Hestroffer, D., Descamps, P., et al. 2005, Icarus, 178, 450

Marchis, F., Kaasalainen, M., Hom, E. F. Y., et al. 2006, Icarus, 185, 39

Marchis, F., Descamps, P., Baek, M., et al. 2008a, Icarus, 196, 97

Marchis, F., Descamps, P., Berthier, J., et al. 2008b, Icarus, 195, 295

Marchis, F., Enriquez, J. E., Emery, J. P., et al. 2012, Icarus, 221, 1130

Marciniak, A., Bartczak, P., Santana-Ros, T., et al. 2012, A\&A, 545, A131

Marciniak, A., Bartczak, P., Müller, T., et al. 2018, A\&A, 610, A7

Margot, J.-L., Pravec, P., Taylor, P., Carry, B., \& Jacobson, S. 2015, Asteroid Systems: Binaries, Triples, and Pairs, eds. P. Michel, F. DeMeo, \& W. F. Bottke (Tucson, AZ: University of Arizona Press), 355

Marsset, M., Carry, B., Yang, B., et al. 2016, IAU Circ., 9282

Marsset, M., Carry, B., Dumas, C., et al. 2017a, A\&A, 604, A64

Marsset, M., Carry, B., Pajuelo, M., et al. 2017b, The Messenger, 169, 29

Masiero, J. R., Mainzer, A. K., Grav, T., et al. 2011, ApJ, 741, 68

Masiero, J. R., Mainzer, A. K., Grav, T., et al. 2012, ApJ, 759, L8

Matter, A., Delbo, M., Ligori, S., Crouzet, N., \& Tanga, P. 2011, Icarus, 215, 47

Merline, W. J., Weidenschilling, S. J., Durda, D. D., et al. 2002, Asteroids III (Tucson, AZ: University of Arizona Press), 289

Morrison, D., \& Zellner, B. 2007, TRIAD Radiometric Diameters and Albedos, NASA Planetary Data System, EAR-A-COMPIL-5-TRIADRAD-V1.0

Mugnier, L. M., Fusco, T., \& Conan, J.-M. 2004, J. Opt. Soc. Am. A, 21, 1841

Nugent, C. R., Mainzer, A., Masiero, J., et al. 2015, ApJ, 814, 117

Pajuelo, M., Carry, B., Vachier, F., et al. 2018, Icarus, 309, 134

Pitjeva, E. V. 2013, Solar Syst. Res., 47, 386

Polishook, D., Brosch, N., Prialnik, D., \& Kaspi, S. 2009, Meteorit. Planet. Sci., 44, 1955

Rayner, J. T., Toomey, D. W., Onaka, P. M., et al. 2003, PASP, 115, 362

Rivkin, A. S. 2012, Icarus, 221, 744

Rivkin, A. S., Thomas, C. A., Howell, E. S., \& Emery, J. P. 2015, AJ, 150, 198 
Rousset, G., Lacombe, F., Puget, P., et al. 2003, SPIE, 4839, 140

Ryan, E. L., \& Woodward, C. E. 2010, AJ, 140, 933

Ryan, E. L., Mizuno, D. R., Shenoy, S. S., et al. 2015, A\&A, 578, A42

Scaltriti, F., \& Zappala, V. 1977, A\&A, 56, 7

Scott, E. R. D. 2007, Ann. Rev. Earth Planet. Sci., 35, 577

Scott, E. R. D., Krot, A. N., \& Sanders, I. S. 2018, ApJ, 854, 164

Sierks, H., Lamy, P., Barbieri, C., et al. 2011, Science, 334, 487

Siltala, L., \& Granvik, M. 2017, Icarus, 297, 149

Somenzi, L., Fienga, A., Laskar, J., \& Kuchynka, P. 2010, Planet. Space Sci., 58, 858

Takir, D., \& Emery, J. P. 2012, Icarus, 219, 641

Takir, D., Emery, J. P., McSween, H. Y., et al. 2013, Meteorit. Planet. Sci., 48 1618

Taylor, M. B. 2005, in Astronomical Data Analysis Software and Systems XIV, eds. P. Shopbell, M. Britton, \& R. Ebert, ASP Conf. Ser., 347, 29

Tedesco, E. F., Egan, M. P., \& Price, S. D. 2004a, MSX Infrared Minor Planet Survey, NASA Planetary Data System, MSX-A-SPIRIT3-5-SBN0003MIMPS-V1.0

Tedesco, E. F., Noah, P. V., Noah, M. C., \& Price, S. D. 2004b, IRAS Minor Planet Survey, NASA Planetary Data System, IRAS-A-FPA-3-RDR-IMPSV6.0

Thalmann, C., Schmid, H. M., Boccaletti, A., et al. 2008, Ground-based and Airborne Instrumentation for Astronomy II, Proc. SPIE, 7014, 70143F

Usui, F., Kuroda, D., Müller, T. G., et al. 2011, PASJ, 63, 1117

Vachier, F., Berthier, J., \& Marchis, F. 2012, A\&A, 543, A68

van Dam, M. A., Le Mignant, D., \& Macintosh, B. 2004, Appl. Opt., 43, 5458

Vernazza, P., Marsset, M., Beck, P., et al. 2016, AJ, 152, 54

Vernazza, P., Broz, M., Drouard, A., et al. 2018, A\&A, 618, A154

Viateau, B. 2000, A\&A, 354, 725

Viikinkoski, M., Kaasalainen, M., \& Durech, J. 2015, A\&A, 576, A8

Viikinkoski, M., Hanuš, J., Kaasalainen, M., Marchis, F., \& Durech, J. 2017, A\&A, 607, A117

Viikinkoski, M., Vernazza, P., Hanus, J., et al. 2018, A\&A 619, L3

Vilas, F. 1994, Icarus, 111, 456

Vilas, F., \& Sykes, M. V. 1996, Icarus, 124, 483

Vilas, F., Larson, S. M., Hatch, E. C., \& Jarvis, K. S. 1993, Icarus, 105, 67

Viswanathan, V., Fienga, A., Gastineau, M., \& Laskar, J. 2017, Notes Scientifiques et Techniques de l'Institut de mécanique céleste, 108, 39

Weidenschilling, S. J., Chapman, C. R., Davis, D. R., et al. 1987, Icarus, 70, 191

Weidenschilling, S. J., Paolicchi, P., \& Zappala, V. 1989, Asteroids II (Tucson, AZ: University of Arizona Press), 643

Weidenschilling, S. J., Chapman, C. R., Davis, D. R., Greenberg, R., \& Levy, D. H. 1990, Icarus, 86, 402

Witasse, O., Lebreton, J.-P., Bird, M. K., et al. 2006, J. Geophys. Res. Planets, 111,7

Yang, B., Wahhaj, Z., Beauvalet, L., et al. 2016, ApJ, 820, L35

Zielenbach, W. 2011, AJ, 142, 120

Zolensky, M. E., Bourcier, W. L., \& Gooding, J. L. 1989, Icarus, 78, 411

Zolensky, M. E., Mittlefehldt, D. W., Lipschutz, M. E., et al. 1997, Geochim. Cosmochim. Acta, 61, 5099

1 Université Côte d'Azur, Observatoire de la Côte d'Azur, CNRS, Laboratoire Lagrange, France

e-mail: benoit. carry@oca.eu
2 IMCCE, Observatoire de Paris, PSL Research University, CNRS, Sorbonne Universités, UPMC Univ Paris 06, Paris, France

3 Astrophysics Research Centre, Queen's University Belfast, BT7 1NN, UK

4 Aix-Marseille Université, CNRS, LAM, Laboratoire d'Astrophysique de Marseille, Marseille, France

5 School of Physical Sciences, The Open University, MK7 6AA, UK

6 Southwest Research Institute, Boulder, CO 80302, USA

7 Université Côte d'Azur, Observatoire de la Côte d'Azur, CNRS, Laboratoire GéoAzur, France

${ }^{8}$ Large Binocular Telescope Observatory, University of Arizona, Tucson, AZ 85721, USA

9 Faculty of Physics, Astronomical Observatory Institute, Adam Mickiewicz University, ul. Słoneczna 36, 60-286 Poznań, Poland

10 Oukaimeden Observatory, High Energy Physics and Astrophysics Laboratory, Cadi Ayyad University, Marrakech, Morocco

11 Institute of Physics, University of Szczecin, Wielkopolska 15, 70-453 Szczecin, Poland

12 Department of Mathematics, Tampere University of Technology, PO Box 553, 33101 Tampere, Finland

13 Max Planck Institute for Astronomy, Königstuhl 17, 69117 Heidelberg, Germany

14 Astronomical Institute, Faculty of Mathematics and Physics, Charles University, V Holešovičkách 2, 18000 Prague, Czech Republic

15 Thirty-Meter-Telescope, 100 West Walnut St, Suite 300, Pasadena, CA 91124, USA

16 Leidos, Starfire Optical Range, AFRL, Kirtland AFB, NM 87117, USA

17 Binary Astronomy, Aurora, CO 80012, USA

18 CdR \& CdL Group: Lightcurves of Minor Planets and Variable Stars, Observatoire de Genève, 1290 Sauverny, Switzerland

19 Jet Propulsion Laboratory, California Institute of Technology, 4800 Oak Grove Drive, Pasadena, CA 91109, USA

20 European Space Agency, ESTEC - Scientific Support Office, Keplerlaan 1, 2200 AG Noordwijk, The Netherlands

21 Observatoire du Bois de Bardon, 16110 Taponnat, France

22 Space sciences, Technologies and Astrophysics Research (STAR) Institute, Université de Liège, Allée du 6 Août 17, 4000 Liège, Belgium

23 SETI Institute, Carl Sagan Center, 189 Bernado Avenue, Mountain View CA 94043, USA

24 Sección Física, Departamento de Ciencias, Pontificia Universidad Católica del Perú, Apartado 1761, Lima, Peru

25 Center for Solar System Studies, 446 Sycamore Ave., Eaton, CO 80615, USA

26 European Southern Observatory (ESO), Alonso de Cordova 3107, 1900 Casilla Vitacura, Santiago, Chile

27 Núcleo de Astronomía, Facultad de Ingeniería y Ciencias, Universidad Diego Portales, Av. Ejercito 441, Santiago, Chile

28 Escuela de Ingeniería Industrial, Facultad de Ingeniería y Ciencias, Universidad Diego Portales, Av. Ejercito 441, Santiago, Chile 
B. Carry et al.: Homogeneous internal structure of CM-like asteroid (41) Daphne

\section{Appendix A: Compilation of diameter and mass estimates}

Table A.1. The diameter estimates $(\mathcal{D})$ of (41) Daphne collected in the literature.

\begin{tabular}{rcrll}
\hline \hline$\#$ & $\begin{array}{c}\mathcal{D} \\
(\mathrm{km})\end{array}$ & $\begin{array}{c}\delta \mathcal{D} \\
(\mathrm{km})\end{array}$ & Method & \multicolumn{1}{c}{ Reference } \\
\hline 1 & 203.00 & 60.90 & STM & Morrison \& Zellner (2007) \\
2 & 174.00 & 35.10 & STM & Tedesco et al. (2004b) \\
3 & 172.43 & 12.24 & STM & Ryan \& Woodward (2010) \\
4 & 207.87 & 31.56 & NEATM & Ryan \& Woodward (2010) \\
5 & 187.00 & 60.00 & LCOCC & Durech et al. (2011) \\
6 & 201.50 & 22.50 & TPM & Matter et al. (2011) \\
7 & 185.50 & 10.50 & TPM & Matter et al. (2011) \\
8 & 179.61 & 7.74 & STM & Usui et al. (2011) \\
9 & 205.50 & 5.64 & NEATM & Masiero et al. (2012) \\
10 & 186.00 & 81.00 & LCIMG & Hanuš et al. (2013b) \\
11 & 198.74 & 185.13 & NEATM & Nugent et al. (2015) \\
12 & 188.00 & 15.00 & ADAM & Hanuš et al. (2017b) \\
13 & 187.00 & 21.50 & ADAM & This work \\
\hline
\end{tabular}

Notes. For each, the $3 \sigma$ uncertainty, method, and bibliographic reference are reported. The methods are ADAM: Multidata 3D Modeling, LCIMG: 3D Model scaled with Imaging, LCOCC: 3D Model scaled with Occultation, NEATM: Near-Earth Asteroid Thermal Model, STM: Standard Thermal Model, TPM: Thermophysical Model.
Table A.2. The mass estimates $(\mathcal{M})$ of (41) Daphne collected in the literature.

\begin{tabular}{rcll}
\hline \hline$\#$ & $\begin{array}{c}\text { Mass }(\mathcal{M}) \\
\left(\times 10^{18} \mathrm{~kg}\right)\end{array}$ & Method & \multicolumn{1}{c}{ Reference } \\
\hline 1 & $10.50 \pm 2.99$ & EPHEM & Fienga et al. (2009) \\
2 & $7.90 \pm 2.37$ & EPHEM & Folkner et al. (2009) \\
3 & $8.43_{-8.43}^{+10.56}$ & EPHEM & Konopliv et al. (2011) \\
4 & $18.20_{-18.20}^{+21.60}$ & DEFL & Zielenbach (2011) \\
5 & $0.30_{-0.30}^{+17.01}$ & DEFL & Zielenbach (2011) \\
6 & $4.76_{-4.76}^{+16.50}$ & DEFL & Zielenbach (2011) \\
7 & $12.10_{-12.10}^{+31.50}$ & DEFL & Zielenbach (2011) \\
8 & $10.20 \pm 3.57$ & EPHEM & Fienga et al. (2011) \\
9 & $7.79 \pm 5.40$ & EPHEM & Kuchynka \& Folkner (2013) \\
10 & $8.29 \pm 2.62$ & EPHEM & Pitjeva (2013) \\
11 & $7.13 \pm 2.01$ & EPHEM & Fienga et al. (2014) \\
12 & $9.35 \pm 4.17$ & DEFL & Goffin (2014) \\
13 & $9.78_{-9.78}^{+13.77}$ & DEFL & Kochetova \& Chernetenko (2014) \\
14 & $4.44 \pm 2.52$ & EPHEM & Fienga (2018, priv. comm.) \\
15 & $6.10 \pm 0.89$ & BGENO & This work \\
\hline
\end{tabular}

Notes. For each, the $3 \sigma$ uncertainty, method, and bibliographic reference are reported. The methods are BGENO: Binary: Genoid, DEFL: Deflection, EPHEM: Ephemeris. 


\section{Appendix B: Shape modeling: data and model predictions}

Table B.1. Date, telescope, camera, number of epochs $\left(\mathcal{N}_{S}\right)$, heliocentric distance $(\Delta)$, range to observer $(r)$, phase angle $(\alpha)$, angular diameter $(\Theta)$, and program ID and Principal Investigator (PI) for each night of AO imaging observations.

\begin{tabular}{|c|c|c|c|c|c|c|c|c|c|c|}
\hline & Date & Telescope & Instrument & $\mathcal{N}_{S}$ & $\begin{array}{c}\Delta \\
(\mathrm{au}) \\
\end{array}$ & $\begin{array}{c}r \\
(\mathrm{au}) \\
\end{array}$ & $\begin{array}{c}\alpha \\
\left(^{\circ}\right) \\
\end{array}$ & $\begin{array}{c}\Theta \\
(\mathrm{mas}) \\
\end{array}$ & Prog. ID & PI \\
\hline 1 & $2002-12-29$ & Keck & NIRC2 & 1 & 2.65 & 1.91 & 16.4 & 135 & C74N2 & J.-L. Margot \\
\hline 2 & $2003-05-06$ & Keck & NIRC2 & 1 & 2.28 & 2.06 & 26.2 & 125 & N17N2 & W. J. Merline \\
\hline 3 & $2008-01-21$ & Keck & NIRC2 & 7 & 2.19 & 1.78 & 26.3 & 144 & DDT & A. Conrad \\
\hline 4 & $2008-03-28$ & Keck & NIRC2 & 10 & 2.06 & 1.09 & 9.1 & 235 & DDT & A. Conrad \\
\hline 5 & 2008-04-23 & Keck & NIRC2 & 5 & 2.03 & 1.06 & 9.9 & 243 & DDT & A. Conrad \\
\hline 6 & $2008-05-10$ & VLT & NACO & 4 & 2.02 & 1.12 & 17.5 & 229 & 281.C-5011 & C. Dumas \\
\hline 7 & 2008-05-14 & VLT & NACO & 2 & 2.02 & 1.14 & 19.1 & 225 & 281.C-5011 & C. Dumas \\
\hline 8 & $2008-05-27$ & VLT & NACO & 2 & 2.01 & 1.23 & 23.6 & 209 & 281.C-5011 & C. Dumas \\
\hline 9 & 2010-11-30 & Keck & NIRC2 & 1 & 3.50 & 2.60 & 7.6 & 99 & U028N2 & F. Marchis \\
\hline 10 & $2017-05-05$ & VLT & SPHERE/IFS & 1 & 2.09 & 1.44 & 25.6 & 179 & 099.D-0098 & J.-L. Beuzit \\
\hline 11 & $2017-05-20$ & VLT & SPHERE/ZIMPOL & 1 & 2.06 & 1.56 & 28.3 & 164 & 199.C-0074 & P. Vernazza \\
\hline 12 & 2018-08-05 & VLT & SPHERE/ZIMPOL & 3 & 2.80 & 1.90 & 11.7 & 136 & 199.C-0074 & P. Vernazza \\
\hline
\end{tabular}

Table B.2. Date, number of positive and negative chords $\left(\#_{\mathrm{p}}\right.$ and $\left.\#_{\mathrm{n}}\right)$, and average uncertainty in seconds $\left(\sigma_{\mathrm{s}}\right)$ and kilometers $\left(\sigma_{\mathrm{km}}\right)$ for each stellar occultation.

\begin{tabular}{lccrccr}
\hline \hline Date & $\begin{array}{c}\mathrm{UT} \\
(\mathrm{h})\end{array}$ & $\#_{\mathrm{p}}$ & $\#_{\mathrm{n}}$ & $\begin{array}{c}\sigma_{\mathrm{s}} \\
(\mathrm{s})\end{array}$ & \multicolumn{1}{c}{$\begin{array}{c}\sigma_{\mathrm{km}} \\
(\mathrm{km})\end{array}$} \\
\hline 1 & $1999-07-02$ & $20: 27$ & 21 & 3 & 1.21 & 46.150 \\
2 & $2008-04-01$ & $18: 12$ & 2 & 1 & 0.50 & 4.814 \\
3 & $2012-01-09$ & $10: 39$ & 1 & 3 & 1.00 & 3.224 \\
4 & $2012-02-23$ & $20: 45$ & 3 & 1 & 0.10 & 1.492 \\
5 & $2012-03-02$ & $18: 49$ & 1 & 4 & 2.00 & 3.411 \\
6 & $2013-03-30$ & $18: 29$ & 2 & 0 & 0.01 & 0.340 \\
7 & $2013-09-05$ & $22: 26$ & 4 & 2 & 0.44 & 7.655 \\
8 & $2013-11-29$ & $19: 16$ & 2 & 0 & 0.26 & 16.458 \\
9 & $2016-01-17$ & $22: 42$ & 19 & 0 & 0.41 & 1104.226 \\
\hline
\end{tabular}


B. Carry et al.: Homogeneous internal structure of CM-like asteroid (41) Daphne

Table B.3. Date, duration $\left(\mathcal{L}\right.$, in hours), number of points $\left(\mathcal{N}_{\mathrm{p}}\right)$, phase angle $(\alpha)$, filter, residual (against the shape model), and observers, for each lightcurve.

\begin{tabular}{|c|c|c|c|c|c|c|c|}
\hline & Date & $\underset{(\mathrm{h})}{\mathcal{L}}$ & $\mathcal{N}_{\mathrm{p}}$ & $\begin{array}{c}\alpha \\
\left(^{\circ}\right)\end{array}$ & Filter & $\begin{array}{l}\mathrm{rms} \\
(\mathrm{mag})\end{array}$ & Observers \\
\hline 1 & $1976-04-26$ & 6.2 & 188 & 18.4 & $\mathrm{~V}$ & 0.024 & Scaltriti \& Zappala (1977) \\
\hline 2 & 1976-05-02 & 6.1 & 163 & 16.8 & V & 0.028 & Scaltriti \& Zappala (1977) \\
\hline 3 & $1976-05-31$ & 3.8 & 130 & 13.8 & $\mathrm{~V}$ & 0.029 & Scaltriti \& Zappala (1977) \\
\hline 4 & 1981-06-16 & 3.7 & 14 & 21.1 & $\mathrm{~V}$ & 0.018 & Weidenschilling et al. (1987) \\
\hline 5 & 1981-06-17 & 1.6 & 7 & 20.9 & $\mathrm{~V}$ & 0.018 & Weidenschilling et al. (1987) \\
\hline 6 & $1981-07-25$ & 4.2 & 34 & 9.8 & $\mathrm{~V}$ & 0.029 & Barucci (1983) \\
\hline 7 & $1981-07-23$ & 4.3 & 58 & 9.8 & $\mathrm{~V}$ & 0.031 & Barucci (1983) \\
\hline 8 & $1981-08-03$ & 4.8 & 78 & 7.4 & $\mathrm{~V}$ & 0.022 & Barucci (1983) \\
\hline 9 & 1981-08-04 & 6.0 & 64 & 7.4 & $\mathrm{~V}$ & 0.033 & Barucci (1983) \\
\hline 10 & 1981-08-06 & 5.6 & 23 & 7.2 & $\mathrm{~V}$ & 0.030 & Weidenschilling et al. (1987) \\
\hline 11 & $1981-11-05$ & 3.6 & 20 & 20.6 & $\mathrm{~V}$ & 0.050 & Weidenschilling et al. (1987) \\
\hline 12 & 1981-12-01 & 1.3 & 7 & 18.8 & $\mathrm{~V}$ & 0.054 & Weidenschilling et al. (1987) \\
\hline 13 & 1981-12-02 & 5.9 & 12 & 18.7 & $\mathrm{~V}$ & 0.028 & Weidenschilling et al. (1987) \\
\hline 14 & 1982-09-29 & 4.8 & 20 & 7.5 & $\mathrm{~V}$ & 0.017 & Weidenschilling et al. (1987) \\
\hline 15 & $1982-10-26$ & 4.8 & 35 & 3.7 & $\mathrm{~V}$ & 0.033 & Barucci (1983) \\
\hline 16 & 1983-10-11 & 1.0 & 5 & 16.7 & $\mathrm{~V}$ & 0.035 & Weidenschilling et al. (1987) \\
\hline 17 & $1983-10-15$ & 1.8 & 7 & 16.4 & V & 0.017 & Weidenschilling et al. (1987) \\
\hline 18 & 1983-11-12 & 4.1 & 24 & 12.2 & $\mathrm{~V}$ & 0.015 & Weidenschilling et al. (1987) \\
\hline 19 & $1983-11-14$ & 5.8 & 14 & 11.8 & $\mathrm{~V}$ & 0.024 & Weidenschilling et al. (1987) \\
\hline 20 & $1983-12-28$ & 6.0 & 83 & 8.1 & $\mathrm{~V}$ & 0.068 & Barucci (1983) \\
\hline 21 & 1983-12-29 & 0.8 & 15 & 8.2 & $\mathrm{~V}$ & 0.032 & Barucci (1983) \\
\hline 22 & $1983-12-30$ & 4.0 & 77 & 8.4 & $\mathrm{~V}$ & 0.047 & Barucci (1983) \\
\hline 23 & 1984-02-21 & 4.8 & 34 & 18.1 & $\mathrm{~V}$ & 0.028 & Weidenschilling et al. (1987) \\
\hline 24 & 1985-01-18 & 2.2 & 21 & 25.8 & $\mathrm{~V}$ & 0.020 & Weidenschilling et al. (1987) \\
\hline 25 & 1985-01-19 & 3.9 & 21 & 25.7 & $\mathrm{~V}$ & 0.023 & Weidenschilling et al. (1987) \\
\hline 26 & 1985-04-11 & 5.1 & 13 & 5.8 & $\mathrm{~V}$ & 0.014 & Weidenschilling et al. (1987) \\
\hline 27 & $1987-10-17$ & 5.8 & 25 & 8.7 & $\mathrm{~V}$ & 0.019 & Weidenschilling et al. (1987) \\
\hline 28 & $1988-12-20$ & 5.9 & 37 & 11.5 & $\mathrm{~V}$ & 0.042 & Weidenschilling et al. (1987) \\
\hline 29 & $1988-12-21$ & 5.8 & 15 & 11.2 & $\mathrm{~V}$ & 0.035 & Weidenschilling et al. (1987) \\
\hline 30 & $2001-11-21$ & 7.2 & 103 & 5.4 & $\mathrm{~V}$ & 0.023 & L. Bernasconi \\
\hline 31 & $2001-11-22$ & 8.4 & 107 & 5.4 & $\mathrm{~V}$ & 0.023 & L. Bernasconi \\
\hline 32 & $2001-11-23$ & 7.0 & 102 & 5.5 & $\mathrm{~V}$ & 0.027 & L. Bernasconi \\
\hline 33 & $2001-11-24$ & 8.8 & 87 & 5.5 & $\mathrm{~V}$ & 0.022 & L. Bernasconi \\
\hline 34 & $2008-06-25$ & 2.3 & 49 & 12.9 & Clear & 0.018 & SuperWASP - J. Grice \\
\hline 35 & $2009-10-16$ & 3.0 & 45 & 4.3 & Clear & 0.021 & SuperWASP - J. Grice \\
\hline 36 & $2009-09-25$ & 2.7 & 117 & 8.2 & $\mathrm{~V}$ & 0.019 & ASTEP \\
\hline 37 & $2009-10-16$ & 2.8 & 34 & 11.2 & Clear & 0.015 & SuperWASP - J. Grice \\
\hline 38 & 2017-01-08 & 2.3 & 31 & 22.0 & $\mathrm{R}$ & 0.024 & Vachier, Klotz, Teng, Peyrot, Thierry, Berthier \\
\hline 39 & 2018-07-09 & 10.6 & 651 & 19.1 & $\mathrm{R}$ & 0.023 & E. Jehin \\
\hline 40 & 2018-07-10 & 6.0 & 507 & 18.9 & $\mathrm{R}$ & 0.024 & E. Jehin \\
\hline 41 & 2018-08-03 & 3.6 & 200 & 12.4 & $\mathrm{R}$ & 0.023 & S. Fauvaud \\
\hline 42 & 2018-08-04 & 6.2 & 200 & 12.1 & $\mathrm{R}$ & 0.020 & S. Fauvaud \\
\hline 43 & 2018-08-05 & 2.1 & 200 & 11.7 & $\mathrm{R}$ & 0.019 & S. Fauvaud \\
\hline 44 & 2018-08-05 & 1.9 & 137 & 11.4 & $\mathrm{R}$ & 0.013 & S. Fauvaud \\
\hline 45 & 2018-08-06 & 1.8 & 136 & 11.1 & $\mathrm{R}$ & 0.012 & S. Fauvaud \\
\hline 46 & 2018-08-11 & 1.6 & 139 & 9.3 & $\mathrm{R}$ & 0.009 & S. Fauvaud \\
\hline 47 & 2018-08-14 & 1.8 & 139 & 8.2 & $\mathrm{R}$ & 0.008 & S. Fauvaud \\
\hline 48 & $2018-08-15$ & 1.4 & 105 & 7.8 & $\mathrm{R}$ & 0.007 & S. Fauvaud \\
\hline 49 & 2018-11-19 & 3.1 & 52 & 18.6 & $\mathrm{R}$ & 0.016 & Vachier, Klotz, Teng, Peyrot, Thierry, Berthier \\
\hline
\end{tabular}




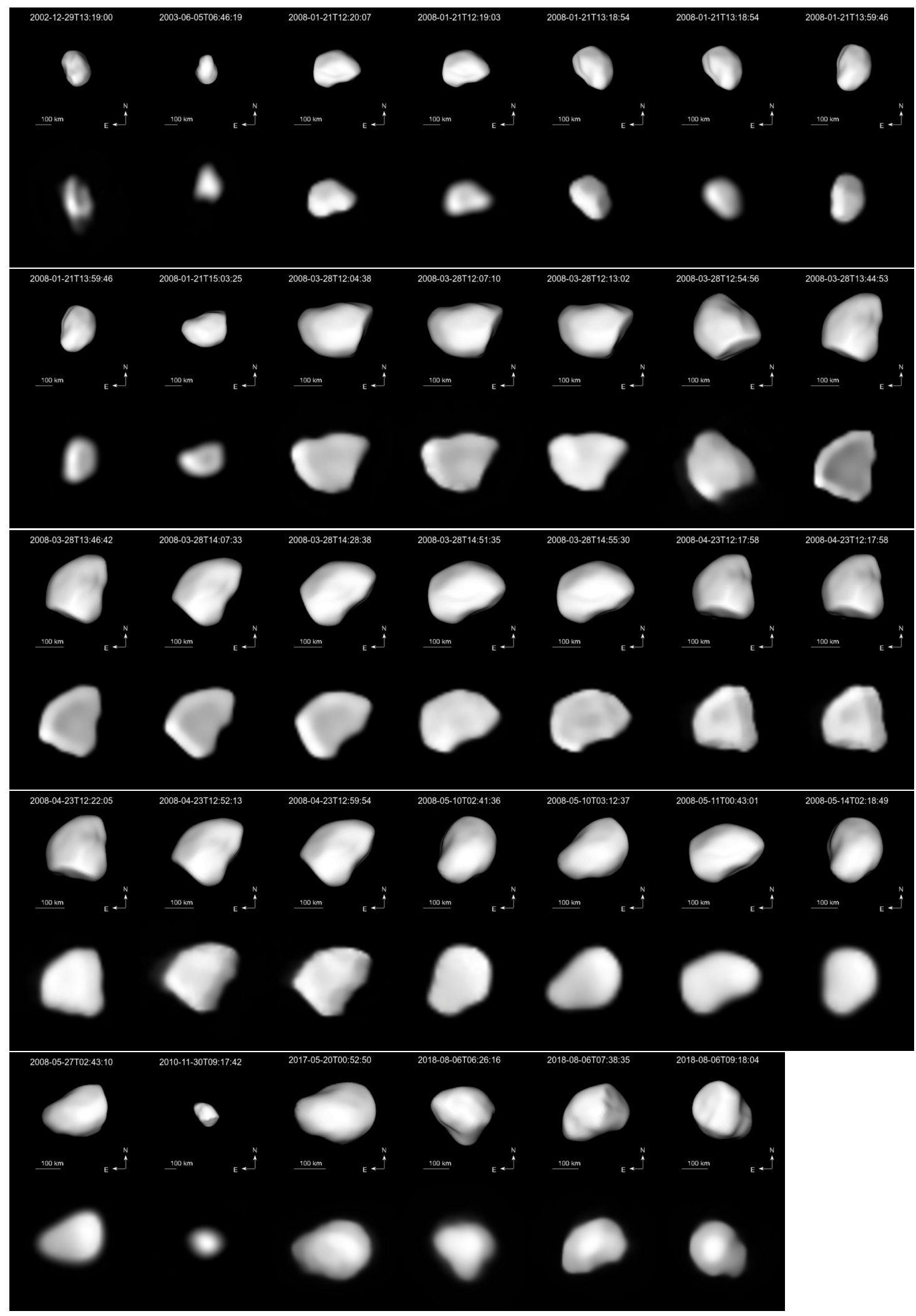

Fig. B.1. Comparison of the shape model (top image of each row), oriented and projected on the plane of the sky at the epoch of each disk-resolved observation (bottom image of each row). The brighter ring present in some images (e.g., second row, last column) is an artifact from deconvolution. 


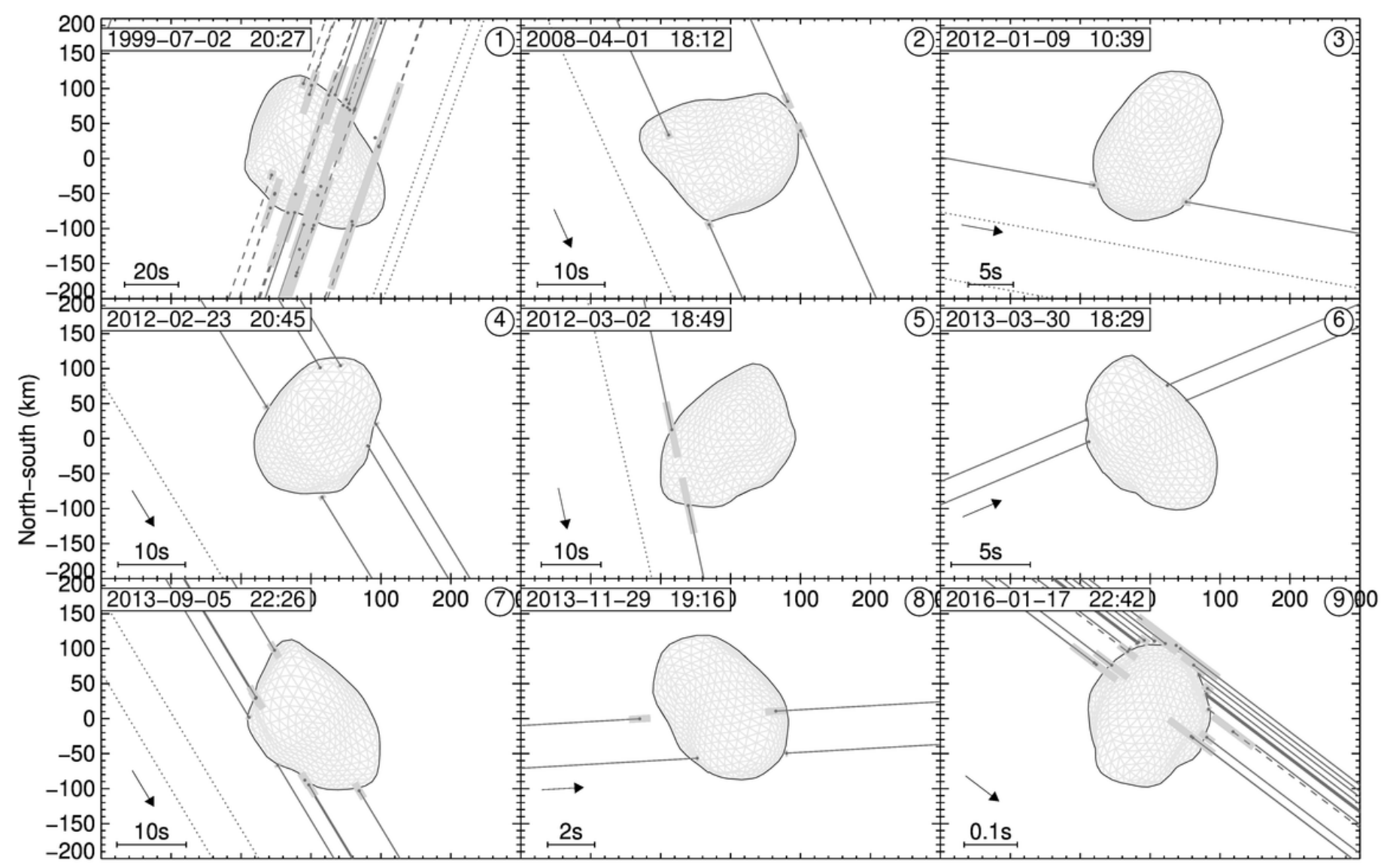

East-West $(\mathrm{km})$

Fig. B.2. The eight stellar occultations by Daphne, compared with the shape model (profile in black and facets in light gray) projected on the plane of the sky at the time of the occultation. Negative chords are represented by dotted lines, visual timings by dashed lines, and electronic timings by solid lines. Disappearance and reappearances timings are marked by filled circles, and their uncertainty by gray rectangles. 


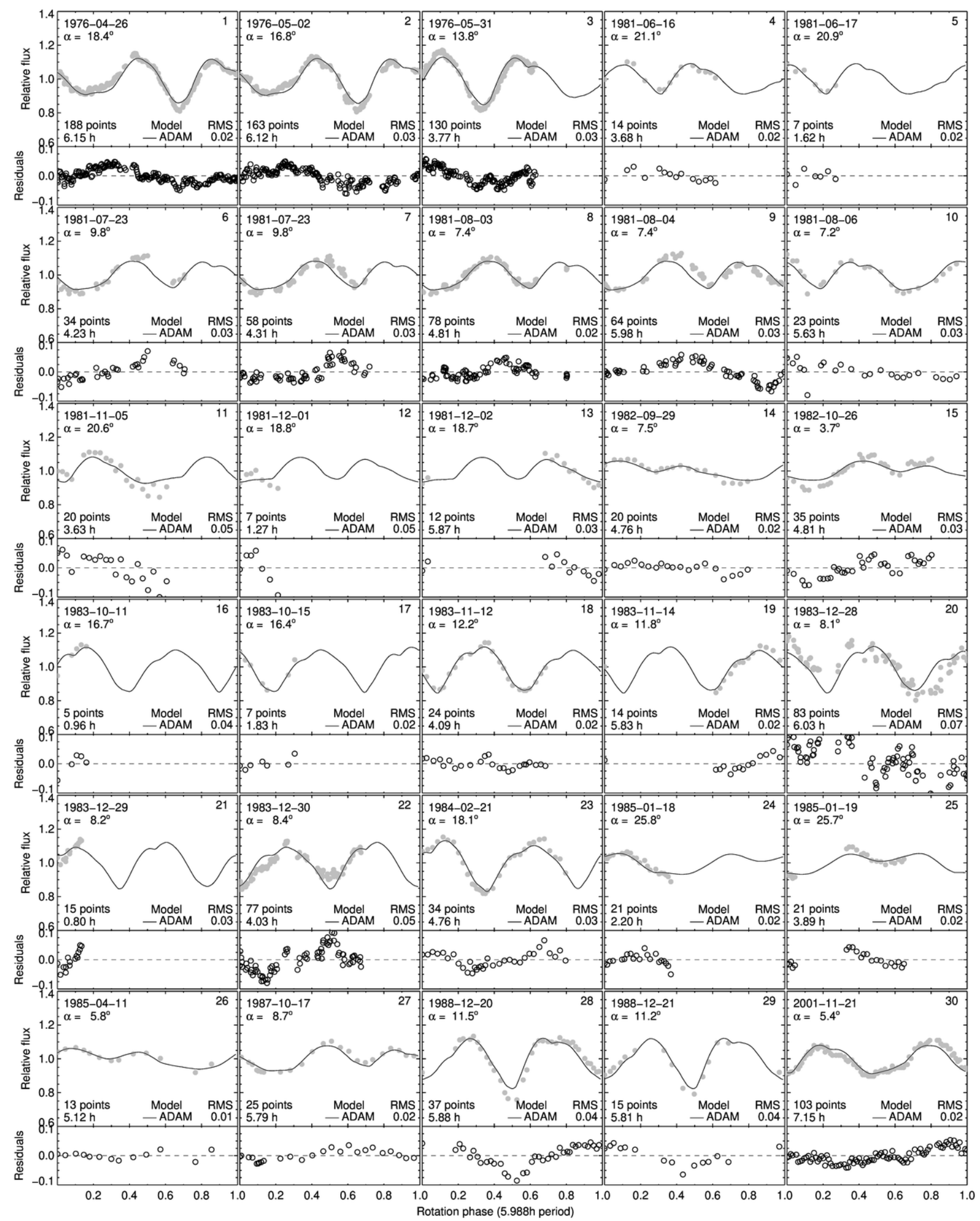

Fig. B.3. The optical lightcurves of Daphne (grey spheres), compared with the synthetic lightcurves generated with the shape model (black lines). On each panel, the observing date, number of points, duration of the lightcurve (in hours), and rms residuals between the observations and the synthetic lightcurves are displayed. Measurement uncertainties are seldom provided by the observers but can be estimated from the spread of measurements. 
B. Carry et al.: Homogeneous internal structure of CM-like asteroid (41) Daphne

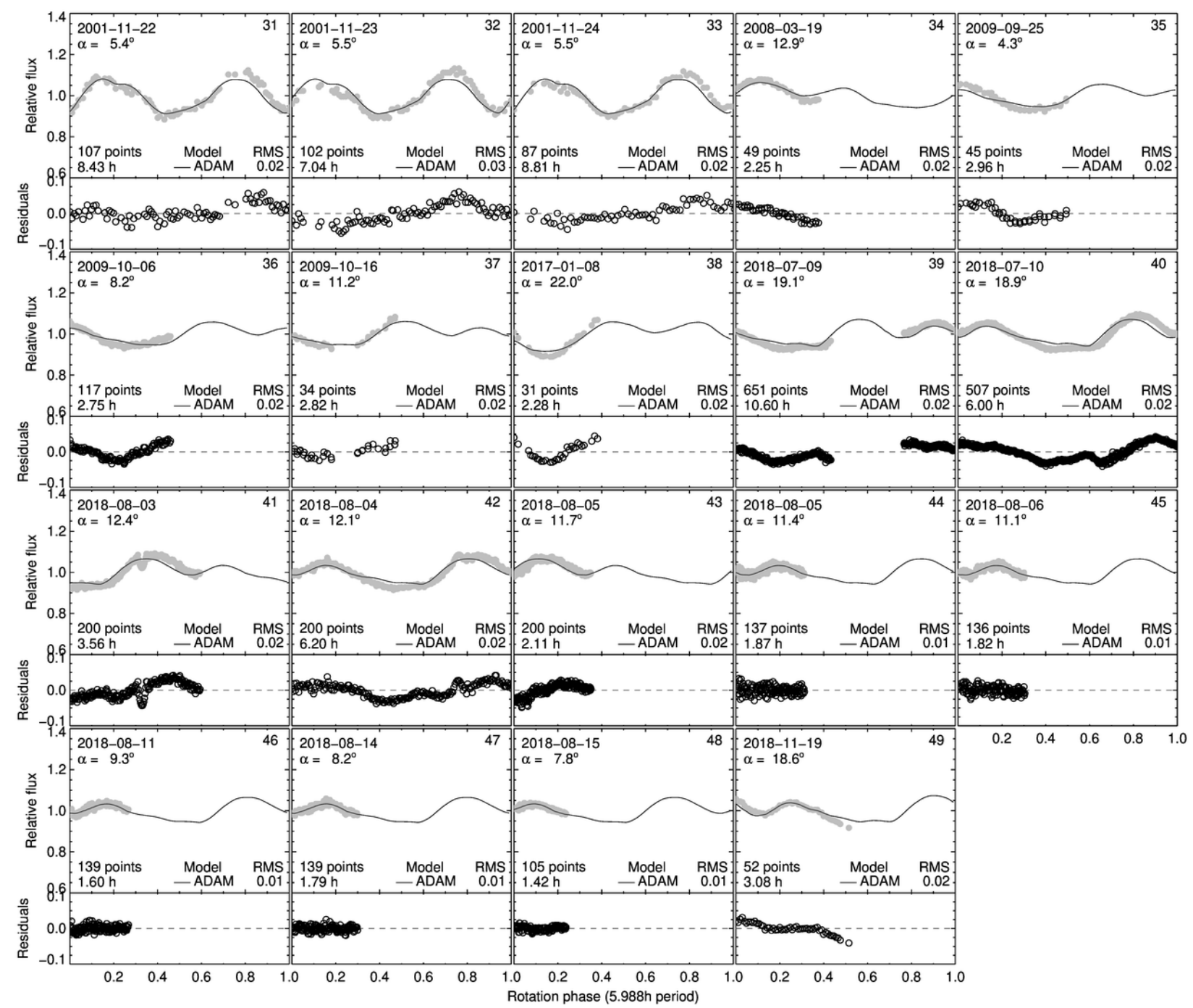

Fig. B.3. continued. 


\section{Appendix C: Magnitude and position of the satellite}

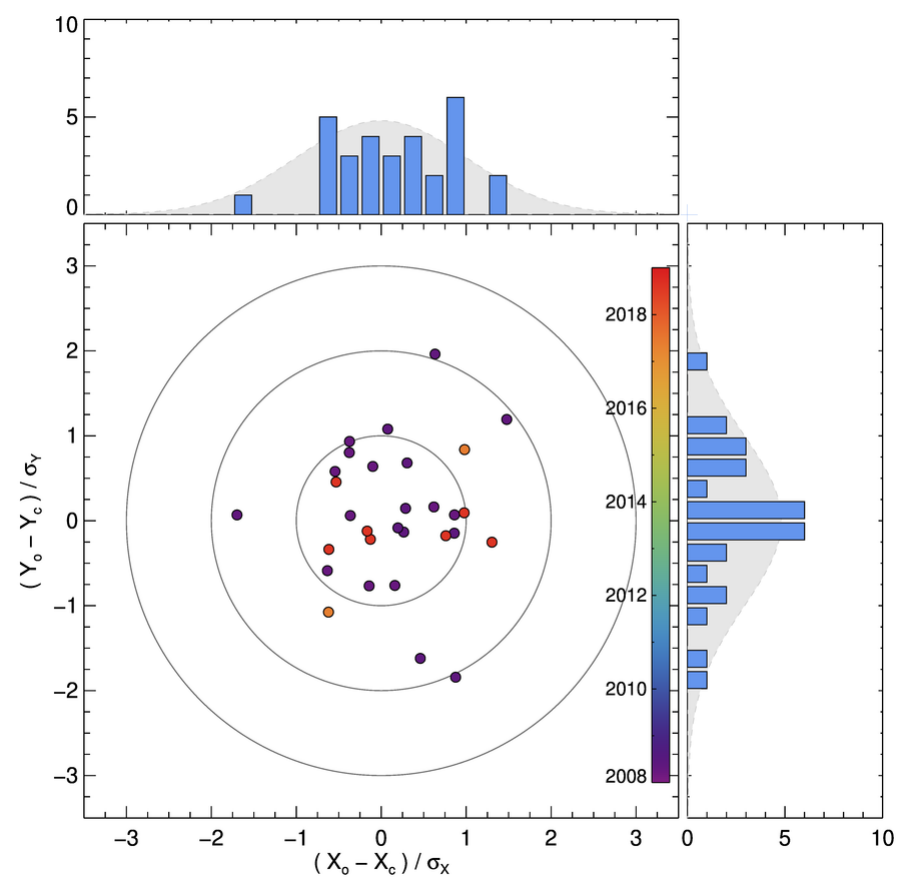

Fig. C.1. Distribution of residuals for the satellite between the observed (index o) and predicted (index c) positions, normalized by the uncertainty on the measured positions $(\sigma)$, and color-coded by observing epoch. X stands for right ascension and $\mathrm{Y}$ for declination. The three large gray circles represent the 1,2 , and $3 \sigma$ limits (typically 10 mas at $1 \sigma)$. Top panel: histogram of residuals along X. Right panel: residuals along Y. The light gray Gaussian in the background has a standard deviation of one.

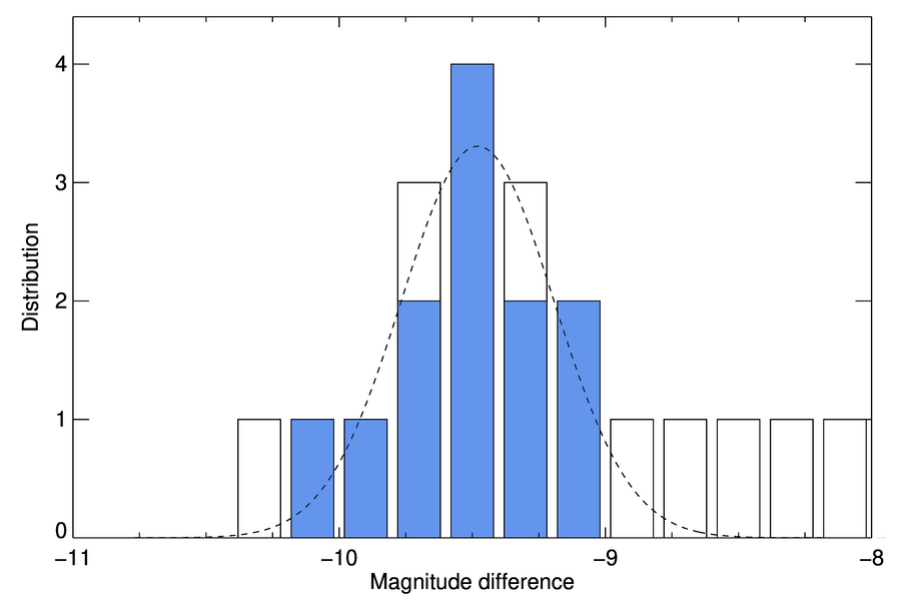

Fig. C.2. Distribution of the magnitude differences between Daphne and its satellite. The open bars represent all measurements, and the blue bars those more precise than 0.75 magnitude. The dashed black line represents the normal distribution fit to our results, with a mean and standard deviation of $9.49 \pm 0.32$. 
Table C.1. Astrometry of the satellite of Daphne.

\begin{tabular}{|c|c|c|c|c|c|c|c|c|c|c|c|}
\hline Date & UTC & Tel. & Cam. & Filter & $\begin{array}{c}X_{\mathrm{o}} \\
\text { (mas) }\end{array}$ & $\begin{array}{c}Y_{\mathrm{o}} \\
\text { (mas) }\end{array}$ & $\begin{array}{c}X_{\mathrm{o}-\mathrm{c}} \\
\text { (mas) }\end{array}$ & $\begin{array}{c}Y_{\mathrm{o}-\mathrm{c}} \\
\text { (mas) }\end{array}$ & $\begin{array}{c}\sigma \\
\text { (mas) }\end{array}$ & $\begin{array}{c}\Delta M \\
(\mathrm{mag})\end{array}$ & $\begin{array}{c}\delta M \\
(\mathrm{mag})\end{array}$ \\
\hline $2008-03-28$ & $12: 05: 30.1$ & Keck & NIRC2 & $\mathrm{J}$ & -554 & 60 & -6 & -5 & 9.94 & -9.59 & 0.85 \\
\hline 2008-03-28 & $12: 07: 59.5$ & Keck & NIRC2 & $\mathbf{J}$ & -549 & 53 & -1 & -7 & 9.94 & -10.06 & 0.64 \\
\hline $2008-03-28$ & $12: 56: 28.9$ & Keck & NIRC2 & $\mathrm{H}$ & -531 & -19 & -5 & 5 & 9.94 & - & - \\
\hline $2008-03-28$ & $13: 45: 16.2$ & Keck & NIRC2 & $\mathrm{H}$ & -476 & -110 & 8 & 0 & 9.94 & - & - \\
\hline $2008-03-28$ & $13: 48: 16.8$ & Keck & NIRC2 & $\mathrm{H}$ & -475 & -115 & 6 & 1 & 9.94 & -9.70 & 0.14 \\
\hline $2008-03-28$ & 14:09:03.8 & Keck & NIRC2 & $\mathrm{H}$ & -457 & -160 & 1 & -7 & 9.94 & -9.10 & 2.19 \\
\hline $2008-03-28$ & 14:30:11.3 & Keck & NIRC2 & $\mathrm{H}$ & -435 & -187 & -3 & 0 & 9.94 & - & - \\
\hline 2008-03-28 & 14:53:08.0 & Keck & NIRC2 & $\mathrm{Kp}$ & -403 & -215 & -3 & 9 & 9.94 & -9.84 & 0.73 \\
\hline $2008-03-28$ & $14: 57: 06.5$ & Keck & NIRC2 & $\mathrm{Kp}$ & -397 & -222 & -3 & 7 & 9.94 & - & - \\
\hline $2008-03-28$ & $15: 00: 34.9$ & Keck & NIRC2 & $\mathrm{H}$ & -389 & -229 & 0 & 6 & 9.94 & - & - \\
\hline $2008-04-23$ & $12: 17: 26.4$ & Keck & NIRC2 & $\mathrm{H}$ & -424 & 376 & -16 & 0 & 9.94 & - & - \\
\hline 2008-04-23 & $12: 53: 37.9$ & Keck & NIRC2 & $\mathrm{H}$ & -453 & 361 & 6 & 19 & 9.94 & - & - \\
\hline 2008-04-23 & $12: 58: 51.4$ & Keck & NIRC2 & $\mathrm{H}$ & -452 & 347 & 14 & 11 & 9.94 & - & - \\
\hline $2008-05-10$ & $02: 43: 04.5$ & VLT & NACO & $\mathrm{H}$ & 550 & -177 & 3 & -1 & 13.24 & -9.56 & 0.78 \\
\hline 2008-05-10 & $02: 47: 42.2$ & VLT & NACO & $\mathrm{H}$ & 552 & -167 & 3 & 1 & 13.24 & -9.53 & 1.49 \\
\hline 2008-05-10 & 03:14:05.9 & VLT & NACO & $\mathrm{H}$ & 559 & -124 & 4 & 9 & 13.24 & -9.52 & 0.84 \\
\hline 2008-05-10 & 03:18:44.1 & VLT & NACO & $\mathrm{H}$ & 556 & -112 & 1 & 14 & 13.24 & -9.29 & 1.13 \\
\hline 2008-05-14 & 02:20:19.1 & VLT & NACO & $\mathrm{H}$ & -516 & 142 & 11 & -24 & 13.24 & -9.28 & 0.37 \\
\hline $2008-05-14$ & $02: 24: 59.4$ & VLT & NACO & $\mathrm{H}$ & -523 & 139 & 6 & -21 & 13.24 & -9.02 & 0.19 \\
\hline $2008-05-27$ & $02: 44: 38.8$ & VLT & NACO & $\mathrm{H}$ & 449 & -256 & 11 & -1 & 13.24 & - & - \\
\hline $2008-05-27$ & $02: 49: 12.7$ & VLT & NACO & $\mathrm{H}$ & 445 & -251 & 2 & -1 & 13.24 & -9.68 & 0.65 \\
\hline 2017-05-05 & $22: 51: 25.1$ & VLT & IFS & YJH & 404 & 158 & -6 & -10 & 10.00 & - & - \\
\hline $2017-05-20$ & 01:01:27.4 & VLT & ZIMPOL & $\mathrm{R}$ & -360 & 35 & 9 & 8 & 10.00 & - & - \\
\hline 2018-08-06 & $06: 28: 30.9$ & VLT & ZIMPOL & $\mathrm{R}$ & -268 & 208 & -5 & 4 & 10.00 & - & - \\
\hline 2018-08-06 & 06:33:09.9 & VLT & ZIMPOL & $\mathrm{R}$ & -266 & 203 & -6 & -3 & 10.00 & - & - \\
\hline 2018-08-06 & 07:40:49.4 & VLT & ZIMPOL & $\mathrm{R}$ & -193 & 244 & 7 & -1 & 10.00 & - & - \\
\hline 2018-08-06 & $07: 45: 25.2$ & VLT & ZIMPOL & $\mathrm{R}$ & -186 & 249 & 9 & 0 & 10.00 & - & - \\
\hline $2018-08-06$ & $07: 59: 15.8$ & VLT & ZIMPOL & $\mathrm{R}$ & -169 & 251 & 13 & -2 & 10.00 & -9.97 & 0.63 \\
\hline 2018-08-06 & 09:20:18.1 & VLT & ZIMPOL & $\mathrm{R}$ & -92 & 271 & -1 & -2 & 10.00 & - & - \\
\hline 2018-08-06 & $09: 24: 57.2$ & VLT & ZIMPOL & $\mathrm{R}$ & -87 & 272 & -1 & -1 & 10.00 & -10.02 & 1.34 \\
\hline & & & & & \multirow{2}{*}{\multicolumn{2}{|c|}{ Average }} & 2 & 0 & 15 & -9.49 & 0.28 \\
\hline & & & & & & & Star & 9 & 2 & 0.32 & 0.18 \\
\hline
\end{tabular}

Notes. Date, mid-observing time (UTC), telescope, camera, filter, astrometry ( $X$ is aligned with right ascension, and $Y$ with declination, and o and $\mathrm{c}$ indices stand for observed and computed positions), and photometry (magnitude difference $\Delta M$ with uncertainty $\delta M$ ). 


\section{Appendix D: Mass and diameter of $\mathrm{Ch}$ and $\mathrm{Cgh}$ asteroids}

Table D.1. Dynamical class (IMB, MMB, and OMB stand for inner, middle, and outer belt respectively), taxonomic class, mass $(\mathcal{M})$, diameter $(\mathcal{D})$, and density $(\rho)$ of $\mathrm{Ch} / \mathrm{Cgh}$ asteroids used in this work, compiled following the recipes described in Carry (2012).

\begin{tabular}{|c|c|c|c|c|c|c|c|c|c|}
\hline$\#$ & Name & Dyn. & Taxo. & $\begin{array}{c}\mathcal{M} \\
(\mathrm{kg})\end{array}$ & $\begin{array}{l}\sigma_{\mathcal{M}} \\
(\mathrm{kg})\end{array}$ & $\begin{array}{c}\mathcal{D} \\
(\mathrm{km})\end{array}$ & $\begin{array}{c}\sigma_{\mathcal{D}} \\
(\mathrm{km})\end{array}$ & $\begin{array}{c}\rho \\
\left(\mathrm{g} \mathrm{cm}^{-3}\right)\end{array}$ & $\begin{array}{c}\sigma_{\rho} \\
\left(\mathrm{g} \mathrm{cm}^{-3}\right)\end{array}$ \\
\hline 13 & Egeria & MMB & $\mathrm{Ch}$ & $8.42 \times 10^{18}$ & $2.28 \times 10^{18}$ & 206.56 & 8.22 & 1.82 & 0.54 \\
\hline 19 & Fortuna & IMB & $\mathrm{Ch}$ & $9.03 \times 10^{18}$ & $7.13 \times 10^{17}$ & 207.54 & 5.30 & 1.93 & 0.21 \\
\hline 34 & Circe & MMB & $\mathrm{Ch}$ & $4.16 \times 10^{18}$ & $6.27 \times 10^{17}$ & 114.34 & 3.23 & 5.31 & 0.92 \\
\hline 38 & Leda & MMB & $\mathrm{Cgh}$ & $3.18 \times 10^{18}$ & $5.97 \times 10^{17}$ & 118.62 & 4.22 & 3.64 & 0.79 \\
\hline 41 & Daphne & MMB & $\mathrm{Ch}$ & $6.10 \times 10^{18}$ & $7.50 \times 10^{17}$ & 187.00 & 7.50 & 1.77 & 0.22 \\
\hline 48 & Doris & OMB & $\mathrm{Ch}$ & $7.71 \times 10^{18}$ & $1.57 \times 10^{18}$ & 208.37 & 12.02 & 1.63 & 0.44 \\
\hline 49 & Pales & OMB & $\mathrm{Ch}$ & $4.10 \times 10^{18}$ & $1.77 \times 10^{18}$ & 149.97 & 3.78 & 2.32 & 1.02 \\
\hline 50 & Virginia & MMB & $\mathrm{Ch}$ & $6.26 \times 10^{17}$ & $2.06 \times 10^{17}$ & 88.51 & 4.65 & 1.72 & 0.63 \\
\hline 51 & Nemausa & IMB & $\mathrm{Cgh}$ & $2.62 \times 10^{18}$ & $8.19 \times 10^{17}$ & 146.80 & 1.56 & 1.58 & 0.50 \\
\hline 54 & Alexandra & MMB & $\mathrm{Cgh}$ & $1.72 \times 10^{18}$ & $5.89 \times 10^{17}$ & 150.12 & 8.14 & 0.97 & 0.37 \\
\hline 58 & Concordia & MMB & $\mathrm{Ch}$ & $1.28 \times 10^{17}$ & $6.41 \times 10^{16}$ & 94.84 & 1.57 & 0.29 & 0.14 \\
\hline 62 & Erato & OMB & $\mathrm{Ch}$ & $1.38 \times 10^{17}$ & $6.91 \times 10^{16}$ & 96.06 & 14.26 & 0.30 & 0.20 \\
\hline 70 & Panopaea & MMB & $\mathrm{Cgh}$ & $3.64 \times 10^{18}$ & $5.94 \times 10^{17}$ & 135.34 & 5.47 & 2.81 & 0.57 \\
\hline 78 & Diana & MMB & $\mathrm{Ch}$ & $8.99 \times 10^{17}$ & $5.31 \times 10^{17}$ & 125.33 & 4.77 & 0.87 & 0.53 \\
\hline 84 & Klio & IMB & $\mathrm{Ch}$ & $6.63 \times 10^{17}$ & $1.51 \times 10^{17}$ & 78.76 & 1.12 & 2.59 & 0.60 \\
\hline 91 & Aegina & MMB & $\mathrm{Ch}$ & $6.07 \times 10^{17}$ & $2.98 \times 10^{17}$ & 103.67 & 4.86 & 1.04 & 0.53 \\
\hline 95 & Arethusa & OMB & $\mathrm{Ch}$ & $4.18 \times 10^{18}$ & $7.95 \times 10^{17}$ & 138.07 & 10.19 & 3.03 & 0.89 \\
\hline 98 & Ianthe & MMB & $\mathrm{Ch}$ & $8.53 \times 10^{17}$ & $1.65 \times 10^{17}$ & 105.48 & 3.29 & 1.39 & 0.30 \\
\hline 104 & Klymene & OMB & $\mathrm{Ch}$ & $1.91 \times 10^{18}$ & $6.59 \times 10^{17}$ & 130.48 & 5.85 & 1.64 & 0.61 \\
\hline 105 & Artemis & IMB & $\mathrm{Ch}$ & $1.43 \times 10^{18}$ & $4.14 \times 10^{17}$ & 122.44 & 2.70 & 1.49 & 0.44 \\
\hline 106 & Dione & OMB & $\mathrm{Cgh}$ & $4.86 \times 10^{18}$ & $2.18 \times 10^{18}$ & 146.60 & 3.41 & 2.95 & 1.34 \\
\hline 109 & Felicitas & MMB & $\mathrm{Ch}$ & $1.69 \times 10^{17}$ & $3.50 \times 10^{16}$ & 82.38 & 5.09 & 0.58 & 0.16 \\
\hline 111 & Ate & MMB & $\mathrm{Ch}$ & $1.87 \times 10^{18}$ & $3.48 \times 10^{17}$ & 134.28 & 2.08 & 1.48 & 0.28 \\
\hline 112 & Iphigenia & IMB & $\mathrm{Ch}$ & $4.52 \times 10^{17}$ & $2.12 \times 10^{17}$ & 71.06 & 0.67 & 2.41 & 1.13 \\
\hline 121 & Hermione & Cybele & $\mathrm{Ch}$ & $4.94 \times 10^{18}$ & $4.21 \times 10^{17}$ & 196.69 & 8.36 & 1.24 & 0.19 \\
\hline 127 & Johanna & MMB & $\mathrm{Ch}$ & $2.39 \times 10^{18}$ & $9.97 \times 10^{17}$ & 120.04 & 0.05 & 2.64 & 1.10 \\
\hline 130 & Elektra & OMB & $\mathrm{Ch}$ & $6.20 \times 10^{18}$ & $4.45 \times 10^{16}$ & 189.56 & 6.24 & 1.74 & 0.17 \\
\hline 134 & Sophrosyne & MMB & $\mathrm{Ch}$ & $9.52 \times 10^{17}$ & $1.24 \times 10^{18}$ & 107.02 & 9.63 & 1.48 & 1.97 \\
\hline 141 & Lumen & MMB & $\mathrm{Ch}$ & $3.27 \times 10^{18}$ & $9.33 \times 10^{17}$ & 132.24 & 1.73 & 2.70 & 0.78 \\
\hline 144 & Vibilia & MMB & $\mathrm{Ch}$ & $3.75 \times 10^{18}$ & $6.95 \times 10^{17}$ & 141.86 & 1.42 & 2.51 & 0.47 \\
\hline 145 & Adeona & MMB & $\mathrm{Ch}$ & $2.32 \times 10^{18}$ & $2.11 \times 10^{17}$ & 149.48 & 5.72 & 1.33 & 0.19 \\
\hline 146 & Lucina & MMB & $\mathrm{Ch}$ & $1.18 \times 10^{17}$ & $5.91 \times 10^{16}$ & 129.10 & 3.94 & 0.10 & 0.05 \\
\hline 156 & Xanthippe & MMB & $\mathrm{Ch}$ & $1.28 \times 10^{18}$ & $2.52 \times 10^{18}$ & 115.93 & 4.26 & 1.57 & 3.10 \\
\hline 159 & Aemilia & OMB & $\mathrm{Ch}$ & $4.18 \times 10^{18}$ & $5.97 \times 10^{17}$ & 133.93 & 7.59 & 3.32 & 0.74 \\
\hline 162 & Laurentia & $\mathrm{OMB}$ & $\mathrm{Ch}$ & $3.36 \times 10^{17}$ & $1.67 \times 10^{17}$ & 98.65 & 1.95 & 0.67 & 0.33 \\
\hline 163 & Erigone & IMB & $\mathrm{Ch}$ & $5.79 \times 10^{17}$ & $2.43 \times 10^{17}$ & 72.29 & 1.22 & 2.93 & 1.24 \\
\hline 168 & Sibylla & Cybele & $\mathrm{Ch}$ & $5.75 \times 10^{18}$ & $1.68 \times 10^{18}$ & 148.97 & 4.51 & 3.32 & 1.01 \\
\hline 176 & Iduna & OMB & $\mathrm{Ch}$ & $3.36 \times 10^{17}$ & $1.68 \times 10^{17}$ & 119.40 & 3.57 & 0.38 & 0.19 \\
\hline 187 & Lamberta & MMB & $\mathrm{Ch}$ & $8.05 \times 10^{17}$ & $7.07 \times 10^{17}$ & 131.18 & 1.21 & 0.68 & 0.60 \\
\hline 195 & Eurykleia & OMB & $\mathrm{Ch}$ & $1.44 \times 10^{17}$ & $7.19 \times 10^{16}$ & 90.22 & 4.21 & 0.37 & 0.19 \\
\hline 200 & Dynamene & MMB & $\mathrm{Ch}$ & $1.29 \times 10^{18}$ & $8.87 \times 10^{17}$ & 131.11 & 2.95 & 1.09 & 0.76 \\
\hline 205 & Martha & MMB & $\mathrm{Ch}$ & $1.19 \times 10^{17}$ & $5.94 \times 10^{16}$ & 69.44 & 7.11 & 0.68 & 0.40 \\
\hline 211 & Isolda & OMB & $\mathrm{Ch}$ & $3.31 \times 10^{18}$ & $1.27 \times 10^{18}$ & 151.33 & 4.77 & 1.83 & 0.72 \\
\hline 238 & Hypatia & $\mathrm{OMB}$ & $\mathrm{Ch}$ & $3.31 \times 10^{18}$ & $9.25 \times 10^{17}$ & 144.90 & 2.21 & 2.08 & 0.59 \\
\hline 266 & Aline & MMB & $\mathrm{Ch}$ & $1.43 \times 10^{18}$ & $6.93 \times 10^{17}$ & 104.96 & 4.95 & 2.36 & 1.19 \\
\hline 303 & Josephina & OMB & $\mathrm{Ch}$ & $1.31 \times 10^{17}$ & $6.57 \times 10^{16}$ & 100.64 & 7.25 & 0.25 & 0.13 \\
\hline 345 & Tercidina & IMB & $\mathrm{Ch}$ & $1.17 \times 10^{18}$ & $5.11 \times 10^{17}$ & 99.09 & 1.40 & 2.30 & 1.01 \\
\hline 350 & Ornamenta & OMB & $\mathrm{Ch}$ & $2.59 \times 10^{17}$ & $1.29 \times 10^{17}$ & 111.97 & 8.43 & 0.35 & 0.19 \\
\hline 356 & Liguria & MMB & $\mathrm{Ch}$ & $2.62 \times 10^{18}$ & $2.16 \times 10^{18}$ & 139.97 & 6.22 & 1.82 & 1.52 \\
\hline 358 & Apollonia & OMB & $\mathrm{Ch}$ & $1.60 \times 10^{17}$ & $7.99 \times 10^{16}$ & 89.11 & 1.78 & 0.43 & 0.22 \\
\hline
\end{tabular}

Notes. Individual diameter and mass estimates are listed in Tables D.2 and D.3. 
Table D.1. continued.

\begin{tabular}{|c|c|c|c|c|c|c|c|c|c|}
\hline \# & Name & Dyn. & Taxo. & $\begin{array}{c}\mathcal{M} \\
(\mathrm{kg})\end{array}$ & $\begin{array}{l}\sigma_{\mathcal{M}} \\
(\mathrm{kg})\end{array}$ & $\begin{array}{c}\mathcal{D} \\
(\mathrm{km})\end{array}$ & $\begin{array}{c}\sigma_{\mathcal{D}} \\
(\mathrm{km})\end{array}$ & $\begin{array}{c}\rho \\
\left(\mathrm{g} \mathrm{cm}^{-3}\right)\end{array}$ & $\begin{array}{c}\sigma_{\rho} \\
\left(\mathrm{g} \mathrm{cm}^{-3}\right)\end{array}$ \\
\hline 362 & Havnia & MMB & $\mathrm{Ch}$ & $1.67 \times 10^{17}$ & $8.36 \times 10^{16}$ & 89.09 & 3.97 & 0.45 & 0.23 \\
\hline 366 & Vincentina & OMB & $\mathrm{Ch}$ & $1.00 \times 10^{17}$ & $5.00 \times 10^{16}$ & 87.73 & 3.54 & 0.28 & 0.15 \\
\hline 373 & Melusina & OMB & $\mathrm{Ch}$ & $3.43 \times 10^{17}$ & $1.72 \times 10^{17}$ & 96.76 & 2.85 & 0.72 & 0.37 \\
\hline 377 & Campania & MMB & $\mathrm{Ch}$ & $1.52 \times 10^{17}$ & $7.61 \times 10^{16}$ & 92.40 & 1.08 & 0.37 & 0.18 \\
\hline 404 & Arsinoe & MMB & $\mathrm{Ch}$ & $8.21 \times 10^{17}$ & $1.70 \times 10^{17}$ & 95.78 & 3.10 & 1.78 & 0.41 \\
\hline 405 & Thia & MMB & $\mathrm{Ch}$ & $1.68 \times 10^{18}$ & $6.79 \times 10^{17}$ & 119.36 & 7.70 & 1.89 & 0.85 \\
\hline 407 & Arachne & MMB & $\mathrm{Ch}$ & $1.26 \times 10^{17}$ & $6.28 \times 10^{16}$ & 97.33 & 1.16 & 0.26 & 0.13 \\
\hline 410 & Chloris & MMB & $\mathrm{Ch}$ & $1.89 \times 10^{18}$ & $5.95 \times 10^{17}$ & 111.18 & 7.35 & 2.63 & 0.98 \\
\hline 442 & Eichsfeldia & IMB & $\mathrm{Ch}$ & $2.50 \times 10^{17}$ & $1.23 \times 10^{17}$ & 65.30 & 1.65 & 1.71 & 0.85 \\
\hline 445 & Edna & OMB & $\mathrm{Ch}$ & $1.24 \times 10^{18}$ & $6.10 \times 10^{17}$ & 88.11 & 2.18 & 3.46 & 1.72 \\
\hline 481 & Emita & MMB & $\mathrm{Ch}$ & $1.31 \times 10^{18}$ & $6.24 \times 10^{17}$ & 107.74 & 5.40 & 2.00 & 1.00 \\
\hline 488 & Kreusa & OMB & $\mathrm{Ch}$ & $2.47 \times 10^{18}$ & $4.25 \times 10^{17}$ & 167.51 & 6.56 & 1.00 & 0.21 \\
\hline 490 & Veritas & OMB & $\mathrm{Ch}$ & $2.02 \times 10^{18}$ & $9.87 \times 10^{17}$ & 114.87 & 3.64 & 2.55 & 1.27 \\
\hline 503 & Evelyn & MMB & $\mathrm{Ch}$ & $8.28 \times 10^{17}$ & $3.85 \times 10^{17}$ & 89.48 & 2.49 & 2.21 & 1.04 \\
\hline 521 & Brixia & MMB & $\mathrm{Ch}$ & $4.10 \times 10^{17}$ & $2.09 \times 10^{17}$ & 118.11 & 6.86 & 0.47 & 0.26 \\
\hline 554 & Peraga & IMB & $\mathrm{Ch}$ & $5.86 \times 10^{17}$ & $2.18 \times 10^{17}$ & 96.65 & 1.19 & 1.24 & 0.46 \\
\hline 602 & Marianna & OMB & $\mathrm{Ch}$ & $3.20 \times 10^{18}$ & $1.36 \times 10^{18}$ & 127.54 & 2.55 & 2.95 & 1.26 \\
\hline 654 & Zelinda & IMB & $\mathrm{Ch}$ & $1.22 \times 10^{18}$ & $3.51 \times 10^{17}$ & 125.90 & 4.49 & 1.17 & 0.36 \\
\hline 694 & Ekard & MMB & $\mathrm{Ch}$ & $1.20 \times 10^{17}$ & $5.94 \times 10^{16}$ & 93.95 & 4.82 & 0.28 & 0.14 \\
\hline 735 & Marghanna & MMB & $\mathrm{Ch}$ & $7.23 \times 10^{17}$ & $2.98 \times 10^{17}$ & 71.75 & 2.11 & 3.74 & 1.58 \\
\hline 751 & Faina & MMB & $\mathrm{Ch}$ & $3.67 \times 10^{18}$ & $6.38 \times 10^{17}$ & 106.86 & 1.15 & 5.74 & 1.02 \\
\hline 776 & Berbericia & OMB & $\mathrm{Cgh}$ & $4.46 \times 10^{18}$ & $4.16 \times 10^{18}$ & 150.92 & 3.20 & 2.48 & 2.31 \\
\hline 788 & Hohensteina & OMB & $\mathrm{Ch}$ & $1.85 \times 10^{17}$ & $9.22 \times 10^{16}$ & 114.68 & 6.38 & 0.23 & 0.12 \\
\hline 791 & Ani & OMB & $\mathrm{Ch}$ & $1.47 \times 10^{17}$ & $7.37 \times 10^{16}$ & 99.20 & 4.96 & 0.29 & 0.15 \\
\hline 914 & Palisana & IMB & $\mathrm{Ch}$ & $4.89 \times 10^{17}$ & $2.29 \times 10^{17}$ & 78.71 & 2.64 & 1.92 & 0.92 \\
\hline 1467 & Mashona & Cybele & $\mathrm{Ch}$ & $2.04 \times 10^{17}$ & $1.02 \times 10^{17}$ & 95.08 & 1.30 & 0.45 & 0.23 \\
\hline
\end{tabular}


Table D.2. The diameter estimates $(\mathcal{D})$ for all the $\mathrm{Ch} / \mathrm{Cgh}$ asteroids available in the literature.

\begin{tabular}{|c|c|c|c|c|c|}
\hline \# & Name & $\begin{array}{c}\mathcal{D} \\
(\mathrm{km})\end{array}$ & $\begin{array}{c}\delta \mathcal{D} \\
(\mathrm{km})\end{array}$ & Method & Reference \\
\hline 13 & Egeria & 244.0 & 73.2 & STM & Morrison \& Zellner (2007) \\
\hline 13 & Egeria & 207.6 & 24.9 & STM & Tedesco et al. (2004b) \\
\hline 13 & Egeria & 203.1 & 5.9 & OCC & Dunham et al. (2017) \\
\hline 13 & Egeria & 223.1 & 10.4 & STM & Ryan \& Woodward (2010) \\
\hline 13 & Egeria & 226.1 & 28.5 & NEATM & Ryan \& Woodward (2010) \\
\hline 13 & Egeria & 203.4 & 7.7 & STM & Usui et al. (2011) \\
\hline 13 & Egeria & 227.0 & 77.9 & NEATM & Masiero et al. (2011) \\
\hline 13 & Egeria & 202.6 & 150.2 & NEATM & Nugent et al. (2015) \\
\hline 13 & Egeria & 209.0 & 24.0 & ADAM & Hanuš et al. (2017b) \\
\hline 13 & Egeria & 201.0 & 12.0 & ADAM & Hanuš et al. (2017b) \\
\hline 19 & Fortuna & 221.0 & 66.3 & STM & Morrison \& Zellner (2007) \\
\hline 19 & Fortuna & 210.1 & 11.3 & OCC & Dunham et al. (2017) \\
\hline 19 & Fortuna & 209.6 & 14.9 & IM-TE & Drummond et al. (2011) \\
\hline 19 & Fortuna & 199.7 & 9.1 & TPM & Usui et al. (2011) \\
\hline 19 & Fortuna & 223.0 & 130.8 & NEATM & Masiero et al. (2011) \\
\hline 19 & Fortuna & 209.8 & 6.6 & NEATM & Masiero et al. (2012) \\
\hline 19 & Fortuna & 187.0 & 39.0 & LCIMG & Hanuš et al. (2013b) \\
\hline 19 & Fortuna & 211.0 & 12.0 & ADAM & Hanuš et al. (2017b) \\
\hline 34 & Circe & 111.0 & 33.3 & STM & Morrison \& Zellner (2007) \\
\hline 34 & Circe & 113.5 & 9.9 & STM & Tedesco et al. (2004b) \\
\hline 34 & Circe & 109.5 & 5.4 & OCC & Dunham et al. (2017) \\
\hline 34 & Circe & 97.4 & 8.5 & STM & Ryan \& Woodward (2010) \\
\hline 34 & Circe & 121.5 & 21.3 & NEATM & Ryan \& Woodward (2010) \\
\hline 34 & Circe & 96.0 & 30.0 & LCOCC & Ďurech et al. (2011) \\
\hline 34 & Circe & 107.0 & 30.0 & LCOCC & Ďurech et al. (2011) \\
\hline 34 & Circe & 116.5 & 3.4 & STM & Usui et al. (2011) \\
\hline 34 & Circe & 113.2 & 8.7 & NEATM & Masiero et al. (2011) \\
\hline 34 & Circe & 133.0 & 3.1 & NEATM & Masiero et al. (2012) \\
\hline 34 & Circe & 117.0 & 42.0 & LCIMG & Hanuš et al. (2013b) \\
\hline 34 & Circe & 116.0 & 33.0 & LCIMG & Hanuš et al. (2013b) \\
\hline 34 & Circe & 114.1 & 131.3 & NEATM & Nugent et al. (2015) \\
\hline 38 & Leda & 115.9 & 6.3 & STM & Tedesco et al. (2004b) \\
\hline 38 & Leda & 97.3 & 8.1 & STM & Ryan \& Woodward (2010) \\
\hline 38 & Leda & 118.1 & 16.0 & NEATM & Ryan \& Woodward (2010) \\
\hline 38 & Leda & 114.2 & 4.6 & STM & Usui et al. (2011) \\
\hline 38 & Leda & 116.0 & 46.5 & NEATM & Masiero et al. (2011) \\
\hline 38 & Leda & 122.5 & 3.7 & NEATM & Masiero et al. (2012) \\
\hline 38 & Leda & 114.2 & 84.6 & NEATM & Nugent et al. (2015) \\
\hline 41 & Daphne & 203.0 & 60.9 & STM & Morrison \& Zellner (2007) \\
\hline 41 & Daphne & 174.0 & 35.1 & STM & Tedesco et al. (2004b) \\
\hline 41 & Daphne & 172.4 & 12.2 & STM & Ryan \& Woodward (2010) \\
\hline 41 & Daphne & 207.9 & 31.6 & NEATM & Ryan \& Woodward (2010) \\
\hline 41 & Daphne & 187.0 & 60.0 & LCOCC & Ďurech et al. (2011) \\
\hline 41 & Daphne & 201.5 & 22.5 & TPM & Matter et al. (2011) \\
\hline 41 & Daphne & 185.5 & 10.5 & TPM & Matter et al. (2011) \\
\hline 41 & Daphne & 179.6 & 7.7 & STM & Usui et al. (2011) \\
\hline 41 & Daphne & 205.5 & 5.6 & NEATM & Masiero et al. (2012) \\
\hline 41 & Daphne & 186.0 & 81.0 & LCIMG & Hanuš et al. (2013b) \\
\hline 41 & Daphne & 198.7 & 185.1 & NEATM & Nugent et al. (2015) \\
\hline 41 & Daphne & 188.0 & 15.0 & ADAM & Hanuš et al. (2017b) \\
\hline 41 & Daphne & 187.0 & 27.9 & KOALA & This work \\
\hline 48 & Doris & 221.8 & 22.5 & STM & Tedesco et al. (2004b) \\
\hline 48 & Doris & 196.3 & 47.3 & OCC & Dunham et al. (2017) \\
\hline 48 & Doris & 211.3 & 48.0 & STM & Ryan \& Woodward (2010) \\
\hline 48 & Doris & 238.8 & 27.6 & NEATM & Ryan \& Woodward (2010) \\
\hline
\end{tabular}

Notes. For each, the $3 \sigma$ uncertainty, method, and bibliographic reference are reported. The methods are IM-TE: Ellipsoid from Imaging, LCIMG: 3D Model scaled with Imaging, ADAM \& KOALA: Multidata 3D Modeling, OCC: Stellar Occultations, STM: Standard Thermal Model, NEATM: Near-Earth Asteroid Thermal Model, and TPM: Thermophysical Model. 
B. Carry et al.: Homogeneous internal structure of CM-like asteroid (41) Daphne

Table D.2. continued.

\begin{tabular}{|c|c|c|c|c|c|}
\hline$\#$ & Name & $\begin{array}{c}\mathcal{D} \\
(\mathrm{km})\end{array}$ & $\begin{array}{c}\delta \mathcal{D} \\
(\mathrm{km})\end{array}$ & Method & Reference \\
\hline 48 & Doris & 200.3 & 8.2 & STM & Usui et al. (2011) \\
\hline 48 & Doris & 223.4 & 12.5 & NEATM & Masiero et al. (2011) \\
\hline 48 & Doris & 165.4 & 125.4 & NEATM & Nugent et al. (2015) \\
\hline 49 & Pales & 149.8 & 11.4 & STM & Tedesco et al. (2004b) \\
\hline 49 & Pales & 157.5 & 14.7 & STM & Ryan \& Woodward (2010) \\
\hline 49 & Pales & 169.7 & 27.9 & NEATM & Ryan \& Woodward (2010) \\
\hline 49 & Pales & 148.0 & 7.7 & STM & Usui et al. (2011) \\
\hline 49 & Pales & 166.2 & 6.5 & NEATM & Masiero et al. (2012) \\
\hline 49 & Pales & 149.3 & 142.6 & NEATM & Nugent et al. (2015) \\
\hline 49 & Pales & 138.8 & 124.6 & NEATM & Nugent et al. (2015) \\
\hline 50 & Virginia & 99.8 & 15.6 & STM & Tedesco et al. (2004b) \\
\hline 50 & Virginia & 99.0 & 9.1 & NEATM & Ryan \& Woodward (2010) \\
\hline 50 & Virginia & 84.4 & 2.5 & STM & Usui et al. (2011) \\
\hline 50 & Virginia & 100.0 & 22.8 & NEATM & Masiero et al. (2011) \\
\hline 50 & Virginia & 87.0 & 2.8 & NEATM & Masiero et al. (2012) \\
\hline 51 & Nemausa & 151.0 & 45.3 & STM & Morrison \& Zellner (2007) \\
\hline 51 & Nemausa & 147.9 & 7.2 & STM & Tedesco et al. (2004b) \\
\hline 51 & Nemausa & 155.9 & 11.9 & STM & Ryan \& Woodward (2010) \\
\hline 51 & Nemausa & 147.2 & 5.1 & STM & Usui et al. (2011) \\
\hline 51 & Nemausa & 142.6 & 37.5 & NEATM & Masiero et al. (2011) \\
\hline 51 & Nemausa & 146.1 & 248.9 & NEATM & Masiero et al. (2012) \\
\hline 51 & Nemausa & 144.0 & 9.0 & ADAM & Hanuš et al. (2017b) \\
\hline 54 & Alexandra & 175.0 & 52.5 & STM & Morrison \& Zellner (2007) \\
\hline 54 & Alexandra & 165.8 & 10.2 & STM & Tedesco et al. (2004b) \\
\hline 54 & Alexandra & 147.0 & 7.0 & OCC & Dunham et al. (2017) \\
\hline 54 & Alexandra & 177.4 & 13.7 & STM & Ryan \& Woodward (2010) \\
\hline 54 & Alexandra & 177.7 & 22.7 & NEATM & Ryan \& Woodward (2010) \\
\hline 54 & Alexandra & 135.0 & 60.0 & LCOCC & Durech et al. (2011) \\
\hline 54 & Alexandra & 142.0 & 27.0 & LCOCC & Ďurech et al. (2011) \\
\hline 54 & Alexandra & 144.5 & 5.4 & STM & Usui et al. (2011) \\
\hline 54 & Alexandra & 142.0 & 44.3 & NEATM & Masiero et al. (2011) \\
\hline 54 & Alexandra & 160.1 & 5.6 & NEATM & Masiero et al. (2012) \\
\hline 54 & Alexandra & 128.0 & 33.0 & LCIMG & Hanuš et al. (2013b) \\
\hline 54 & Alexandra & 143.0 & 15.0 & ADAM & Hanuš et al. (2017b) \\
\hline 58 & Concordia & 93.4 & 9.0 & STM & Tedesco et al. (2004b) \\
\hline 58 & Concordia & 93.6 & 3.2 & NEATM & Usui et al. (2011) \\
\hline 58 & Concordia & 94.6 & 9.3 & STM & Ryan \& Woodward (2010) \\
\hline 58 & Concordia & 102.7 & 23.3 & NEATM & Ryan \& Woodward (2010) \\
\hline 58 & Concordia & 92.3 & 4.6 & NEATM & Masiero et al. (2011) \\
\hline 58 & Concordia & 106.5 & 2.2 & NEATM & Masiero et al. (2012) \\
\hline 58 & Concordia & 88.4 & 72.8 & NEATM & Nugent et al. (2015) \\
\hline 58 & Concordia & 95.8 & 3.9 & OCC & Dunham et al. (2017) \\
\hline 58 & Concordia & 96.0 & 2.5 & OCC & Dunham et al. (2017) \\
\hline 62 & Erato & 95.4 & 6.0 & STM & Tedesco et al. (2004b) \\
\hline 62 & Erato & 78.6 & 2.7 & NEATM & Usui et al. (2011) \\
\hline 62 & Erato & 80.7 & 6.3 & STM & Ryan \& Woodward (2010) \\
\hline 62 & Erato & 105.6 & 16.1 & NEATM & Ryan \& Woodward (2010) \\
\hline 62 & Erato & 106.9 & 2.0 & NEATM & Masiero et al. (2012) \\
\hline 62 & Erato & 82.3 & 71.1 & NEATM & Nugent et al. (2015) \\
\hline 62 & Erato & 59.4 & 77.1 & NEATM & Nugent et al. (2015) \\
\hline 70 & Panopaea & 151.0 & 45.3 & STM & Morrison \& Zellner (2007) \\
\hline 70 & Panopaea & 122.2 & 6.9 & STM & Tedesco et al. (2004b) \\
\hline 70 & Panopaea & 105.2 & 8.4 & STM & Ryan \& Woodward (2010) \\
\hline 70 & Panopaea & 130.9 & 19.6 & NEATM & Ryan \& Woodward (2010) \\
\hline 70 & Panopaea & 131.2 & 4.5 & OCC & Dunham et al. (2017) \\
\hline 70 & Panopaea & 141.4 & 5.7 & STM & Usui et al. (2011) \\
\hline 70 & Panopaea & 139.0 & 11.5 & NEATM & Masiero et al. (2011) \\
\hline 70 & Panopaea & 162.6 & 3.8 & NEATM & Masiero et al. (2012) \\
\hline
\end{tabular}


Table D.2. continued.

\begin{tabular}{|c|c|c|c|c|c|}
\hline$\#$ & Name & $\begin{array}{c}\mathcal{D} \\
(\mathrm{km})\end{array}$ & $\begin{array}{c}\delta \mathcal{D} \\
(\mathrm{km})\end{array}$ & Method & Reference \\
\hline 70 & Panopaea & 115.3 & 127.5 & NEATM & Nugent et al. (2015) \\
\hline 78 & Diana & 120.6 & 8.1 & STM & Tedesco et al. (2004b) \\
\hline 78 & Diana & 131.2 & 10.5 & OCC & Dunham et al. (2017) \\
\hline 78 & Diana & 116.0 & 14.5 & STM & Ryan \& Woodward (2010) \\
\hline 78 & Diana & 130.8 & 22.0 & NEATM & Ryan \& Woodward (2010) \\
\hline 78 & Diana & 126.5 & 5.0 & STM & Usui et al. (2011) \\
\hline 78 & Diana & 179.3 & 146.2 & NEATM & Masiero et al. (2012) \\
\hline 78 & Diana & 160.1 & 137.1 & NEATM & Nugent et al. (2015) \\
\hline 84 & Klio & 87.0 & 26.1 & STM & Morrison \& Zellner (2007) \\
\hline 84 & Klio & 79.2 & 4.8 & STM & Tedesco et al. (2004b) \\
\hline 84 & Klio & 68.1 & 6.5 & STM & Ryan \& Woodward (2010) \\
\hline 84 & Klio & 81.1 & 10.3 & NEATM & Ryan \& Woodward (2010) \\
\hline 84 & Klio & 78.3 & 2.9 & STM & Usui et al. (2011) \\
\hline 84 & Klio & 79.0 & 14.6 & NEATM & Masiero et al. (2011) \\
\hline 91 & Aegina & 104.0 & 31.2 & STM & Morrison \& Zellner (2007) \\
\hline 91 & Aegina & 109.8 & 9.9 & STM & Tedesco et al. (2004b) \\
\hline 91 & Aegina & 111.6 & 6.3 & OCC & Dunham et al. (2017) \\
\hline 91 & Aegina & 100.2 & 3.7 & NEATM & Usui et al. (2011) \\
\hline 91 & Aegina & 123.1 & 38.7 & NEATM & Ryan \& Woodward (2010) \\
\hline 91 & Aegina & 104.7 & 9.3 & NEATM & Masiero et al. (2011) \\
\hline 91 & Aegina & 102.7 & 5.7 & NEATM & Masiero et al. (2011) \\
\hline 91 & Aegina & 98.4 & 111.5 & NEATM & Nugent et al. (2015) \\
\hline 95 & Arethusa & 229.0 & 68.7 & STM & Morrison \& Zellner (2007) \\
\hline 95 & Arethusa & 136.0 & 30.3 & STM & Tedesco et al. (2004b) \\
\hline 95 & Arethusa & 131.1 & 8.7 & OCC & Dunham et al. (2017) \\
\hline 95 & Arethusa & 157.9 & 10.2 & OCC & Dunham et al. (2017) \\
\hline 95 & Arethusa & 135.1 & 4.8 & OCC & Dunham et al. (2017) \\
\hline 95 & Arethusa & 118.3 & 14.1 & STM & Ryan \& Woodward (2010) \\
\hline 95 & Arethusa & 139.5 & 21.3 & NEATM & Ryan \& Woodward (2010) \\
\hline 95 & Arethusa & 143.8 & 14.7 & STM & Usui et al. (2011) \\
\hline 95 & Arethusa & 152.4 & 15.3 & STM & Masiero et al. (2011) \\
\hline 95 & Arethusa & 147.9 & 14.7 & STM & Masiero et al. (2011) \\
\hline 95 & Arethusa & 133.4 & 118.4 & NEATM & Nugent et al. (2015) \\
\hline 98 & Ianthe & 104.4 & 5.4 & STM & Tedesco et al. (2004b) \\
\hline 98 & Ianthe & 102.0 & 11.0 & STM & Ryan \& Woodward (2010) \\
\hline 98 & Ianthe & 114.3 & 15.7 & NEATM & Ryan \& Woodward (2010) \\
\hline 98 & Ianthe & 104.2 & 3.9 & STM & Usui et al. (2011) \\
\hline 98 & Ianthe & 110.9 & 7.0 & NEATM & Masiero et al. (2011) \\
\hline 98 & Ianthe & 132.8 & 3.4 & NEATM & Masiero et al. (2012) \\
\hline 104 & Klymene & 123.7 & 9.3 & STM & Tedesco et al. (2004b) \\
\hline 104 & Klymene & 109.9 & 11.5 & STM & Ryan \& Woodward (2010) \\
\hline 104 & Klymene & 131.2 & 19.9 & NEATM & Ryan \& Woodward (2010) \\
\hline 104 & Klymene & 126.5 & 5.6 & STM & Usui et al. (2011) \\
\hline 104 & Klymene & 125.8 & 7.9 & STM & Masiero et al. (2011) \\
\hline 104 & Klymene & 136.6 & 4.6 & STM & Masiero et al. (2012) \\
\hline 104 & Klymene & 124.6 & 110.9 & NEATM & Nugent et al. (2015) \\
\hline 105 & Artemis & 126.0 & 37.8 & STM & Morrison \& Zellner (2007) \\
\hline 105 & Artemis & 103.7 & 15.8 & STM & Tedesco et al. (2004b) \\
\hline 105 & Artemis & 119.1 & 8.4 & STM & Ryan \& Woodward (2010) \\
\hline 105 & Artemis & 101.1 & 8.4 & NEATM & Ryan \& Woodward (2010) \\
\hline 105 & Artemis & 123.5 & 4.5 & STM & Usui et al. (2011) \\
\hline 105 & Artemis & 119.0 & 52.0 & NEATM & Masiero et al. (2011) \\
\hline 105 & Artemis & 112.8 & 102.1 & NEATM & Masiero et al. (2012) \\
\hline 105 & Artemis & 112.8 & 105.7 & NEATM & Nugent et al. (2015) \\
\hline 105 & Artemis & 94.9 & 69.7 & NEATM & Nugent et al. (2015) \\
\hline 106 & Dione & 140.0 & 42.0 & STM & Morrison \& Zellner (2007) \\
\hline 106 & Dione & 145.6 & 2.5 & OCC & Dunham et al. (2017) \\
\hline
\end{tabular}


B. Carry et al.: Homogeneous internal structure of CM-like asteroid (41) Daphne

Table D.2. continued.

\begin{tabular}{|c|c|c|c|c|c|}
\hline$\#$ & Name & $\begin{array}{c}\mathcal{D} \\
(\mathrm{km})\end{array}$ & $\begin{array}{c}\delta \mathcal{D} \\
(\mathrm{km})\end{array}$ & Method & Reference \\
\hline 106 & Dione & 146.6 & 8.4 & STM & Tedesco et al. (2004b) \\
\hline 106 & Dione & 127.3 & 10.1 & STM & Ryan \& Woodward (2010) \\
\hline 106 & Dione & 162.9 & 23.6 & NEATM & Ryan \& Woodward (2010) \\
\hline 106 & Dione & 153.4 & 7.1 & STM & Usui et al. (2011) \\
\hline 106 & Dione & 207.9 & 6.5 & NEATM & Masiero et al. (2012) \\
\hline 106 & Dione & 83.4 & 2.5 & NEATM & Grav et al. (2012) \\
\hline 106 & Dione & 89.2 & 101.9 & NEATM & Nugent et al. (2015) \\
\hline 106 & Dione & 138.8 & 137.5 & NEATM & Nugent et al. (2015) \\
\hline 106 & Dione & 151.7 & 77.7 & NEATM & Ryan et al. (2015) \\
\hline 106 & Dione & 168.9 & 86.4 & NEATM & Ryan et al. (2015) \\
\hline 106 & Dione & 182.9 & 93.5 & NEATM & Ryan et al. (2015) \\
\hline 109 & Felicitas & 75.0 & 22.5 & STM & Morrison \& Zellner (2007) \\
\hline 109 & Felicitas & 89.4 & 7.5 & STM & Tedesco et al. (2004b) \\
\hline 109 & Felicitas & 88.2 & 2.4 & OCC & Dunham et al. (2017) \\
\hline 109 & Felicitas & 79.5 & 7.3 & STM & Ryan \& Woodward (2010) \\
\hline 109 & Felicitas & 111.4 & 27.4 & NEATM & Ryan \& Woodward (2010) \\
\hline 109 & Felicitas & 80.8 & 3.7 & STM & Usui et al. (2011) \\
\hline 109 & Felicitas & 89.0 & 18.5 & STM & Masiero et al. (2011) \\
\hline 109 & Felicitas & 99.9 & 3.0 & STM & Masiero et al. (2012) \\
\hline 109 & Felicitas & 67.5 & 2.0 & NEATM & Grav et al. (2012) \\
\hline 109 & Felicitas & 92.4 & 94.6 & NEATM & Nugent et al. (2015) \\
\hline 109 & Felicitas & 64.7 & 71.5 & NEATM & Nugent et al. (2015) \\
\hline 109 & Felicitas & 94.6 & 85.2 & NEATM & Nugent et al. (2015) \\
\hline 111 & Ate & 134.6 & 13.8 & STM & Tedesco et al. (2004b) \\
\hline 111 & Ate & 119.0 & 19.2 & OCC & Dunham et al. (2017) \\
\hline 111 & Ate & 130.4 & 2.7 & OCC & Dunham et al. (2017) \\
\hline 111 & Ate & 134.9 & 1.2 & OCC & Dunham et al. (2017) \\
\hline 111 & Ate & 140.2 & 7.2 & STM & Ryan \& Woodward (2010) \\
\hline 111 & Ate & 153.2 & 17.4 & NEATM & Ryan \& Woodward (2010) \\
\hline 111 & Ate & 146.6 & 7.0 & STM & Usui et al. (2011) \\
\hline 111 & Ate & 135.0 & 55.7 & NEATM & Masiero et al. (2011) \\
\hline 112 & Iphigenia & 72.2 & 13.2 & STM & Tedesco et al. (2004b) \\
\hline 112 & Iphigenia & 59.8 & 6.2 & STM & Ryan \& Woodward (2010) \\
\hline 112 & Iphigenia & 80.6 & 12.7 & NEATM & Ryan \& Woodward (2010) \\
\hline 112 & Iphigenia & 71.1 & 2.8 & STM & Usui et al. (2011) \\
\hline 112 & Iphigenia & 70.4 & 8.7 & NEATM & Masiero et al. (2011) \\
\hline 112 & Iphigenia & 84.9 & 68.6 & NEATM & Masiero et al. (2012) \\
\hline 112 & Iphigenia & 69.6 & 64.6 & NEATM & Nugent et al. (2015) \\
\hline 112 & Iphigenia & 70.3 & 52.9 & NEATM & Nugent et al. (2015) \\
\hline 121 & Hermione & 209.0 & 14.1 & STM & Tedesco et al. (2004b) \\
\hline 121 & Hermione & 178.9 & 21.6 & IM & Marchis et al. (2005) \\
\hline 121 & Hermione & 138.8 & 35.7 & $\mathrm{IM}$ & Marchis et al. (2005) \\
\hline 121 & Hermione & 189.0 & 21.0 & $\mathrm{IM}$ & Marchis et al. (2006) \\
\hline 121 & Hermione & 187.0 & 18.0 & KOALA & Descamps et al. (2009) \\
\hline 121 & Hermione & 221.6 & 17.9 & STM & Ryan \& Woodward (2010) \\
\hline 121 & Hermione & 212.0 & 23.1 & NEATM & Ryan \& Woodward (2010) \\
\hline 121 & Hermione & 194.1 & 8.1 & STM & Usui et al. (2011) \\
\hline 121 & Hermione & 165.0 & 13.5 & NEATM & Masiero et al. (2011) \\
\hline 121 & Hermione & 192.4 & 22.1 & NEATM & Marchis et al. (2012) \\
\hline 121 & Hermione & 220.0 & 66.0 & TPM & Marchis et al. (2012) \\
\hline 121 & Hermione & 201.1 & 190.3 & NEATM & Nugent et al. (2015) \\
\hline 121 & Hermione & 155.3 & 158.8 & NEATM & Nugent et al. (2015) \\
\hline 127 & Johanna & 123.3 & 13.1 & STM & Tedesco et al. (2004a) \\
\hline 127 & Johanna & 113.0 & 26.4 & OCC & Dunham et al. (2017) \\
\hline 127 & Johanna & 109.6 & 0.0 & STM & Ryan \& Woodward (2010) \\
\hline 127 & Johanna & 120.0 & 0.0 & NEATM & Ryan \& Woodward (2010) \\
\hline 127 & Johanna & 114.2 & 4.6 & TPM & Usui et al. (2011) \\
\hline 127 & Johanna & 129.1 & 3.0 & NEATM & Masiero et al. (2012) \\
\hline
\end{tabular}


Table D.2. continued.

\begin{tabular}{|c|c|c|c|c|c|}
\hline$\#$ & Name & $\begin{array}{c}\mathcal{D} \\
(\mathrm{km})\end{array}$ & $\begin{array}{c}\delta \mathcal{D} \\
(\mathrm{km})\end{array}$ & Method & Reference \\
\hline 127 & Johanna & 108.0 & 30.0 & LCOCC & Marciniak et al. (2012) \\
\hline 127 & Johanna & 116.0 & 30.0 & LCOCC & Marciniak et al. (2012) \\
\hline 127 & Johanna & 106.4 & 127.9 & NEATM & Nugent et al. (2015) \\
\hline 130 & Elektra & 174.0 & 52.2 & STM & Morrison \& Zellner (2007) \\
\hline 130 & Elektra & 182.2 & 35.4 & STM & Tedesco et al. (2004b) \\
\hline 130 & Elektra & 191.0 & 6.0 & IM & Marchis et al. (2006) \\
\hline 130 & Elektra & 196.0 & 33.0 & NEATM & Marchis et al. (2008b) \\
\hline 130 & Elektra & 215.0 & 45.0 & IM & Marchis et al. (2008b) \\
\hline 130 & Elektra & 158.8 & 18.3 & STM & Ryan \& Woodward (2010) \\
\hline 130 & Elektra & 200.5 & 40.0 & NEATM & Ryan \& Woodward (2010) \\
\hline 130 & Elektra & 191.0 & 42.0 & LCOCC & Durech et al. (2011) \\
\hline 130 & Elektra & 183.0 & 6.8 & STM & Usui et al. (2011) \\
\hline 130 & Elektra & 198.9 & 12.3 & NEATM & Masiero et al. (2011) \\
\hline 130 & Elektra & 161.9 & 11.5 & NEATM & Masiero et al. (2012) \\
\hline 130 & Elektra & 201.2 & 25.5 & NEATM & Marchis et al. (2012) \\
\hline 130 & Elektra & 197.0 & 60.0 & TPM & Marchis et al. (2012) \\
\hline 130 & Elektra & 185.0 & 60.0 & LCIMG & Hanuš et al. (2013b) \\
\hline 130 & Elektra & 158.9 & 138.0 & NEATM & Nugent et al. (2015) \\
\hline 130 & Elektra & 199.0 & 21.0 & ADAM & Hanuš et al. (2017a) \\
\hline 134 & Sophrosyne & 123.3 & 6.0 & STM & Tedesco et al. (2004b) \\
\hline 134 & Sophrosyne & 186.1 & 57.0 & OCC & Dunham et al. (2017) \\
\hline 134 & Sophrosyne & 122.9 & 14.7 & STM & Ryan \& Woodward (2010) \\
\hline 134 & Sophrosyne & 127.2 & 18.7 & NEATM & Ryan \& Woodward (2010) \\
\hline 134 & Sophrosyne & 100.4 & 4.0 & STM & Usui et al. (2011) \\
\hline 134 & Sophrosyne & 112.2 & 32.4 & STM & Masiero et al. (2011) \\
\hline 134 & Sophrosyne & 104.5 & 3.8 & STM & Masiero et al. (2012) \\
\hline 141 & Lumen & 120.4 & 13.7 & STM & Tedesco et al. (2004a) \\
\hline 141 & Lumen & 131.0 & 8.7 & STM & Tedesco et al. (2004b) \\
\hline 141 & Lumen & 137.4 & 36.2 & OCC & Dunham et al. (2017) \\
\hline 141 & Lumen & 110.9 & 9.8 & STM & Ryan \& Woodward (2010) \\
\hline 141 & Lumen & 139.8 & 23.7 & NEATM & Ryan \& Woodward (2010) \\
\hline 141 & Lumen & 132.2 & 4.5 & STM & Usui et al. (2011) \\
\hline 141 & Lumen & 137.1 & 43.7 & NEATM & Masiero et al. (2011) \\
\hline 144 & Vibilia & 131.0 & 39.3 & STM & Morrison \& Zellner (2007) \\
\hline 144 & Vibilia & 142.4 & 7.8 & STM & Tedesco et al. (2004b) \\
\hline 144 & Vibilia & 142.5 & 15.0 & OCC & Dunham et al. (2017) \\
\hline 144 & Vibilia & 138.4 & 27.4 & STM & Ryan \& Woodward (2010) \\
\hline 144 & Vibilia & 161.2 & 27.1 & NEATM & Ryan \& Woodward (2010) \\
\hline 144 & Vibilia & 142.2 & 5.3 & STM & Usui et al. (2011) \\
\hline 144 & Vibilia & 141.0 & 9.0 & ADAM & Hanuš et al. (2017b) \\
\hline 144 & Vibilia & 131.4 & 99.9 & NEATM & Nugent et al. (2015) \\
\hline 145 & Adeona & 151.1 & 9.6 & STM & Tedesco et al. (2004b) \\
\hline 145 & Adeona & 141.0 & 71.2 & OCC & Dunham et al. (2017) \\
\hline 145 & Adeona & 126.0 & 10.8 & STM & Ryan \& Woodward (2010) \\
\hline 145 & Adeona & 157.9 & 22.7 & NEATM & Ryan \& Woodward (2010) \\
\hline 145 & Adeona & 141.4 & 15.5 & STM & Usui et al. (2011) \\
\hline 145 & Adeona & 151.0 & 33.8 & NEATM & Masiero et al. (2011) \\
\hline 145 & Adeona & 151.0 & 25.7 & NEATM & Masiero et al. (2011) \\
\hline 145 & Adeona & 115.5 & 112.9 & NEATM & Nugent et al. (2015) \\
\hline 146 & Lucina & 132.2 & 7.2 & STM & Tedesco et al. (2004b) \\
\hline 146 & Lucina & 141.0 & 42.3 & STM & Morrison \& Zellner (2007) \\
\hline 146 & Lucina & 107.5 & 5.0 & OCC & Dunham et al. (2017) \\
\hline 146 & Lucina & 134.0 & 48.0 & OCC & Dunham et al. (2017) \\
\hline 146 & Lucina & 126.9 & 4.9 & NEATM & Usui et al. (2011) \\
\hline 146 & Lucina & 128.0 & 14.0 & STM & Ryan \& Woodward (2010) \\
\hline 146 & Lucina & 144.7 & 24.8 & NEATM & Ryan \& Woodward (2010) \\
\hline 146 & Lucina & 131.8 & 14.4 & NEATM & Masiero et al. (2011) \\
\hline 146 & Lucina & 160.3 & 3.6 & NEATM & Masiero et al. (2012) \\
\hline
\end{tabular}


B. Carry et al.: Homogeneous internal structure of CM-like asteroid (41) Daphne

Table D.2. continued.

\begin{tabular}{|c|c|c|c|c|c|}
\hline$\#$ & Name & $\begin{array}{c}\mathcal{D} \\
(\mathrm{km})\end{array}$ & $\begin{array}{c}\delta \mathcal{D} \\
(\mathrm{km})\end{array}$ & Method & Reference \\
\hline 146 & Lucina & 113.1 & 88.9 & NEATM & Nugent et al. (2015) \\
\hline 146 & Lucina & 119.0 & 33.0 & LCIMG & Hanuš et al. (2013b) \\
\hline 156 & Xanthippe & 121.0 & 7.5 & STM & Tedesco et al. (2004b) \\
\hline 156 & Xanthippe & 122.0 & 10.5 & STM & Ryan \& Woodward (2010) \\
\hline 156 & Xanthippe & 114.6 & 12.7 & NEATM & Ryan \& Woodward (2010) \\
\hline 156 & Xanthippe & 115.5 & 5.2 & STM & Usui et al. (2011) \\
\hline 156 & Xanthippe & 110.7 & 6.6 & NEATM & Masiero et al. (2011) \\
\hline 156 & Xanthippe & 143.4 & 2.7 & NEATM & Masiero et al. (2012) \\
\hline 156 & Xanthippe & 122.0 & 95.0 & NEATM & Nugent et al. (2015) \\
\hline 156 & Xanthippe & 112.4 & 116.6 & NEATM & Nugent et al. (2015) \\
\hline 159 & Aemilia & 140.0 & 42.0 & STM & Morrison \& Zellner (2007) \\
\hline 159 & Aemilia & 125.0 & 7.2 & STM & Tedesco et al. (2004b) \\
\hline 159 & Aemilia & 141.7 & 4.5 & OCC & Dunham et al. (2017) \\
\hline 159 & Aemilia & 123.3 & 15.3 & STM & Ryan \& Woodward (2010) \\
\hline 159 & Aemilia & 132.6 & 24.8 & NEATM & Ryan \& Woodward (2010) \\
\hline 159 & Aemilia & 130.0 & 6.9 & STM & Usui et al. (2011) \\
\hline 159 & Aemilia & 127.4 & 8.1 & STM & Masiero et al. (2011) \\
\hline 159 & Aemilia & 130.0 & 21.0 & LCOCC & Marciniak et al. (2018) \\
\hline 159 & Aemilia & 138.0 & 21.0 & LCOCC & Marciniak et al. (2018) \\
\hline 159 & Aemilia & 137.0 & 24.0 & LCTPM & Marciniak et al. (2018) \\
\hline 162 & Laurentia & 99.1 & 7.8 & STM & Tedesco et al. (2004b) \\
\hline 162 & Laurentia & 85.3 & 8.6 & NEATM & Usui et al. (2011) \\
\hline 162 & Laurentia & 102.4 & 12.2 & STM & Ryan \& Woodward (2010) \\
\hline 162 & Laurentia & 106.4 & 18.0 & NEATM & Ryan \& Woodward (2010) \\
\hline 162 & Laurentia & 104.0 & 9.8 & NEATM & Masiero et al. (2011) \\
\hline 162 & Laurentia & 101.3 & 3.2 & NEATM & Masiero et al. (2012) \\
\hline 162 & Laurentia & 97.7 & 1.7 & NEATM & Masiero et al. (2012) \\
\hline 162 & Laurentia & 97.2 & 11.1 & OCC & Dunham et al. (2017) \\
\hline 163 & Erigone & 72.6 & 17.1 & STM & Tedesco et al. (2004b) \\
\hline 163 & Erigone & 70.7 & 13.6 & STM & Ryan \& Woodward (2010) \\
\hline 163 & Erigone & 77.6 & 14.0 & NEATM & Ryan \& Woodward (2010) \\
\hline 163 & Erigone & 72.1 & 2.8 & STM & Usui et al. (2011) \\
\hline 163 & Erigone & 81.6 & 9.2 & NEATM & Masiero et al. (2011) \\
\hline 163 & Erigone & 69.7 & 59.8 & NEATM & Nugent et al. (2015) \\
\hline 168 & Sibylla & 148.4 & 12.0 & STM & Tedesco et al. (2004b) \\
\hline 168 & Sibylla & 154.6 & 6.0 & STM & Ryan \& Woodward (2010) \\
\hline 168 & Sibylla & 155.8 & 31.8 & NEATM & Ryan \& Woodward (2010) \\
\hline 168 & Sibylla & 146.5 & 5.2 & STM & Usui et al. (2011) \\
\hline 168 & Sibylla & 144.0 & 8.6 & NEATM & Masiero et al. (2011) \\
\hline 168 & Sibylla & 145.1 & 154.1 & NEATM & Nugent et al. (2015) \\
\hline 168 & Sibylla & 141.8 & 92.5 & NEATM & Nugent et al. (2015) \\
\hline 176 & Iduna & 121.0 & 6.6 & STM & Tedesco et al. (2004b) \\
\hline 176 & Iduna & 119.5 & 3.9 & NEATM & Usui et al. (2011) \\
\hline 176 & Iduna & 112.6 & 13.2 & STM & Ryan \& Woodward (2010) \\
\hline 176 & Iduna & 131.1 & 18.4 & NEATM & Ryan \& Woodward (2010) \\
\hline 176 & Iduna & 122.2 & 8.1 & NEATM & Masiero et al. (2011) \\
\hline 176 & Iduna & 115.1 & 127.8 & NEATM & Nugent et al. (2015) \\
\hline 176 & Iduna & 115.6 & 5.5 & OCC & Dunham et al. (2017) \\
\hline 176 & Iduna & 126.9 & 12.6 & OCC & Dunham et al. (2017) \\
\hline 187 & Lamberta & 130.4 & 8.1 & STM & Tedesco et al. (2004b) \\
\hline 187 & Lamberta & 131.4 & 14.0 & STM & Ryan \& Woodward (2010) \\
\hline 187 & Lamberta & 132.1 & 23.2 & NEATM & Ryan \& Woodward (2010) \\
\hline 187 & Lamberta & 130.4 & 5.7 & STM & Usui et al. (2011) \\
\hline 187 & Lamberta & 133.0 & 7.5 & NEATM & Masiero et al. (2011) \\
\hline 187 & Lamberta & 147.3 & 4.2 & NEATM & Masiero et al. (2012) \\
\hline 187 & Lamberta & 132.1 & 123.0 & NEATM & Nugent et al. (2015) \\
\hline 187 & Lamberta & 125.2 & 137.1 & NEATM & Nugent et al. (2015) \\
\hline 195 & Eurykleia & 85.7 & 5.1 & STM & Tedesco et al. (2004b) \\
\hline
\end{tabular}


Table D.2. continued.

\begin{tabular}{|c|c|c|c|c|c|}
\hline \# & Name & $\begin{array}{c}\mathcal{D} \\
(\mathrm{km})\end{array}$ & $\begin{array}{c}\delta \mathcal{D} \\
(\mathrm{km})\end{array}$ & Method & Reference \\
\hline 195 & Eurykleia & 89.4 & 3.3 & NEATM & Usui et al. (2011) \\
\hline 195 & Eurykleia & 82.9 & 10.0 & STM & Ryan \& Woodward (2010) \\
\hline 195 & Eurykleia & 88.3 & 12.2 & NEATM & Ryan \& Woodward (2010) \\
\hline 195 & Eurykleia & 80.3 & 6.0 & NEATM & Masiero et al. (2011) \\
\hline 195 & Eurykleia & 93.1 & 2.2 & NEATM & Masiero et al. (2012) \\
\hline 195 & Eurykleia & 75.0 & 56.8 & NEATM & Nugent et al. (2015) \\
\hline 195 & Eurykleia & 80.0 & 78.4 & NEATM & Nugent et al. (2015) \\
\hline 200 & Dynamene & 128.4 & 6.3 & STM & Tedesco et al. (2004b) \\
\hline 200 & Dynamene & 125.1 & 17.7 & STM & Ryan \& Woodward (2010) \\
\hline 200 & Dynamene & 135.9 & 20.7 & NEATM & Ryan \& Woodward (2010) \\
\hline 200 & Dynamene & 129.2 & 10.9 & OCC & Dunham et al. (2017) \\
\hline 200 & Dynamene & 133.8 & 5.2 & STM & Usui et al. (2011) \\
\hline 200 & Dynamene & 130.5 & 8.6 & NEATM & Masiero et al. (2011) \\
\hline 200 & Dynamene & 121.5 & 111.6 & NEATM & Nugent et al. (2015) \\
\hline 200 & Dynamene & 120.1 & 123.3 & NEATM & Nugent et al. (2015) \\
\hline 205 & Martha & 80.6 & 4.2 & STM & Tedesco et al. (2004b) \\
\hline 205 & Martha & 78.0 & 15.0 & STM & Tedesco et al. (2004a) \\
\hline 205 & Martha & 82.2 & 3.2 & NEATM & Usui et al. (2011) \\
\hline 205 & Martha & 68.5 & 5.6 & STM & Ryan \& Woodward (2010) \\
\hline 205 & Martha & 79.8 & 10.8 & NEATM & Ryan \& Woodward (2010) \\
\hline 205 & Martha & 65.5 & 1.6 & STM & Ryan \& Woodward (2010) \\
\hline 205 & Martha & 96.3 & 26.1 & NEATM & Ryan \& Woodward (2010) \\
\hline 205 & Martha & 81.5 & 2.3 & NEATM & Masiero et al. (2011) \\
\hline 205 & Martha & 93.2 & 2.2 & NEATM & Masiero et al. (2012) \\
\hline 205 & Martha & 71.4 & 59.3 & NEATM & Nugent et al. (2015) \\
\hline 205 & Martha & 64.4 & 11.7 & OCC & Dunham et al. (2017) \\
\hline 205 & Martha & 65.7 & 1.2 & OCC & Dunham et al. (2017) \\
\hline 211 & Isolda & 166.0 & 49.8 & STM & Morrison \& Zellner (2007) \\
\hline 211 & Isolda & 143.2 & 15.3 & STM & Tedesco et al. (2004b) \\
\hline 211 & Isolda & 142.6 & 13.1 & STM & Ryan \& Woodward (2010) \\
\hline 211 & Isolda & 150.9 & 22.5 & NEATM & Ryan \& Woodward (2010) \\
\hline 211 & Isolda & 153.5 & 5.1 & STM & Usui et al. (2011) \\
\hline 211 & Isolda & 143.0 & 64.9 & NEATM & Masiero et al. (2011) \\
\hline 211 & Isolda & 154.2 & 51.3 & NEATM & Masiero et al. (2012) \\
\hline 211 & Isolda & 142.5 & 144.2 & NEATM & Nugent et al. (2015) \\
\hline 238 & Hypatia & 154.0 & 46.2 & STM & Morrison \& Zellner (2007) \\
\hline 238 & Hypatia & 145.9 & 23.0 & OCC & Dunham et al. (2017) \\
\hline 238 & Hypatia & 148.5 & 10.8 & STM & Tedesco et al. (2004b) \\
\hline 238 & Hypatia & 149.2 & 42.5 & STM & Ryan \& Woodward (2010) \\
\hline 238 & Hypatia & 163.6 & 21.5 & NEATM & Ryan \& Woodward (2010) \\
\hline 238 & Hypatia & 144.0 & 4.6 & STM & Usui et al. (2011) \\
\hline 238 & Hypatia & 146.5 & 26.0 & NEATM & Masiero et al. (2011) \\
\hline 238 & Hypatia & 135.6 & 4.5 & NEATM & Masiero et al. (2012) \\
\hline 238 & Hypatia & 176.7 & 154.5 & NEATM & Nugent et al. (2015) \\
\hline 266 & Aline & 125.8 & 19.5 & STM & Tedesco et al. (2004a) \\
\hline 266 & Aline & 109.1 & 8.7 & STM & Tedesco et al. (2004b) \\
\hline 266 & Aline & 112.9 & 8.3 & STM & Ryan \& Woodward (2010) \\
\hline 266 & Aline & 125.2 & 25.2 & NEATM & Ryan \& Woodward (2010) \\
\hline 266 & Aline & 102.0 & 4.2 & STM & Usui et al. (2011) \\
\hline 266 & Aline & 109.0 & 55.0 & NEATM & Masiero et al. (2011) \\
\hline 266 & Aline & 152.5 & 190.2 & NEATM & Masiero et al. (2012) \\
\hline 266 & Aline & 89.4 & 79.0 & NEATM & Nugent et al. (2015) \\
\hline 303 & Josephina & 99.3 & 5.7 & STM & Tedesco et al. (2004b) \\
\hline 303 & Josephina & 98.7 & 5.2 & NEATM & Usui et al. (2011) \\
\hline 303 & Josephina & 105.4 & 1.6 & NEATM & Hasegawa et al. (2013) \\
\hline 303 & Josephina & 100.5 & 8.9 & STM & Ryan \& Woodward (2010) \\
\hline 303 & Josephina & 105.6 & 20.3 & NEATM & Ryan \& Woodward (2010) \\
\hline 303 & Josephina & 105.9 & 9.3 & NEATM & Masiero et al. (2011) \\
\hline
\end{tabular}


B. Carry et al.: Homogeneous internal structure of CM-like asteroid (41) Daphne

Table D.2. continued.

\begin{tabular}{|c|c|c|c|c|c|}
\hline \# & Name & $\begin{array}{c}\mathcal{D} \\
(\mathrm{km})\end{array}$ & $\begin{array}{c}\delta \mathcal{D} \\
(\mathrm{km})\end{array}$ & Method & Reference \\
\hline 303 & Josephina & 124.9 & 2.8 & NEATM & Masiero et al. (2012) \\
\hline 303 & Josephina & 97.6 & 0.8 & OCC & Dunham et al. (2017) \\
\hline 345 & Tercidina & 94.1 & 14.7 & STM & Tedesco et al. (2004b) \\
\hline 345 & Tercidina & 99.3 & 4.2 & OCC & Dunham et al. (2017) \\
\hline 345 & Tercidina & 93.8 & 17.7 & STM & Ryan \& Woodward (2010) \\
\hline 345 & Tercidina & 106.2 & 23.4 & NEATM & Ryan \& Woodward (2010) \\
\hline 345 & Tercidina & 99.2 & 3.0 & STM & Usui et al. (2011) \\
\hline 345 & Tercidina & 99.0 & 34.4 & NEATM & Masiero et al. (2011) \\
\hline 345 & Tercidina & 101.8 & 79.3 & NEATM & Masiero et al. (2012) \\
\hline 345 & Tercidina & 96.0 & 30.0 & $\mathrm{LCOCC}$ & Hanuš et al. (2013a) \\
\hline 350 & Ornamenta & 118.3 & 13.5 & STM & Tedesco et al. (2004b) \\
\hline 350 & Ornamenta & 117.2 & 4.5 & NEATM & Usui et al. (2011) \\
\hline 350 & Ornamenta & 109.8 & 12.3 & STM & Ryan \& Woodward (2010) \\
\hline 350 & Ornamenta & 126.3 & 18.4 & NEATM & Ryan \& Woodward (2010) \\
\hline 350 & Ornamenta & 99.5 & 19.1 & NEATM & Masiero et al. (2011) \\
\hline 350 & Ornamenta & 99.5 & 32.0 & NEATM & Masiero et al. (2011) \\
\hline 350 & Ornamenta & 101.6 & 108.0 & NEATM & Nugent et al. (2015) \\
\hline 350 & Ornamenta & 99.4 & 7.3 & OCC & Dunham et al. (2017) \\
\hline 356 & Liguria & 155.0 & 46.5 & STM & Morrison \& Zellner (2007) \\
\hline 356 & Liguria & 126.6 & 31.8 & OCC & Dunham et al. (2017) \\
\hline 356 & Liguria & 131.3 & 7.8 & STM & Tedesco et al. (2004b) \\
\hline 356 & Liguria & 135.7 & 15.2 & STM & Ryan \& Woodward (2010) \\
\hline 356 & Liguria & 135.1 & 21.2 & NEATM & Ryan \& Woodward (2010) \\
\hline 356 & Liguria & 136.6 & 5.6 & STM & Usui et al. (2011) \\
\hline 356 & Liguria & 131.0 & 29.1 & NEATM & Masiero et al. (2011) \\
\hline 356 & Liguria & 145.5 & 4.3 & NEATM & Masiero et al. (2012) \\
\hline 358 & Apollonia & 89.4 & 8.1 & STM & Tedesco et al. (2004b) \\
\hline 358 & Apollonia & 89.4 & 3.7 & NEATM & Usui et al. (2011) \\
\hline 358 & Apollonia & 83.7 & 10.1 & STM & Ryan \& Woodward (2010) \\
\hline 358 & Apollonia & 88.1 & 12.0 & NEATM & Ryan \& Woodward (2010) \\
\hline 358 & Apollonia & 90.5 & 6.4 & NEATM & Masiero et al. (2011) \\
\hline 358 & Apollonia & 93.4 & 77.8 & NEATM & Nugent et al. (2015) \\
\hline 358 & Apollonia & 87.8 & 81.8 & NEATM & Nugent et al. (2015) \\
\hline 362 & Havnia & 85.1 & 3.1 & NEATM & Usui et al. (2011) \\
\hline 362 & Havnia & 89.2 & 6.4 & NEATM & Masiero et al. (2011) \\
\hline 362 & Havnia & 92.0 & 2.6 & NEATM & Masiero et al. (2012) \\
\hline 366 & Vincentina & 93.8 & 9.6 & STM & Tedesco et al. (2004b) \\
\hline 366 & Vincentina & 86.2 & 2.2 & NEATM & Usui et al. (2011) \\
\hline 366 & Vincentina & 93.6 & 15.2 & STM & Ryan \& Woodward (2010) \\
\hline 366 & Vincentina & 98.2 & 13.9 & NEATM & Ryan \& Woodward (2010) \\
\hline 366 & Vincentina & 94.4 & 6.2 & NEATM & Masiero et al. (2011) \\
\hline 366 & Vincentina & 89.5 & 88.4 & NEATM & Nugent et al. (2015) \\
\hline 366 & Vincentina & 85.0 & 39.6 & OCC & Dunham et al. (2017) \\
\hline 366 & Vincentina & 82.0 & 89.7 & OCC & Dunham et al. (2017) \\
\hline 373 & Melusina & 95.8 & 11.1 & STM & Tedesco et al. (2004b) \\
\hline 373 & Melusina & 96.7 & 3.7 & NEATM & Usui et al. (2011) \\
\hline 373 & Melusina & 84.9 & 9.4 & STM & Ryan \& Woodward (2010) \\
\hline 373 & Melusina & 107.7 & 17.4 & NEATM & Ryan \& Woodward (2010) \\
\hline 373 & Melusina & 91.6 & 4.8 & NEATM & Masiero et al. (2011) \\
\hline 373 & Melusina & 98.7 & 2.8 & NEATM & Masiero et al. (2012) \\
\hline 373 & Melusina & 90.4 & 88.8 & NEATM & Nugent et al. (2015) \\
\hline 377 & Campania & 91.1 & 6.0 & STM & Tedesco et al. (2004b) \\
\hline 377 & Campania & 92.6 & 3.3 & NEATM & Usui et al. (2011) \\
\hline 377 & Campania & 75.3 & 7.0 & STM & Ryan \& Woodward (2010) \\
\hline 377 & Campania & 96.4 & 16.9 & NEATM & Ryan \& Woodward (2010) \\
\hline 377 & Campania & 94.0 & 20.1 & NEATM & Masiero et al. (2011) \\
\hline 377 & Campania & 91.0 & 224.7 & OCC & Dunham et al. (2017) \\
\hline 404 & Arsinoe & 101.0 & 30.3 & STM & Morrison \& Zellner (2007) \\
\hline
\end{tabular}


Table D.2. continued.

\begin{tabular}{|c|c|c|c|c|c|}
\hline$\#$ & Name & $\begin{array}{c}\mathcal{D} \\
(\mathrm{km})\end{array}$ & $\begin{array}{c}\delta \mathcal{D} \\
(\mathrm{km})\end{array}$ & Method & Reference \\
\hline 404 & Arsinoe & 97.7 & 4.5 & STM & Tedesco et al. (2004b) \\
\hline 404 & Arsinoe & 98.8 & 9.6 & OCC & Dunham et al. (2017) \\
\hline 404 & Arsinoe & 98.4 & 12.5 & STM & Ryan \& Woodward (2010) \\
\hline 404 & Arsinoe & 102.3 & 13.6 & NEATM & Ryan \& Woodward (2010) \\
\hline 404 & Arsinoe & 93.0 & 3.4 & STM & Usui et al. (2011) \\
\hline 404 & Arsinoe & 98.7 & 10.4 & NEATM & Masiero et al. (2011) \\
\hline 404 & Arsinoe & 108.6 & 3.3 & NEATM & Masiero et al. (2012) \\
\hline 404 & Arsinoe & 101.0 & 15.0 & LCOCC & Hanuš et al. (2013a) \\
\hline 404 & Arsinoe & 85.1 & 84.8 & NEATM & Nugent et al. (2015) \\
\hline 405 & Thia & 124.9 & 6.9 & STM & Tedesco et al. (2004b) \\
\hline 405 & Thia & 129.6 & 11.9 & STM & Ryan \& Woodward (2010) \\
\hline 405 & Thia & 134.9 & 20.2 & NEATM & Ryan \& Woodward (2010) \\
\hline 405 & Thia & 113.3 & 5.2 & STM & Usui et al. (2011) \\
\hline 405 & Thia & 125.0 & 52.3 & NEATM & Masiero et al. (2011) \\
\hline 405 & Thia & 101.5 & 100.6 & NEATM & Nugent et al. (2015) \\
\hline 407 & Arachne & 95.1 & 16.2 & STM & Tedesco et al. (2004b) \\
\hline 407 & Arachne & 97.5 & 4.8 & NEATM & Usui et al. (2011) \\
\hline 407 & Arachne & 82.2 & 8.9 & STM & Ryan \& Woodward (2010) \\
\hline 407 & Arachne & 97.8 & 14.8 & NEATM & Ryan \& Woodward (2010) \\
\hline 407 & Arachne & 86.3 & 62.7 & NEATM & Nugent et al. (2015) \\
\hline 410 & Chloris & 135.0 & 40.5 & STM & Morrison \& Zellner (2007) \\
\hline 410 & Chloris & 123.6 & 16.2 & STM & Tedesco et al. (2004b) \\
\hline 410 & Chloris & 118.0 & 15.7 & STM & Ryan \& Woodward (2010) \\
\hline 410 & Chloris & 124.2 & 16.5 & NEATM & Ryan \& Woodward (2010) \\
\hline 410 & Chloris & 106.7 & 4.3 & STM & Usui et al. (2011) \\
\hline 410 & Chloris & 118.9 & 8.6 & NEATM & Masiero et al. (2011) \\
\hline 410 & Chloris & 96.8 & 87.5 & NEATM & Nugent et al. (2015) \\
\hline 442 & Eichsfeldia & 66.7 & 4.2 & STM & Tedesco et al. (2004b) \\
\hline 442 & Eichsfeldia & 68.7 & 5.9 & STM & Ryan \& Woodward (2010) \\
\hline 442 & Eichsfeldia & 65.9 & 7.7 & NEATM & Ryan \& Woodward (2010) \\
\hline 442 & Eichsfeldia & 65.1 & 2.5 & STM & Usui et al. (2011) \\
\hline 442 & Eichsfeldia & 63.2 & 3.7 & NEATM & Masiero et al. (2011) \\
\hline 442 & Eichsfeldia & 61.6 & 60.5 & NEATM & Nugent et al. (2015) \\
\hline 445 & Edna & 89.3 & 13.6 & STM & Tedesco et al. (2004a) \\
\hline 445 & Edna & 87.2 & 6.3 & STM & Tedesco et al. (2004b) \\
\hline 445 & Edna & 81.4 & 13.0 & STM & Ryan \& Woodward (2010) \\
\hline 445 & Edna & 98.2 & 15.9 & NEATM & Ryan \& Woodward (2010) \\
\hline 445 & Edna & 89.2 & 4.3 & STM & Usui et al. (2011) \\
\hline 445 & Edna & 105.5 & 4.5 & NEATM & Masiero et al. (2011) \\
\hline 445 & Edna & 90.2 & 92.6 & NEATM & Nugent et al. (2015) \\
\hline 445 & Edna & 82.5 & 79.1 & NEATM & Nugent et al. (2015) \\
\hline 481 & Emita & 113.2 & 9.2 & STM & Tedesco et al. (2004b) \\
\hline 481 & Emita & 113.2 & 9.2 & STM & Tedesco et al. (2004a) \\
\hline 481 & Emita & 102.0 & 0.0 & STM & Ryan \& Woodward (2010) \\
\hline 481 & Emita & 108.4 & 0.0 & NEATM & Ryan \& Woodward (2010) \\
\hline 481 & Emita & 103.5 & 5.7 & STM & Usui et al. (2011) \\
\hline 481 & Emita & 121.6 & 117.9 & NEATM & Masiero et al. (2012) \\
\hline 481 & Emita & 104.1 & 119.1 & NEATM & Nugent et al. (2015) \\
\hline 488 & Kreusa & 150.1 & 19.2 & STM & Tedesco et al. (2004b) \\
\hline 488 & Kreusa & 156.0 & 20.8 & STM & Ryan \& Woodward (2010) \\
\hline 488 & Kreusa & 161.6 & 22.2 & NEATM & Ryan \& Woodward (2010) \\
\hline 488 & Kreusa & 172.6 & 7.6 & STM & Usui et al. (2011) \\
\hline 488 & Kreusa & 150.0 & 34.0 & NEATM & Masiero et al. (2011) \\
\hline 488 & Kreusa & 168.1 & 6.2 & NEATM & Masiero et al. (2012) \\
\hline 488 & Kreusa & 143.9 & 156.2 & NEATM & Nugent et al. (2015) \\
\hline 488 & Kreusa & 161.5 & 145.5 & NEATM & Nugent et al. (2015) \\
\hline 490 & Veritas & 108.1 & 13.8 & OCC & Dunham et al. (2017) \\
\hline 490 & Veritas & 115.6 & 16.5 & STM & Tedesco et al. (2004b) \\
\hline
\end{tabular}


B. Carry et al.: Homogeneous internal structure of CM-like asteroid (41) Daphne

Table D.2. continued.

\begin{tabular}{|c|c|c|c|c|c|}
\hline \# & Name & $\begin{array}{c}\mathcal{D} \\
(\mathrm{km})\end{array}$ & $\begin{array}{c}\delta \mathcal{D} \\
(\mathrm{km})\end{array}$ & Method & Reference \\
\hline 490 & Veritas & 131.5 & 12.0 & $\mathrm{IM}$ & Marchis et al. (2006) \\
\hline 490 & Veritas & 102.9 & 15.8 & STM & Ryan \& Woodward (2010) \\
\hline 490 & Veritas & 112.0 & 15.2 & NEATM & Ryan \& Woodward (2010) \\
\hline 490 & Veritas & 112.8 & 5.0 & STM & Usui et al. (2011) \\
\hline 490 & Veritas & 118.8 & 5.5 & NEATM & Masiero et al. (2012) \\
\hline 490 & Veritas & 100.8 & 88.9 & NEATM & Nugent et al. (2015) \\
\hline 490 & Veritas & 108.4 & 108.0 & NEATM & Nugent et al. (2015) \\
\hline 503 & Evelyn & 81.7 & 14.7 & STM & Tedesco et al. (2004b) \\
\hline 503 & Evelyn & 83.4 & 14.7 & STM & Ryan \& Woodward (2010) \\
\hline 503 & Evelyn & 83.6 & 29.7 & NEATM & Ryan \& Woodward (2010) \\
\hline 503 & Evelyn & 90.2 & 3.1 & STM & Usui et al. (2011) \\
\hline 503 & Evelyn & 99.2 & 102.8 & NEATM & Masiero et al. (2012) \\
\hline 521 & Brixia & 115.7 & 6.0 & STM & Tedesco et al. (2004b) \\
\hline 521 & Brixia & 109.3 & 15.6 & OCC & Dunham et al. (2017) \\
\hline 521 & Brixia & 125.4 & 4.9 & NEATM & Usui et al. (2011) \\
\hline 521 & Brixia & 108.2 & 12.6 & STM & Ryan \& Woodward (2010) \\
\hline 521 & Brixia & 120.0 & 15.9 & NEATM & Ryan \& Woodward (2010) \\
\hline 521 & Brixia & 111.9 & 12.2 & NEATM & Masiero et al. (2011) \\
\hline 521 & Brixia & 110.6 & 8.9 & NEATM & Masiero et al. (2011) \\
\hline 521 & Brixia & 104.6 & 91.8 & NEATM & Nugent et al. (2015) \\
\hline 521 & Brixia & 104.0 & 110.9 & NEATM & Nugent et al. (2015) \\
\hline 554 & Peraga & 101.0 & 30.3 & STM & Morrison \& Zellner (2007) \\
\hline 554 & Peraga & 95.9 & 12.3 & STM & Tedesco et al. (2004b) \\
\hline 554 & Peraga & 93.9 & 10.2 & STM & Ryan \& Woodward (2010) \\
\hline 554 & Peraga & 109.1 & 17.0 & NEATM & Ryan \& Woodward (2010) \\
\hline 554 & Peraga & 97.0 & 3.5 & STM & Usui et al. (2011) \\
\hline 554 & Peraga & 102.8 & 81.8 & NEATM & Masiero et al. (2012) \\
\hline 554 & Peraga & 89.4 & 91.0 & NEATM & Nugent et al. (2015) \\
\hline 602 & Marianna & 137.0 & 41.1 & STM & Morrison \& Zellner (2007) \\
\hline 602 & Marianna & 124.7 & 6.6 & STM & Tedesco et al. (2004b) \\
\hline 602 & Marianna & 111.1 & 10.5 & STM & Ryan \& Woodward (2010) \\
\hline 602 & Marianna & 130.1 & 16.6 & NEATM & Ryan \& Woodward (2010) \\
\hline 602 & Marianna & 129.9 & 5.8 & STM & Usui et al. (2011) \\
\hline 602 & Marianna & 126.8 & 6.2 & NEATM & Masiero et al. (2011) \\
\hline 654 & Zelinda & 127.4 & 11.7 & STM & Tedesco et al. (2004b) \\
\hline 654 & Zelinda & 129.1 & 11.4 & STM & Tedesco et al. (2004a) \\
\hline 654 & Zelinda & 119.3 & 52.6 & $\mathrm{OCC}$ & Dunham et al. (2017) \\
\hline 654 & Zelinda & 112.5 & 12.0 & $\mathrm{IM}$ & Marchis et al. (2006) \\
\hline 654 & Zelinda & 138.0 & 13.9 & STM & Ryan \& Woodward (2010) \\
\hline 654 & Zelinda & 134.3 & 18.8 & NEATM & Ryan \& Woodward (2010) \\
\hline 654 & Zelinda & 123.6 & 4.4 & STM & Usui et al. (2011) \\
\hline 654 & Zelinda & 127.0 & 61.4 & NEATM & Masiero et al. (2011) \\
\hline 654 & Zelinda & 160.7 & 4.0 & NEATM & Masiero et al. (2012) \\
\hline 654 & Zelinda & 135.9 & 119.1 & NEATM & Nugent et al. (2015) \\
\hline 654 & Zelinda & 134.8 & 116.8 & NEATM & Nugent et al. (2015) \\
\hline 694 & Ekard & 101.0 & 30.3 & STM & Morrison \& Zellner (2007) \\
\hline 694 & Ekard & 90.8 & 12.0 & STM & Tedesco et al. (2004b) \\
\hline 694 & Ekard & 99.2 & 21.8 & STM & Tedesco et al. (2004a) \\
\hline 694 & Ekard & 104.8 & 8.5 & OCC & Dunham et al. (2017) \\
\hline 694 & Ekard & 95.9 & 110.5 & OCC & Dunham et al. (2017) \\
\hline 694 & Ekard & 90.5 & 13.0 & STM & Ryan \& Woodward (2010) \\
\hline 694 & Ekard & 101.9 & 51.4 & NEATM & Ryan \& Woodward (2010) \\
\hline 694 & Ekard & 63.9 & 10.9 & STM & Ryan \& Woodward (2010) \\
\hline 694 & Ekard & 92.8 & 17.0 & NEATM & Ryan \& Woodward (2010) \\
\hline 694 & Ekard & 92.1 & 3.8 & STM & Usui et al. (2011) \\
\hline 694 & Ekard & 121.9 & 2.2 & STM & Masiero et al. (2012) \\
\hline 694 & Ekard & 98.9 & 76.2 & NEATM & Nugent et al. (2015) \\
\hline 735 & Marghanna & 74.3 & 4.8 & STM & Tedesco et al. (2004b) \\
\hline
\end{tabular}


Table D.2. continued.

\begin{tabular}{|c|c|c|c|c|c|}
\hline$\#$ & Name & $\begin{array}{c}\mathcal{D} \\
(\mathrm{km})\end{array}$ & $\begin{array}{c}\delta \mathcal{D} \\
(\mathrm{km})\end{array}$ & Method & Reference \\
\hline 735 & Marghanna & 65.1 & 5.9 & STM & Ryan \& Woodward (2010) \\
\hline 735 & Marghanna & 76.9 & 11.2 & NEATM & Ryan \& Woodward (2010) \\
\hline 735 & Marghanna & 78.7 & 4.9 & STM & Usui et al. (2011) \\
\hline 735 & Marghanna & 70.6 & 3.7 & NEATM & Masiero et al. (2011) \\
\hline 735 & Marghanna & 70.8 & 3.8 & NEATM & Masiero et al. (2011) \\
\hline 735 & Marghanna & 57.2 & 78.2 & NEATM & Nugent et al. (2015) \\
\hline 751 & Faina & 110.5 & 12.9 & STM & Tedesco et al. (2004b) \\
\hline 751 & Faina & 109.6 & 23.7 & STM & Ryan \& Woodward (2010) \\
\hline 751 & Faina & 122.5 & 18.7 & NEATM & Ryan \& Woodward (2010) \\
\hline 751 & Faina & 106.8 & 3.8 & STM & Usui et al. (2011) \\
\hline 751 & Faina & 106.3 & 4.9 & NEATM & Masiero et al. (2011) \\
\hline 751 & Faina & 123.7 & 130.4 & NEATM & Nugent et al. (2015) \\
\hline 776 & Berbericia & 151.2 & 12.0 & STM & Tedesco et al. (2004b) \\
\hline 776 & Berbericia & 172.4 & 80.4 & OCC & Dunham et al. (2017) \\
\hline 776 & Berbericia & 155.8 & 15.4 & STM & Ryan \& Woodward (2010) \\
\hline 776 & Berbericia & 165.9 & 31.1 & NEATM & Ryan \& Woodward (2010) \\
\hline 776 & Berbericia & 149.8 & 5.3 & STM & Usui et al. (2011) \\
\hline 776 & Berbericia & 151.1 & 12.3 & NEATM & Masiero et al. (2011) \\
\hline 776 & Berbericia & 135.1 & 102.9 & NEATM & Nugent et al. (2015) \\
\hline 788 & Hohensteina & 103.7 & 10.2 & STM & Tedesco et al. (2004b) \\
\hline 788 & Hohensteina & 105.5 & 13.5 & OCC & Dunham et al. (2017) \\
\hline 788 & Hohensteina & 100.6 & 7.5 & OCC & Dunham et al. (2017) \\
\hline 788 & Hohensteina & 118.3 & 5.0 & NEATM & Usui et al. (2011) \\
\hline 788 & Hohensteina & 108.1 & 11.2 & STM & Ryan \& Woodward (2010) \\
\hline 788 & Hohensteina & 121.0 & 23.3 & NEATM & Ryan \& Woodward (2010) \\
\hline 788 & Hohensteina & 118.3 & 8.2 & NEATM & Masiero et al. (2011) \\
\hline 788 & Hohensteina & 125.8 & 4.5 & NEATM & Masiero et al. (2012) \\
\hline 788 & Hohensteina & 101.2 & 97.4 & NEATM & Nugent et al. (2015) \\
\hline 791 & Ani & 103.5 & 5.7 & STM & Tedesco et al. (2004b) \\
\hline 791 & Ani & 82.5 & 9.6 & OCC & Dunham et al. (2017) \\
\hline 791 & Ani & 85.9 & 26.9 & OCC & Dunham et al. (2017) \\
\hline 791 & Ani & 97.9 & 3.4 & NEATM & Usui et al. (2011) \\
\hline 791 & Ani & 93.4 & 8.5 & STM & Ryan \& Woodward (2010) \\
\hline 791 & Ani & 115.0 & 14.0 & NEATM & Ryan \& Woodward (2010) \\
\hline 791 & Ani & 82.5 & 17.9 & NEATM & Masiero et al. (2011) \\
\hline 791 & Ani & 116.9 & 3.1 & NEATM & Masiero et al. (2012) \\
\hline 791 & Ani & 83.3 & 63.9 & NEATM & Nugent et al. (2015) \\
\hline 914 & Palisana & 80.5 & 5.8 & STM & Tedesco et al. (2004a) \\
\hline 914 & Palisana & 76.6 & 5.1 & STM & Tedesco et al. (2004b) \\
\hline 914 & Palisana & 91.2 & 7.8 & OCC & Dunham et al. (2017) \\
\hline 914 & Palisana & 76.5 & 15.0 & IM & Marchis et al. (2006) \\
\hline 914 & Palisana & 67.0 & 6.3 & STM & Ryan \& Woodward (2010) \\
\hline 914 & Palisana & 83.6 & 11.3 & NEATM & Ryan \& Woodward (2010) \\
\hline 914 & Palisana & 97.3 & 4.5 & STM & Usui et al. (2011) \\
\hline 914 & Palisana & 77.0 & 39.4 & NEATM & Masiero et al. (2011) \\
\hline 914 & Palisana & 78.9 & 61.3 & NEATM & Nugent et al. (2015) \\
\hline 1467 & Mashona & 95.1 & 3.9 & NEATM & Usui et al. (2011) \\
\hline
\end{tabular}


Table D.3. The mass $(\mathcal{M})$ for all the $\mathrm{Ch} / \mathrm{Cgh}$ asteroids available in the literature.

\begin{tabular}{|c|c|c|c|c|c|}
\hline \# & Name & $\begin{array}{c}\mathcal{M} \\
(\mathrm{kg})\end{array}$ & $\begin{array}{l}\delta \mathcal{M} \\
(\mathrm{kg})\end{array}$ & Method & Reference \\
\hline 13 & Egeria & $1.63 \times 10^{19}$ & $9.54 \times 10^{18}$ & DEFL & Baer et al. (2008) \\
\hline 13 & Egeria & $6.17 \times 10^{18}$ & $1.85 \times 10^{18}$ & EPHEM & Folkner et al. (2009) \\
\hline 13 & Egeria & $1.59 \times 10^{19}$ & $1.31 \times 10^{19}$ & DEFL & Baer et al. (2011) \\
\hline 13 & Egeria & $1.29 \times 10^{19}$ & $1.41 \times 10^{19}$ & DEFL & Zielenbach (2011) \\
\hline 13 & Egeria & $7.39 \times 10^{18}$ & $9.72 \times 10^{18}$ & DEFL & Zielenbach (2011) \\
\hline 13 & Egeria & $6.07 \times 10^{18}$ & $9.60 \times 10^{18}$ & DEFL & Zielenbach (2011) \\
\hline 13 & Egeria & $8.26 \times 10^{18}$ & $1.83 \times 10^{19}$ & DEFL & Zielenbach (2011) \\
\hline 13 & Egeria & $9.37 \times 10^{18}$ & $4.65 \times 10^{18}$ & EPHEM & Fienga et al. (2013) \\
\hline 13 & Egeria & $1.23 \times 10^{19}$ & $9.90 \times 10^{18}$ & EPHEM & Kuchynka \& Folkner (2013) \\
\hline 13 & Egeria & $9.37 \times 10^{18}$ & $7.08 \times 10^{18}$ & EPHEM & Fienga et al. (2014) \\
\hline 13 & Egeria & $9.35 \times 10^{18}$ & $2.39 \times 10^{18}$ & DEFL & Goffin (2014) \\
\hline 13 & Egeria & $9.62 \times 10^{18}$ & $4.47 \times 10^{18}$ & DEFL & Kochetova \& Chernetenko (2014) \\
\hline 13 & Egeria & $1.04 \times 10^{19}$ & $4.65 \times 10^{18}$ & EPHEM & Viswanathan et al. (2017) \\
\hline 13 & Egeria & $2.25 \times 10^{19}$ & $7.83 \times 10^{19}$ & DEFL & Siltala \& Granvik (2017) \\
\hline 13 & Egeria & $1.08 \times 10^{19}$ & $4.38 \times 10^{18}$ & EPHEM & Fienga (2018, priv. comm.) \\
\hline 19 & Fortuna & $1.27 \times 10^{19}$ & $1.49 \times 10^{18}$ & DEFL & Baer et al. (2008) \\
\hline 19 & Fortuna & $4.02 \times 10^{18}$ & $1.19 \times 10^{18}$ & EPHEM & Fienga et al. (2009) \\
\hline 19 & Fortuna & $6.94 \times 10^{18}$ & $2.08 \times 10^{18}$ & EPHEM & Folkner et al. (2009) \\
\hline 19 & Fortuna & $6.37 \times 10^{18}$ & $8.70 \times 10^{18}$ & DEFL & Somenzi et al. (2010) \\
\hline 19 & Fortuna & $8.31 \times 10^{18}$ & $2.15 \times 10^{18}$ & DEFL & Baer et al. (2011) \\
\hline 19 & Fortuna & $6.37 \times 10^{18}$ & $3.15 \times 10^{18}$ & EPHEM & Konopliv et al. (2011) \\
\hline 19 & Fortuna & $1.00 \times 10^{19}$ & $3.24 \times 10^{18}$ & DEFL & Zielenbach (2011) \\
\hline 19 & Fortuna & $1.02 \times 10^{19}$ & $2.84 \times 10^{18}$ & DEFL & Zielenbach (2011) \\
\hline 19 & Fortuna & $1.01 \times 10^{19}$ & $2.81 \times 10^{18}$ & DEFL & Zielenbach (2011) \\
\hline 19 & Fortuna & $1.05 \times 10^{19}$ & $3.69 \times 10^{18}$ & DEFL & Zielenbach (2011) \\
\hline 19 & Fortuna & $8.35 \times 10^{18}$ & $1.79 \times 10^{18}$ & EPHEM & Fienga et al. (2011) \\
\hline 19 & Fortuna & $9.73 \times 10^{18}$ & $3.03 \times 10^{18}$ & EPHEM & Fienga et al. (2013) \\
\hline 19 & Fortuna & $7.79 \times 10^{18}$ & $2.70 \times 10^{18}$ & EPHEM & Kuchynka \& Folkner (2013) \\
\hline 19 & Fortuna & $8.67 \times 10^{18}$ & $7.77 \times 10^{17}$ & EPHEM & Pitjeva (2013) \\
\hline 19 & Fortuna & $8.00 \times 10^{18}$ & $2.81 \times 10^{18}$ & EPHEM & Fienga et al. (2014) \\
\hline 19 & Fortuna & $8.95 \times 10^{18}$ & $5.97 \times 10^{17}$ & DEFL & Goffin (2014) \\
\hline 19 & Fortuna & $8.83 \times 10^{18}$ & $1.25 \times 10^{18}$ & DEFL & Kochetova \& Chernetenko (2014) \\
\hline 19 & Fortuna & $1.03 \times 10^{19}$ & $1.68 \times 10^{18}$ & EPHEM & Viswanathan et al. (2017) \\
\hline 19 & Fortuna & $2.80 \times 10^{18}$ & $9.33 \times 10^{18}$ & DEFL & Siltala \& Granvik (2017) \\
\hline 19 & Fortuna & $2.21 \times 10^{19}$ & $3.03 \times 10^{19}$ & DEFL & Siltala \& Granvik (2017) \\
\hline 19 & Fortuna & $1.10 \times 10^{19}$ & $1.90 \times 10^{18}$ & EPHEM & Baer \& Chesley (2017) \\
\hline 19 & Fortuna & $1.17 \times 10^{19}$ & $1.42 \times 10^{18}$ & EPHEM & Fienga (2018, priv. comm.) \\
\hline 34 & Circe & $3.68 \times 10^{18}$ & $4.53 \times 10^{18}$ & EPHEM & Fienga et al. (2011) \\
\hline 34 & Circe & $2.89 \times 10^{18}$ & $3.63 \times 10^{18}$ & EPHEM & Fienga et al. (2013) \\
\hline 34 & Circe & $4.18 \times 10^{18}$ & $1.19 \times 10^{18}$ & DEFL & Goffin (2014) \\
\hline 34 & Circe & $5.01 \times 10^{18}$ & $4.86 \times 10^{18}$ & EPHEM & Viswanathan et al. (2017) \\
\hline 34 & Circe & $5.96 \times 10^{18}$ & $5.19 \times 10^{18}$ & EPHEM & Fienga (2018, priv. comm.) \\
\hline 38 & Leda & $3.18 \times 10^{18}$ & $1.79 \times 10^{18}$ & DEFL & Goffin (2014) \\
\hline 38 & Leda & $6.03 \times 10^{18}$ & $4.38 \times 10^{18}$ & EPHEM & Viswanathan et al. (2017) \\
\hline 38 & Leda & $7.07 \times 10^{18}$ & $4.44 \times 10^{18}$ & EPHEM & Fienga (2018, priv. comm.) \\
\hline 41 & Daphne & $1.05 \times 10^{19}$ & $2.99 \times 10^{18}$ & EPHEM & Fienga et al. (2009) \\
\hline 41 & Daphne & $7.90 \times 10^{18}$ & $2.37 \times 10^{18}$ & EPHEM & Folkner et al. (2009) \\
\hline 41 & Daphne & $8.43 \times 10^{18}$ & $1.06 \times 10^{19}$ & EPHEM & Konopliv et al. (2011) \\
\hline 41 & Daphne & $1.82 \times 10^{19}$ & $2.16 \times 10^{19}$ & DEFL & Zielenbach (2011) \\
\hline 41 & Daphne & $3.02 \times 10^{17}$ & $1.70 \times 10^{19}$ & DEFL & Zielenbach (2011) \\
\hline 41 & Daphne & $4.76 \times 10^{18}$ & $1.65 \times 10^{19}$ & DEFL & Zielenbach (2011) \\
\hline 41 & Daphne & $1.21 \times 10^{19}$ & $3.15 \times 10^{19}$ & DEFL & Zielenbach (2011) \\
\hline 41 & Daphne & $1.02 \times 10^{19}$ & $3.57 \times 10^{18}$ & EPHEM & Fienga et al. (2011) \\
\hline
\end{tabular}

Notes. For each, the $3 \sigma$ uncertainty, method, and bibliographic reference are reported. The methods are DEFL: Deflection, EPHEM: Ephemeris, BIMG: Binary: Imaging, and BGENO: Binary: Genoid. 
Table D.3. continued.

\begin{tabular}{|c|c|c|c|c|c|}
\hline$\#$ & Name & $\underset{(\mathrm{kg})}{\mathcal{M}}$ & $\begin{array}{l}\delta \mathcal{M} \\
(\mathrm{kg})\end{array}$ & Method & Reference \\
\hline 41 & Daphne & $7.79 \times 10^{18}$ & $5.40 \times 10^{18}$ & EPHEM & Kuchynka \& Folkner (2013) \\
\hline 41 & Daphne & $8.29 \times 10^{18}$ & $2.62 \times 10^{18}$ & EPHEM & Pitjeva (2013) \\
\hline 41 & Daphne & $7.13 \times 10^{18}$ & $2.01 \times 10^{18}$ & EPHEM & Fienga et al. (2014) \\
\hline 41 & Daphne & $9.35 \times 10^{18}$ & $4.17 \times 10^{18}$ & DEFL & Goffin (2014) \\
\hline 41 & Daphne & $9.78 \times 10^{18}$ & $1.38 \times 10^{19}$ & DEFL & Kochetova \& Chernetenko (2014) \\
\hline 41 & Daphne & $4.44 \times 10^{18}$ & $2.52 \times 10^{18}$ & EPHEM & Fienga (2018, priv. comm.) \\
\hline 41 & Daphne & $5.95 \times 10^{18}$ & $1.70 \times 10^{18}$ & BGENO & This work \\
\hline 48 & Doris & $1.21 \times 10^{19}$ & $1.79 \times 10^{19}$ & DEFL & Kochetova (2004) \\
\hline 48 & Doris & $5.89 \times 10^{18}$ & $1.45 \times 10^{19}$ & DEFL & Zielenbach (2011) \\
\hline 48 & Doris & $3.78 \times 10^{18}$ & $1.12 \times 10^{19}$ & DEFL & Zielenbach (2011) \\
\hline 48 & Doris & $3.84 \times 10^{18}$ & $1.11 \times 10^{19}$ & DEFL & Zielenbach (2011) \\
\hline 48 & Doris & $7.94 \times 10^{18}$ & $1.92 \times 10^{19}$ & DEFL & Zielenbach (2011) \\
\hline 48 & Doris & $2.40 \times 10^{19}$ & $2.05 \times 10^{19}$ & EPHEM & Fienga et al. (2011) \\
\hline 48 & Doris & $5.99 \times 10^{15}$ & $1.13 \times 10^{17}$ & EPHEM & Fienga et al. (2014) \\
\hline 48 & Doris & $7.76 \times 10^{18}$ & $1.79 \times 10^{18}$ & DEFL & Goffin (2014) \\
\hline 48 & Doris & $1.75 \times 10^{19}$ & $1.54 \times 10^{19}$ & EPHEM & Fienga (2018, priv. comm.) \\
\hline 49 & Pales & $2.69 \times 10^{18}$ & $1.49 \times 10^{18}$ & DEFL & Baer et al. (2008) \\
\hline 49 & Pales & $5.07 \times 10^{18}$ & $1.16 \times 10^{19}$ & DEFL & Zielenbach (2011) \\
\hline 49 & Pales & $8.11 \times 10^{18}$ & $8.04 \times 10^{18}$ & DEFL & Zielenbach (2011) \\
\hline 49 & Pales & $7.61 \times 10^{18}$ & $8.01 \times 10^{18}$ & DEFL & Zielenbach (2011) \\
\hline 49 & Pales & $4.93 \times 10^{18}$ & $1.47 \times 10^{19}$ & DEFL & Zielenbach (2011) \\
\hline 49 & Pales & $5.37 \times 10^{18}$ & $1.79 \times 10^{18}$ & DEFL & Goffin (2014) \\
\hline 49 & Pales & $7.59 \times 10^{18}$ & $5.52 \times 10^{18}$ & EPHEM & Fienga (2018, priv. comm.) \\
\hline 50 & Virginia & $1.95 \times 10^{18}$ & $2.18 \times 10^{18}$ & EPHEM & Fienga et al. (2011) \\
\hline 50 & Virginia & $5.97 \times 10^{17}$ & $1.79 \times 10^{17}$ & DEFL & Goffin (2014) \\
\hline 50 & Virginia & $1.46 \times 10^{18}$ & $1.16 \times 10^{18}$ & EPHEM & Fienga (2018, priv. comm.) \\
\hline 51 & Nemausa & $2.16 \times 10^{18}$ & $6.48 \times 10^{17}$ & EPHEM & Folkner et al. (2009) \\
\hline 51 & Nemausa & $4.55 \times 10^{18}$ & $8.16 \times 10^{18}$ & DEFL & Zielenbach (2011) \\
\hline 51 & Nemausa & $3.39 \times 10^{18}$ & $4.86 \times 10^{18}$ & DEFL & Zielenbach (2011) \\
\hline 51 & Nemausa & $3.36 \times 10^{18}$ & $4.86 \times 10^{18}$ & DEFL & Zielenbach (2011) \\
\hline 51 & Nemausa & $4.25 \times 10^{18}$ & $6.24 \times 10^{18}$ & DEFL & Zielenbach (2011) \\
\hline 51 & Nemausa & $5.63 \times 10^{18}$ & $3.90 \times 10^{18}$ & EPHEM & Fienga et al. (2011) \\
\hline 51 & Nemausa & $1.79 \times 10^{16}$ & $2.69 \times 10^{16}$ & EPHEM & Fienga et al. (2013) \\
\hline 51 & Nemausa & $4.76 \times 10^{15}$ & $1.65 \times 10^{18}$ & EPHEM & Fienga et al. (2014) \\
\hline 51 & Nemausa & $2.78 \times 10^{18}$ & $1.19 \times 10^{18}$ & DEFL & Goffin (2014) \\
\hline 51 & Nemausa & $4.79 \times 10^{18}$ & $2.53 \times 10^{18}$ & EPHEM & Baer \& Chesley (2017) \\
\hline 51 & Nemausa & $4.22 \times 10^{18}$ & $2.26 \times 10^{18}$ & EPHEM & Baer \& Chesley (2017) \\
\hline 51 & Nemausa & $3.98 \times 10^{18}$ & $2.54 \times 10^{18}$ & EPHEM & Fienga (2018, priv. comm.) \\
\hline 54 & Alexandra & $1.08 \times 10^{19}$ & $1.83 \times 10^{19}$ & DEFL & Zielenbach (2011) \\
\hline 54 & Alexandra & $1.74 \times 10^{18}$ & $9.57 \times 10^{18}$ & DEFL & Zielenbach (2011) \\
\hline 54 & Alexandra & $2.94 \times 10^{18}$ & $9.45 \times 10^{18}$ & DEFL & Zielenbach (2011) \\
\hline 54 & Alexandra & $6.44 \times 10^{18}$ & $2.08 \times 10^{19}$ & DEFL & Zielenbach (2011) \\
\hline 54 & Alexandra & $1.03 \times 10^{19}$ & $5.22 \times 10^{18}$ & EPHEM & Fienga et al. (2011) \\
\hline 54 & Alexandra & $1.67 \times 10^{19}$ & $6.45 \times 10^{18}$ & EPHEM & Fienga et al. (2013) \\
\hline 54 & Alexandra & $1.21 \times 10^{19}$ & $3.42 \times 10^{18}$ & EPHEM & Viswanathan et al. (2017) \\
\hline 54 & Alexandra & $1.62 \times 10^{18}$ & $2.02 \times 10^{18}$ & EPHEM & Fienga (2018, priv. comm.) \\
\hline 58 & Concordia & $1.28 \times 10^{17}$ & $1.92 \times 10^{17}$ & EPHEM & Fienga (2018, priv. comm.) \\
\hline 62 & Erato & $1.38 \times 10^{17}$ & $2.07 \times 10^{17}$ & EPHEM & Fienga (2018, priv. comm.) \\
\hline 70 & Panopaea & $4.33 \times 10^{18}$ & $3.27 \times 10^{18}$ & EPHEM & Fienga et al. (2011) \\
\hline 70 & Panopaea & $3.38 \times 10^{18}$ & $1.19 \times 10^{18}$ & DEFL & Goffin (2014) \\
\hline 70 & Panopaea & $4.67 \times 10^{18}$ & $2.95 \times 10^{18}$ & EPHEM & Fienga (2018, priv. comm.) \\
\hline 78 & Diana & $1.27 \times 10^{18}$ & $3.81 \times 10^{17}$ & EPHEM & Folkner et al. (2009) \\
\hline 78 & Diana & $5.09 \times 10^{18}$ & $3.39 \times 10^{18}$ & EPHEM & Fienga et al. (2013) \\
\hline 78 & Diana & $4.29 \times 10^{17}$ & $8.07 \times 10^{17}$ & EPHEM & Viswanathan et al. (2017) \\
\hline 78 & Diana & $3.76 \times 10^{17}$ & $5.34 \times 10^{17}$ & EPHEM & Fienga (2018, priv. comm.) \\
\hline
\end{tabular}


Table D.3. continued.

\begin{tabular}{|c|c|c|c|c|c|}
\hline$\#$ & Name & $\begin{array}{c}\mathcal{M} \\
(\mathrm{kg})\end{array}$ & $\begin{array}{l}\delta \mathcal{M} \\
(\mathrm{kg})\end{array}$ & Method & Reference \\
\hline 84 & Klio & $5.45 \times 10^{17}$ & $8.04 \times 10^{17}$ & EPHEM & Viswanathan et al. (2017) \\
\hline 84 & Klio & $7.60 \times 10^{17}$ & $7.32 \times 10^{17}$ & EPHEM & Fienga (2018, priv. comm.) \\
\hline 91 & Aegina & $6.07 \times 10^{17}$ & $8.94 \times 10^{17}$ & EPHEM & Viswanathan et al. (2017) \\
\hline 91 & Aegina & $2.00 \times 10^{17}$ & $2.99 \times 10^{17}$ & EPHEM & Fienga (2018, priv. comm.) \\
\hline 95 & Arethusa & $4.18 \times 10^{18}$ & $2.39 \times 10^{18}$ & DEFL & Goffin (2014) \\
\hline 95 & Arethusa & $6.86 \times 10^{18}$ & $8.91 \times 10^{18}$ & EPHEM & Viswanathan et al. (2017) \\
\hline 95 & Arethusa & $7.46 \times 10^{18}$ & $9.30 \times 10^{18}$ & EPHEM & Fienga (2018, priv. comm.) \\
\hline 98 & Ianthe & $8.24 \times 10^{17}$ & $2.47 \times 10^{17}$ & EPHEM & Folkner et al. (2009) \\
\hline 98 & Ianthe & $1.47 \times 10^{18}$ & $2.07 \times 10^{18}$ & EPHEM & Fienga et al. (2011) \\
\hline 98 & Ianthe & $1.65 \times 10^{18}$ & $2.23 \times 10^{18}$ & EPHEM & Viswanathan et al. (2017) \\
\hline 98 & Ianthe & $1.55 \times 10^{18}$ & $2.02 \times 10^{18}$ & EPHEM & Fienga (2018, priv. comm.) \\
\hline 104 & Klymene & $1.79 \times 10^{18}$ & $1.19 \times 10^{18}$ & DEFL & Goffin (2014) \\
\hline 104 & Klymene & $3.78 \times 10^{18}$ & $4.80 \times 10^{18}$ & EPHEM & Fienga (2018, priv. comm.) \\
\hline 105 & Artemis & $1.32 \times 10^{18}$ & $3.96 \times 10^{17}$ & EPHEM & Folkner et al. (2009) \\
\hline 105 & Artemis & $2.89 \times 10^{18}$ & $2.53 \times 10^{18}$ & EPHEM & Fienga et al. (2011) \\
\hline 105 & Artemis & $6.06 \times 10^{18}$ & $3.81 \times 10^{18}$ & EPHEM & Fienga et al. (2013) \\
\hline 105 & Artemis & $2.63 \times 10^{18}$ & $1.82 \times 10^{18}$ & EPHEM & Viswanathan et al. (2017) \\
\hline 105 & Artemis & $1.91 \times 10^{18}$ & $1.82 \times 10^{18}$ & EPHEM & Fienga (2018, priv. comm.) \\
\hline 106 & Dione & $3.04 \times 10^{17}$ & $1.08 \times 10^{19}$ & DEFL & Zielenbach (2011) \\
\hline 106 & Dione & $4.29 \times 10^{18}$ & $7.92 \times 10^{18}$ & DEFL & Zielenbach (2011) \\
\hline 106 & Dione & $3.52 \times 10^{18}$ & $7.86 \times 10^{18}$ & DEFL & Zielenbach (2011) \\
\hline 106 & Dione & $3.77 \times 10^{18}$ & $1.74 \times 10^{19}$ & DEFL & Zielenbach (2011) \\
\hline 106 & Dione & $7.70 \times 10^{18}$ & $2.44 \times 10^{18}$ & EPHEM & Fienga et al. (2013) \\
\hline 106 & Dione & $3.58 \times 10^{18}$ & $1.79 \times 10^{18}$ & DEFL & Goffin (2014) \\
\hline 106 & Dione & $1.08 \times 10^{17}$ & $1.62 \times 10^{17}$ & EPHEM & Fienga (2018, priv. comm.) \\
\hline 109 & Felicitas & $3.20 \times 10^{17}$ & $1.49 \times 10^{18}$ & EPHEM & Fienga et al. (2013) \\
\hline 109 & Felicitas & $2.21 \times 10^{18}$ & $1.55 \times 10^{18}$ & EPHEM & Fienga et al. (2014) \\
\hline 109 & Felicitas & $1.65 \times 10^{17}$ & $2.44 \times 10^{17}$ & EPHEM & Fienga (2018, priv. comm.) \\
\hline 111 & Ate & $1.99 \times 10^{18}$ & $5.97 \times 10^{17}$ & DEFL & Krasinsky et al. (2001) \\
\hline 111 & Ate & $1.67 \times 10^{20}$ & $1.13 \times 10^{20}$ & DEFL & Ivantsov (2008) \\
\hline 111 & Ate & $1.74 \times 10^{18}$ & $5.22 \times 10^{17}$ & EPHEM & Folkner et al. (2009) \\
\hline 111 & Ate & $2.71 \times 10^{18}$ & $7.47 \times 10^{18}$ & DEFL & Zielenbach (2011) \\
\hline 111 & Ate & $3.42 \times 10^{17}$ & $4.95 \times 10^{18}$ & DEFL & Zielenbach (2011) \\
\hline 111 & Ate & $8.16 \times 10^{17}$ & $4.98 \times 10^{18}$ & DEFL & Zielenbach (2011) \\
\hline 111 & Ate & $8.93 \times 10^{18}$ & $7.05 \times 10^{18}$ & EPHEM & Fienga et al. (2013) \\
\hline 111 & Ate & $5.87 \times 10^{17}$ & $5.94 \times 10^{18}$ & EPHEM & Fienga et al. (2014) \\
\hline 111 & Ate & $3.05 \times 10^{18}$ & $3.45 \times 10^{18}$ & EPHEM & Viswanathan et al. (2017) \\
\hline 111 & Ate & $3.60 \times 10^{18}$ & $3.15 \times 10^{18}$ & EPHEM & Fienga (2018, priv. comm.) \\
\hline 112 & Iphigenia & $4.52 \times 10^{17}$ & $6.36 \times 10^{17}$ & EPHEM & Fienga (2018, priv. comm.) \\
\hline 121 & Hermione & $9.35 \times 10^{18}$ & $4.77 \times 10^{18}$ & DEFL & Viateau $(2000)$ \\
\hline 121 & Hermione & $5.38 \times 10^{18}$ & $8.94 \times 10^{17}$ & BIMG & Marchis et al. (2005) \\
\hline 121 & Hermione & $4.70 \times 10^{18}$ & $6.00 \times 10^{17}$ & BIMG & Descamps et al. (2009) \\
\hline 121 & Hermione & $5.12 \times 10^{18}$ & $6.66 \times 10^{18}$ & DEFL & Zielenbach (2011) \\
\hline 121 & Hermione & $6.01 \times 10^{18}$ & $5.10 \times 10^{18}$ & DEFL & Zielenbach (2011) \\
\hline 121 & Hermione & $4.58 \times 10^{18}$ & $6.39 \times 10^{18}$ & DEFL & Zielenbach (2011) \\
\hline 121 & Hermione & $6.27 \times 10^{18}$ & $6.84 \times 10^{18}$ & DEFL & Zielenbach (2011) \\
\hline 121 & Hermione & $3.18 \times 10^{18}$ & $1.19 \times 10^{18}$ & DEFL & Goffin (2014) \\
\hline 121 & Hermione & $4.77 \times 10^{18}$ & $2.39 \times 10^{18}$ & DEFL & Kretlow (2014) \\
\hline 121 & Hermione & $6.69 \times 10^{18}$ & $7.65 \times 10^{18}$ & EPHEM & Viswanathan et al. (2017) \\
\hline 121 & Hermione & $5.53 \times 10^{18}$ & $3.13 \times 10^{18}$ & EPHEM & Baer \& Chesley (2017) \\
\hline 121 & Hermione & $7.29 \times 10^{18}$ & $7.44 \times 10^{18}$ & EPHEM & Fienga (2018, priv. comm.) \\
\hline 127 & Johanna & $3.08 \times 10^{18}$ & $4.05 \times 10^{18}$ & EPHEM & Fienga et al. (2011) \\
\hline 127 & Johanna & $1.67 \times 10^{18}$ & $4.14 \times 10^{18}$ & EPHEM & Fienga (2018, priv. comm.) \\
\hline 130 & Elektra & $6.60 \times 10^{18}$ & $1.19 \times 10^{18}$ & BIMG & Marchis et al. (2008b) \\
\hline 130 & Elektra & $1.61 \times 10^{19}$ & $2.51 \times 10^{19}$ & DEFL & Zielenbach (2011) \\
\hline
\end{tabular}


Table D.3. continued.

\begin{tabular}{|c|c|c|c|c|c|}
\hline$\#$ & Name & $\begin{array}{c}\mathcal{M} \\
(\mathrm{kg})\end{array}$ & $\begin{array}{l}\delta \mathcal{M} \\
(\mathrm{kg})\end{array}$ & Method & Reference \\
\hline 130 & Elektra & $1.00 \times 10^{19}$ & $1.97 \times 10^{19}$ & DEFL & Zielenbach (2011) \\
\hline 130 & Elektra & $6.93 \times 10^{18}$ & $1.93 \times 10^{19}$ & DEFL & Zielenbach (2011) \\
\hline 130 & Elektra & $1.34 \times 10^{19}$ & $3.90 \times 10^{19}$ & DEFL & Zielenbach (2011) \\
\hline 130 & Elektra & $2.19 \times 10^{17}$ & $3.57 \times 10^{17}$ & EPHEM & Fienga et al. (2011) \\
\hline 130 & Elektra & $1.39 \times 10^{19}$ & $4.77 \times 10^{18}$ & DEFL & Goffin (2014) \\
\hline 130 & Elektra & $9.41 \times 10^{18}$ & $6.27 \times 10^{18}$ & EPHEM & Viswanathan et al. (2017) \\
\hline 130 & Elektra & $7.46 \times 10^{18}$ & $6.27 \times 10^{18}$ & EPHEM & Fienga (2018, priv. comm.) \\
\hline 130 & Elektra & $6.20 \times 10^{18}$ & $6.00 \times 10^{16}$ & BIMG & Yang et al. (2016) \\
\hline 134 & Sophrosyne & $2.02 \times 10^{18}$ & $2.21 \times 10^{18}$ & EPHEM & Fienga et al. (2013) \\
\hline 134 & Sophrosyne & $4.92 \times 10^{18}$ & $3.45 \times 10^{18}$ & EPHEM & Viswanathan et al. (2017) \\
\hline 134 & Sophrosyne & $5.68 \times 10^{17}$ & $8.34 \times 10^{17}$ & EPHEM & Fienga (2018, priv. comm.) \\
\hline 141 & Lumen & $8.31 \times 10^{18}$ & $2.80 \times 10^{18}$ & EPHEM & Viswanathan et al. (2017) \\
\hline 141 & Lumen & $3.27 \times 10^{18}$ & $2.80 \times 10^{18}$ & EPHEM & Fienga (2018, priv. comm.) \\
\hline 144 & Vibilia & $4.63 \times 10^{18}$ & $8.52 \times 10^{18}$ & DEFL & Zielenbach (2011) \\
\hline 144 & Vibilia & $5.32 \times 10^{18}$ & $5.58 \times 10^{18}$ & DEFL & Zielenbach (2011) \\
\hline 144 & Vibilia & $4.95 \times 10^{18}$ & $5.55 \times 10^{18}$ & DEFL & Zielenbach (2011) \\
\hline 144 & Vibilia & $4.62 \times 10^{18}$ & $9.18 \times 10^{18}$ & DEFL & Zielenbach (2011) \\
\hline 144 & Vibilia & $1.61 \times 10^{15}$ & $5.97 \times 10^{15}$ & EPHEM & Fienga et al. (2014) \\
\hline 144 & Vibilia & $3.38 \times 10^{18}$ & $1.19 \times 10^{18}$ & DEFL & Goffin (2014) \\
\hline 144 & Vibilia & $4.69 \times 10^{18}$ & $2.49 \times 10^{18}$ & EPHEM & Fienga (2018, priv. comm.) \\
\hline 145 & Adeona & $2.26 \times 10^{18}$ & $6.78 \times 10^{17}$ & EPHEM & Folkner et al. (2009) \\
\hline 145 & Adeona & $1.73 \times 10^{17}$ & $7.17 \times 10^{18}$ & DEFL & Zielenbach (2011) \\
\hline 145 & Adeona & $2.10 \times 10^{18}$ & $8.91 \times 10^{18}$ & DEFL & Zielenbach (2011) \\
\hline 145 & Adeona & $2.04 \times 10^{15}$ & $6.57 \times 10^{15}$ & EPHEM & Fienga et al. (2014) \\
\hline 145 & Adeona & $2.78 \times 10^{18}$ & $1.79 \times 10^{18}$ & DEFL & Goffin (2014) \\
\hline 145 & Adeona & $3.24 \times 10^{17}$ & $4.80 \times 10^{17}$ & EPHEM & Fienga (2018, priv. comm.) \\
\hline 146 & Lucina & $1.18 \times 10^{17}$ & $1.77 \times 10^{17}$ & EPHEM & Fienga (2018, priv. comm.) \\
\hline 156 & Xanthippe & $6.49 \times 10^{18}$ & $2.62 \times 10^{18}$ & EPHEM & Fienga et al. (2013) \\
\hline 156 & Xanthippe & $6.72 \times 10^{17}$ & $9.00 \times 10^{17}$ & EPHEM & Fienga (2018, priv. comm.) \\
\hline 159 & Aemilia & $4.18 \times 10^{18}$ & $1.79 \times 10^{18}$ & DEFL & Goffin (2014) \\
\hline 159 & Aemilia & $5.72 \times 10^{18}$ & $7.59 \times 10^{18}$ & EPHEM & Fienga (2018, priv. comm.) \\
\hline 162 & Laurentia & $3.36 \times 10^{17}$ & $5.01 \times 10^{17}$ & EPHEM & Fienga (2018, priv. comm.) \\
\hline 163 & Erigone & $5.79 \times 10^{17}$ & $7.29 \times 10^{17}$ & EPHEM & Fienga (2018, priv. comm.) \\
\hline 168 & Sibylla & $6.02 \times 10^{18}$ & $2.35 \times 10^{19}$ & DEFL & Zielenbach (2011) \\
\hline 168 & Sibylla & $2.38 \times 10^{18}$ & $1.72 \times 10^{19}$ & DEFL & Zielenbach (2011) \\
\hline 168 & Sibylla & $6.33 \times 10^{18}$ & $7.32 \times 10^{18}$ & EPHEM & Fienga (2018, priv. comm.) \\
\hline 176 & Iduna & $3.36 \times 10^{17}$ & $5.04 \times 10^{17}$ & EPHEM & Fienga (2018, priv. comm.) \\
\hline 187 & Lamberta & $1.57 \times 10^{18}$ & $4.71 \times 10^{17}$ & EPHEM & Folkner et al. (2009) \\
\hline 187 & Lamberta & $5.69 \times 10^{18}$ & $2.35 \times 10^{18}$ & EPHEM & Fienga et al. (2014) \\
\hline 187 & Lamberta & $2.19 \times 10^{18}$ & $2.39 \times 10^{18}$ & DEFL & Goffin (2014) \\
\hline 187 & Lamberta & $2.87 \times 10^{17}$ & $4.14 \times 10^{17}$ & EPHEM & Viswanathan et al. (2017) \\
\hline 187 & Lamberta & $4.93 \times 10^{17}$ & $6.81 \times 10^{17}$ & EPHEM & Fienga (2018, priv. comm.) \\
\hline 195 & Eurykleia & $1.44 \times 10^{17}$ & $2.16 \times 10^{17}$ & EPHEM & Fienga (2018, priv. comm.) \\
\hline 200 & Dynamene & $1.14 \times 10^{18}$ & $4.17 \times 10^{17}$ & EPHEM & Fienga et al. (2013) \\
\hline 200 & Dynamene & $3.98 \times 10^{18}$ & $1.79 \times 10^{18}$ & DEFL & Goffin (2014) \\
\hline 205 & Martha & $1.19 \times 10^{17}$ & $1.78 \times 10^{17}$ & EPHEM & Fienga (2018, priv. comm.) \\
\hline 211 & Isolda & $1.99 \times 10^{18}$ & $6.84 \times 10^{18}$ & DEFL & Zielenbach (2011) \\
\hline 211 & Isolda & $2.67 \times 10^{18}$ & $5.34 \times 10^{18}$ & DEFL & Zielenbach (2011) \\
\hline 211 & Isolda & $2.41 \times 10^{18}$ & $5.34 \times 10^{18}$ & DEFL & Zielenbach (2011) \\
\hline 211 & Isolda & $4.16 \times 10^{18}$ & $7.98 \times 10^{18}$ & DEFL & Zielenbach (2011) \\
\hline 211 & Isolda & $7.83 \times 10^{18}$ & $9.39 \times 10^{18}$ & EPHEM & Fienga et al. (2011) \\
\hline 211 & Isolda & $2.98 \times 10^{18}$ & $1.79 \times 10^{18}$ & DEFL & Goffin (2014) \\
\hline 211 & Isolda & $6.36 \times 10^{18}$ & $6.81 \times 10^{18}$ & EPHEM & Viswanathan et al. (2017) \\
\hline 211 & Isolda & $5.40 \times 10^{18}$ & $6.12 \times 10^{18}$ & EPHEM & Fienga (2018, priv. comm.) \\
\hline 238 & Hypatia & $4.86 \times 10^{18}$ & $1.25 \times 10^{19}$ & DEFL & Zielenbach (2011) \\
\hline
\end{tabular}


Table D.3. continued.

\begin{tabular}{|c|c|c|c|c|c|}
\hline$\#$ & Name & $\begin{array}{c}\mathcal{M} \\
(\mathrm{kg})\end{array}$ & $\begin{array}{l}\delta \mathcal{M} \\
(\mathrm{kg})\end{array}$ & Method & Reference \\
\hline 238 & Hypatia & $5.44 \times 10^{18}$ & $9.81 \times 10^{18}$ & DEFL & Zielenbach (2011) \\
\hline 238 & Hypatia & $6.20 \times 10^{18}$ & $9.72 \times 10^{18}$ & DEFL & Zielenbach (2011) \\
\hline 238 & Hypatia & $8.63 \times 10^{17}$ & $2.18 \times 10^{19}$ & DEFL & Zielenbach (2011) \\
\hline 238 & Hypatia & $2.98 \times 10^{18}$ & $1.79 \times 10^{18}$ & DEFL & Goffin (2014) \\
\hline 238 & Hypatia & $4.79 \times 10^{18}$ & $5.67 \times 10^{18}$ & EPHEM & Fienga (2018, priv. comm.) \\
\hline 266 & Aline & $4.23 \times 10^{18}$ & $6.33 \times 10^{18}$ & EPHEM & Viswanathan et al. (2017) \\
\hline 266 & Aline & $1.43 \times 10^{18}$ & $2.08 \times 10^{18}$ & EPHEM & Fienga (2018, priv. comm.) \\
\hline 303 & Josephina & $1.31 \times 10^{17}$ & $1.97 \times 10^{17}$ & EPHEM & Fienga (2018, priv. comm.) \\
\hline 345 & Tercidina & $2.68 \times 10^{18}$ & $3.54 \times 10^{18}$ & EPHEM & Fienga et al. (2011) \\
\hline 345 & Tercidina & $3.53 \times 10^{18}$ & $3.48 \times 10^{18}$ & EPHEM & Viswanathan et al. (2017) \\
\hline 345 & Tercidina & $1.17 \times 10^{18}$ & $1.53 \times 10^{18}$ & EPHEM & Fienga (2018, priv. comm.) \\
\hline 350 & Ornamenta & $2.59 \times 10^{17}$ & $3.87 \times 10^{17}$ & EPHEM & Fienga (2018, priv. comm.) \\
\hline 356 & Liguria & $7.83 \times 10^{18}$ & $4.50 \times 10^{18}$ & ЕРHEM & Fienga et al. (2011) \\
\hline 356 & Liguria & $8.30 \times 10^{18}$ & $3.45 \times 10^{18}$ & EPHEM & Fienga et al. (2013) \\
\hline 356 & Liguria & $2.78 \times 10^{18}$ & $1.19 \times 10^{18}$ & DEFL & Goffin (2014) \\
\hline 356 & Liguria & $3.98 \times 10^{18}$ & $2.11 \times 10^{18}$ & EPHEM & Viswanathan et al. (2017) \\
\hline 356 & Liguria & $9.05 \times 10^{17}$ & $1.22 \times 10^{18}$ & EPHEM & Fienga (2018, priv. comm.) \\
\hline 358 & Apollonia & $1.60 \times 10^{17}$ & $2.40 \times 10^{17}$ & EPHEM & Fienga (2018, priv. comm.) \\
\hline 362 & Havnia & $1.67 \times 10^{17}$ & $2.51 \times 10^{17}$ & EPHEM & Fienga (2018, priv. comm.) \\
\hline 366 & Vincentina & $1.00 \times 10^{17}$ & $1.50 \times 10^{17}$ & EPHEM & Fienga (2018, priv. comm.) \\
\hline 373 & Melusina & $3.43 \times 10^{17}$ & $5.16 \times 10^{17}$ & EPHEM & Fienga (2018, priv. comm.) \\
\hline 377 & Campania & $1.52 \times 10^{17}$ & $2.28 \times 10^{17}$ & EPHEM & Fienga (2018, priv. comm.) \\
\hline 404 & Arsinoe & $1.25 \times 10^{18}$ & $3.51 \times 10^{18}$ & EPHEM & Fienga et al. (2013) \\
\hline 404 & Arsinoe & $7.87 \times 10^{17}$ & $9.84 \times 10^{17}$ & EPHEM & Fienga (2018, priv. comm.) \\
\hline 405 & Thia & $1.38 \times 10^{18}$ & $4.14 \times 10^{17}$ & EPHEM & Folkner et al. (2009) \\
\hline 405 & Thia & $2.74 \times 10^{18}$ & $1.97 \times 10^{18}$ & EPHEM & Fienga et al. (2013) \\
\hline 405 & Thia & $6.38 \times 10^{18}$ & $1.12 \times 10^{18}$ & EPHEM & Fienga et al. (2014) \\
\hline 405 & Thia & $2.19 \times 10^{18}$ & $2.98 \times 10^{18}$ & DEFL & Goffin (2014) \\
\hline 405 & Thia & $3.13 \times 10^{18}$ & $1.39 \times 10^{18}$ & EPHEM & Viswanathan et al. (2017) \\
\hline 405 & Thia & $2.80 \times 10^{18}$ & $1.28 \times 10^{18}$ & EPHEM & Fienga (2018, priv. comm.) \\
\hline 407 & Arachne & $1.26 \times 10^{17}$ & $1.88 \times 10^{17}$ & EPHEM & Fienga (2018, priv. comm.) \\
\hline 410 & Chloris & $6.11 \times 10^{18}$ & $2.76 \times 10^{18}$ & EPHEM & Fienga et al. (2011) \\
\hline 410 & Chloris & $6.91 \times 10^{18}$ & $3.93 \times 10^{18}$ & ЕРНЕM & Fienga et al. (2013) \\
\hline 410 & Chloris & $5.33 \times 10^{18}$ & $2.58 \times 10^{18}$ & EPHEM & Viswanathan et al. (2017) \\
\hline 410 & Chloris & $1.89 \times 10^{18}$ & $1.79 \times 10^{18}$ & EPHEM & Fienga (2018, priv. comm.) \\
\hline 442 & Eichsfeldia & $2.50 \times 10^{17}$ & $3.69 \times 10^{17}$ & EPHEM & Fienga (2018, priv. comm.) \\
\hline 445 & Edna & $4.18 \times 10^{18}$ & $3.57 \times 10^{18}$ & DEFL & Goffin (2014) \\
\hline 445 & Edna & $1.24 \times 10^{18}$ & $1.83 \times 10^{18}$ & EPHEM & Fienga (2018, priv. comm.) \\
\hline 481 & Emita & $1.31 \times 10^{18}$ & $1.87 \times 10^{18}$ & EPHEM & Fienga (2018, priv. comm.) \\
\hline 488 & Kreusa & $2.46 \times 10^{18}$ & $7.38 \times 10^{17}$ & EPHEM & Folkner et al. (2009) \\
\hline 488 & Kreusa & $1.60 \times 10^{19}$ & $1.85 \times 10^{19}$ & DEFL & Zielenbach (2011) \\
\hline 488 & Kreusa & $2.29 \times 10^{18}$ & $1.99 \times 10^{18}$ & DEFL & Zielenbach (2011) \\
\hline 488 & Kreusa & $2.19 \times 10^{18}$ & $2.00 \times 10^{18}$ & DEFL & Zielenbach (2011) \\
\hline 488 & Kreusa & $8.25 \times 10^{17}$ & $4.11 \times 10^{19}$ & DEFL & Zielenbach (2011) \\
\hline 488 & Kreusa & $9.77 \times 10^{18}$ & $8.10 \times 10^{18}$ & EPHEM & Fienga et al. (2011) \\
\hline 488 & Kreusa & $1.03 \times 10^{19}$ & $1.08 \times 10^{19}$ & EPHEM & Fienga et al. (2013) \\
\hline 488 & Kreusa & $8.58 \times 10^{17}$ & $1.01 \times 10^{19}$ & EPHEM & Fienga et al. (2014) \\
\hline 488 & Kreusa & $7.36 \times 10^{18}$ & $2.98 \times 10^{18}$ & DEFL & Goffin (2014) \\
\hline 488 & Kreusa & $4.65 \times 10^{18}$ & $5.58 \times 10^{18}$ & EPHEM & Viswanathan et al. (2017) \\
\hline 488 & Kreusa & $4.63 \times 10^{18}$ & $5.28 \times 10^{18}$ & EPHEM & Fienga (2018, priv. comm.) \\
\hline 490 & Veritas & $9.32 \times 10^{18}$ & $1.31 \times 10^{19}$ & DEFL & Zielenbach (2011) \\
\hline 490 & Veritas & $4.48 \times 10^{18}$ & $6.33 \times 10^{18}$ & DEFL & Zielenbach (2011) \\
\hline 490 & Veritas & $4.59 \times 10^{18}$ & $6.30 \times 10^{18}$ & DEFL & Zielenbach (2011) \\
\hline 490 & Veritas & $9.51 \times 10^{18}$ & $1.69 \times 10^{19}$ & DEFL & Zielenbach (2011) \\
\hline 490 & Veritas & $2.02 \times 10^{18}$ & $2.96 \times 10^{18}$ & EPHEM & Fienga (2018, priv. comm.) \\
\hline
\end{tabular}


Table D.3. continued.

\begin{tabular}{|c|c|c|c|c|c|}
\hline$\#$ & Name & $\underset{(\mathrm{kg})}{\mathcal{M}}$ & $\begin{array}{l}\delta \mathcal{M} \\
(\mathrm{kg})\end{array}$ & Method & Reference \\
\hline 503 & Evelyn & $8.28 \times 10^{17}$ & $1.15 \times 10^{18}$ & EPHEM & Fienga (2018, priv. comm.) \\
\hline 521 & Brixia & $7.09 \times 10^{17}$ & $1.01 \times 10^{18}$ & EPHEM & Viswanathan et al. (2017) \\
\hline 521 & Brixia & $3.37 \times 10^{17}$ & $4.98 \times 10^{17}$ & EPHEM & Fienga (2018, priv. comm.) \\
\hline 554 & Peraga & $6.59 \times 10^{17}$ & $1.98 \times 10^{17}$ & EPHEM & Folkner et al. (2009) \\
\hline 554 & Peraga & $7.95 \times 10^{17}$ & $5.97 \times 10^{17}$ & DEFL & Goffin (2014) \\
\hline 554 & Peraga & $2.34 \times 10^{17}$ & $3.78 \times 10^{17}$ & EPHEM & Fienga (2018, priv. comm.) \\
\hline 602 & Marianna & $3.20 \times 10^{18}$ & $4.08 \times 10^{18}$ & EPHEM & Fienga (2018, priv. comm.) \\
\hline 654 & Zelinda & $1.35 \times 10^{18}$ & $4.05 \times 10^{17}$ & EPHEM & Folkner et al. (2009) \\
\hline 654 & Zelinda & $6.76 \times 10^{18}$ & $2.98 \times 10^{18}$ & DEFL & Goffin (2014) \\
\hline 654 & Zelinda & $7.41 \times 10^{17}$ & $7.83 \times 10^{17}$ & EPHEM & Fienga (2018, priv. comm.) \\
\hline 694 & Ekard & $1.20 \times 10^{17}$ & $1.78 \times 10^{17}$ & EPHEM & Fienga (2018, priv. comm.) \\
\hline 735 & Marghanna & $7.23 \times 10^{17}$ & $8.94 \times 10^{17}$ & EPHEM & Fienga (2018, priv. comm.) \\
\hline 751 & Faina & $3.27 \times 10^{18}$ & $1.75 \times 10^{18}$ & EPHEM & Fienga et al. (2011) \\
\hline 751 & Faina & $4.53 \times 10^{18}$ & $3.30 \times 10^{18}$ & EPHEM & Viswanathan et al. (2017) \\
\hline 751 & Faina & $4.13 \times 10^{18}$ & $3.03 \times 10^{18}$ & EPHEM & Fienga (2018, priv. comm.) \\
\hline 776 & Berbericia & $5.46 \times 10^{18}$ & $1.41 \times 10^{19}$ & DEFL & Zielenbach (2011) \\
\hline 776 & Berbericia & $2.39 \times 10^{16}$ & $9.78 \times 10^{18}$ & DEFL & Zielenbach (2011) \\
\hline 776 & Berbericia & $3.08 \times 10^{17}$ & $9.72 \times 10^{18}$ & DEFL & Zielenbach (2011) \\
\hline 776 & Berbericia & $6.28 \times 10^{18}$ & $2.19 \times 10^{19}$ & DEFL & Zielenbach (2011) \\
\hline 776 & Berbericia & $8.11 \times 10^{18}$ & $6.69 \times 10^{18}$ & EPHEM & Fienga (2018, priv. comm.) \\
\hline 788 & Hohensteina & $1.85 \times 10^{17}$ & $2.77 \times 10^{17}$ & EPHEM & Fienga (2018, priv. comm.) \\
\hline 791 & Ani & $1.47 \times 10^{17}$ & $2.21 \times 10^{17}$ & EPHEM & Fienga (2018, priv. comm.) \\
\hline 914 & Palisana & $1.97 \times 10^{18}$ & $2.16 \times 10^{18}$ & EPHEM & Viswanathan et al. (2017) \\
\hline 914 & Palisana & $4.89 \times 10^{17}$ & $6.87 \times 10^{17}$ & EPHEM & Fienga (2018, priv. comm.) \\
\hline 1467 & Mashona & $2.04 \times 10^{17}$ & $3.06 \times 10^{17}$ & EPHEM & Fienga (2018, priv. comm.) \\
\hline
\end{tabular}

